\title{
Topological invariants from nonrestricted quantum groups
}

\author{
NATHAN GEER \\ BERTRAND PATUREAU-MirAND
}

\begin{abstract}
We introduce the notion of a relative spherical category. We prove that such a category gives rise to the generalized Kashaev and Turaev-Viro-type 3-manifold invariants defined in [12] and [17], respectively. In this case we show that these invariants are equal and extend to what we call a relative homotopy quantum field theory, which is a branch of the topological quantum field theory founded by E Witten and M Atiyah. Our main examples of relative spherical categories are the categories of finite-dimensional weight modules over nonrestricted quantum groups considered by C De Concini, V Kac, C Procesi, N Reshetikhin and M Rosso. These categories are not semisimple and have an infinite number of nonisomorphic irreducible modules all having vanishing quantum dimensions. We also show that these categories have associated ribbon categories which gives rise to renormalized link invariants. In the case of $\mathfrak{s l}_{2}$ these link invariants are the Alexander-type multivariable invariants defined by Y Akutsu, T Deguchi and T Ohtsuki [1].
\end{abstract}

17B37, 57M25, 57M27

\section{Introduction}

A principal feature of quantum topology is its interplay between tensor categories and low-dimensional topology. The fundamental example of such interplay is the modular category formed from finite-dimensional representation of the restricted quantum group $U_{q}\left(\mathfrak{s l}_{2}\right)$ at a root of unity and the corresponding Reshetikhin-Turaev and Turaev-Viro 3-manifold invariants. Loosely speaking, this modular category is the category of all representations over $U_{q}\left(\mathfrak{s l}_{2}\right)$ quotiented by the ideal of representation with zero quantum dimension. This category is semisimple with a finite number of isomorphism classes of irreducible representations all having nonvanishing quantum dimensions. Generalizations to other semisimple categories were done by several authors including H Andersen, V Turaev, H Wenzl, J Barrett, B Westbury, A Ocneanu, S Gelfand, D Kazhdan and others. 
Less progress has been made in categories which have simple objects with vanishing quantum dimensions. This is true even though the Volume Conjecture naturally arises in such a setting (see Murakami and Murakami [23]). In the case of $\mathfrak{s l}_{2}, 3$-manifold invariants arising from representations with zero quantum dimensions have been studied by two approaches: (1) R Kashaev's geometric and physical construction in [20; 21], which was extended by S Baseilhac and R Benedetti [2] and (2) the topological and algebraic Turaev-Viro-type invariants of N Geer, B Patureau-Mirand and V Turaev [17]. In [12], Geer, Kashaev and Turaev used the notion of a $\widehat{\Psi}$-system to generalize Kashaev's construction to a categorical setting.

This paper has five main results: First, we show that the nonrestricted quantum groups associated to simple Lie algebras considered by C De Concini and V Kac, in [8], admit Turaev-Viro-type invariants arising from representations with zero quantum dimensions. Second, we prove that such quantum groups also lead to $\hat{\Psi}$-systems and so from [12] admit generalized Kashaev invariants. Third, we show that in this situation the generalized Kashaev and TV-type invariants are equal. Fourth, we extend the above mentioned TV-type invariants to a kind of homotopy quantum field theory. Finally, we prove that certain representations (with zero quantum dimensions) over nonrestricted quantum groups give rise to renormalized Reshetikhin-Turaev link invariants. All of these results are proved in a general categorical language which also contains as examples the usual modular categories formed from representations of restricted quantum groups.

This paper has two major components. The aim of first component is to introduce and study "relative spherical categories". These categories are generalizations of the usual modular categories associated to restricted quantum groups (see Theorem 4). However, in general they are not necessarily semisimple (only "generically" semisimple) and can have an infinite number of nonisomorphic simple objects all having vanishing quantum dimensions. In Section 3.4, we will show that a relative spherical category $\mathcal{C}$ leads to a Turaev-Viro-type invariant $T V$ of triples (a closed oriented 3-manifold $M$, a link in $M$, a conjugacy class of homomorphisms $\pi_{1}(M) \rightarrow \mathcal{G}$ ), where $\mathcal{G}$ is a group which is part of the data of the category. Then in Section 3.6 we will also show that $\mathcal{C}$ gives rise to a $\hat{\Psi}$-system. Thus, the construction of [12] gives a Kashaev-type invariant $\mathrm{K}$ and in Section 3.6 we prove that $T V=\mathrm{K}$. The final piece of the first component of this paper is to show that $T V$ can be extended to what we call a "relative homotopy quantum field theory" (relative HQFT); see Section 4. This relative HQFT is similar in nature to Turaev's [28] homotopy quantum field theory which is a branch of topological quantum field theory founded by E Witten and M Atiyah. However, we have cobordism with graphs inside. Our relative HQFT is also similar to the quantum hyperbolic field theory coming from the Borel subalgebra of quantum $\mathfrak{s l}_{2}$ defined in Baseilhac and 
Benedetti's [3]. One can hope to make a precise relationship with these different field theories.

The aim of the second component of this paper is to construct invariants of links and 3-manifolds from representations over nonrestricted quantum groups which are only "generically" semisimple and have vanishing quantum dimensions. C De Concini, V Kac, C Procesi, N Reshetikhin and M Rosso have established a deep body of work pertaining to quantum groups and their representation theory, at odd ordered roots of unity; see the series of papers [8], [9], [10] and [11]. In Section 5 we recall some of their results and prove that the representations studied in these papers give rise to three topological invariants: $T V, \mathrm{~K}$ and a Reshetikhin-Turaev- or Akutsu-Deguchi-Ohtsukitype link invariant (see Theorem 8). The representations considered here have zero quantum dimensions and so the usual associated topological invariants are trivial. To overcome this difficultly we show that these representations are part of an ambidextrous pair with modified dimension $d$. This modified dimension is used to renormalize the usual link invariants and the quantum $6 j$-symbols. Let us be more precise. For an odd ordered root of unity, let $\mathcal{U}$ and $\mathcal{U}^{H}$ be the quantum groups associated to a fixed simple Lie algebra, described in Section 5.1. We prove that the finite-dimensional weight modules over $\mathcal{U}^{H}$ contain a ribbon category which admits an ambidextrous pair. Combining this with a direct calculation of the open Hopf link we prove that Geer, Patureau-Mirand and Turaev's results [16] can be applied and lead to link invariants (see Theorem 8 and Proposition 45). Then incorporating all the results of Section 5 we show that the finite-dimensional weight modules over $\mathcal{U}$ form a relative spherical category and so the results described in the previous paragraph can be applied. In particular, this shows that the category of finite-dimensional weight modules over $\mathcal{U}$ has several $\widehat{\Psi}$-systems (giving a positive answer to [12, Conjecture 42] for $\mathcal{U}$ ) and proves that the corresponding generalized Kashaev and Turaev-Viro-type 3-manifold invariants are equal.

The work of N Geer was partially supported by the NSF grants DMS-0706725 and DMS-1007197. B Patureau-Mirand thanks Utah State University and the members of its mathematics department for their generous hospitality.

\section{Relative $\mathcal{G}$-spherical categories and $\widehat{\Psi}$-systems}

In this section we give the basic categorical definitions used in this paper. In Section 1.1 recall some fairly well-known notations involving tensor categories. In Sections 1.2 and 1.4 we discuss two less known notions: ambidextrous traces and $\hat{\Psi}$-systems. These two notions will be used to construct nontrivial invariants when quantum dimensions 
are zero. In Section 1.3 we give the notion of relative $\mathcal{G}$-spherical categories. Such categories are used to deal with the fact that our categories are not necessarily semisimple but only "generically" semisimple.

\subsection{Linear tensor categories}

A tensor category $\mathcal{C}$ is a category equipped with a covariant bifunctor $\otimes: \mathcal{C} \times \mathcal{C} \rightarrow \mathcal{C}$ called the tensor product, an associativity constraint, a unit object $\mathbb{I}$, and left and right unit constraints such that the Triangle and Pentagon Axioms hold. Let $\mathbb{k}$ be an integral domain. A tensor category $\mathcal{C}$ is said to be $\mathbb{k}$-linear if its hom-sets are $\mathbb{k}$-modules, the composition and tensor product of morphisms are $\mathbb{k}$-bilinear, and $\operatorname{End}_{\mathcal{C}}(\mathbb{I})$ is a free $\mathbb{k}$-module of rank one. Then we identify $\mathbb{k}=\operatorname{End}_{\mathcal{C}}(\mathbb{I})$, via the ring isomorphism $\mathbb{k} \rightarrow \operatorname{End}_{\mathcal{C}}(\mathbb{I}), k \mapsto k \operatorname{Id}_{\mathbb{I}}$ and call $\mathbb{k}$ the ground ring of $\mathcal{C}$. Let $\mathbb{k}^{\times}$be the set of invertible elements of $\mathbb{k}$. An object $V$ of $\mathcal{C}$ is simple if $\operatorname{End}_{\mathcal{C}}(V)=\mathbb{k} \operatorname{Id}_{V}$. An object which is a direct sum of simple objects is called semisimple. For any simple object $V$ and $f \in \operatorname{End}(V)$, there is a unique $x \in \mathbb{k}$ such that $f=x \operatorname{Id}_{V}$. This $x$ is denoted $\langle f\rangle$.

If $\mathcal{C}$ is an $\mathbb{k}$-linear category and $\left\{V_{i}\right\}_{i \in I}$ is a set of simple objects numerated by a set $I$, for $i, j, k \in I$ we consider the following $\mathbb{k}$-modules:

$$
\begin{aligned}
H^{i j k} & =\operatorname{Hom}_{\mathcal{C}}\left(\mathbb{I}, V_{i} \otimes V_{j} \otimes V_{k}\right) \\
H_{k}^{i j} & =\operatorname{Hom}_{\mathcal{C}}\left(V_{k}, V_{i} \otimes V_{j}\right) \\
H_{i j}^{k} & =\operatorname{Hom}_{\mathcal{C}}\left(V_{i} \otimes V_{j}, V_{k}\right)
\end{aligned}
$$

A pivotal category is a tensor category with duality morphisms $b_{V}: \mathbb{I} \rightarrow V \otimes V^{*}$, $d_{V}: V^{*} \otimes V \rightarrow \mathbb{I}, b_{V}^{\prime}: \mathbb{I} \rightarrow V^{*} \otimes V$ and $d_{V}^{\prime}: V \otimes V^{*} \rightarrow \mathbb{I}$ which satisfy compatibility conditions (see for example [17]).

Let $\mathcal{C}$ be a pivotal category. A morphism $f: V_{1} \otimes \cdots \otimes V_{n} \rightarrow W_{1} \otimes \cdots \otimes W_{m}$ in $\mathcal{C}$ can be represented by a box and arrows:

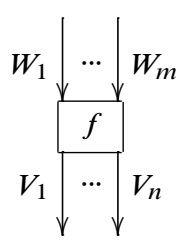

Boxes as above are called coupons. By a $\mathcal{C}$-colored ribbon graph in an oriented surface $\Sigma$, we mean a graph embedded in $\Sigma$ whose edges are colored by objects of $\mathcal{C}$ and 
whose vertices lying in Int $\Sigma=\Sigma \backslash \partial \Sigma$ are thickened to coupons colored by morphisms of $\mathcal{C}$. Let $\mathrm{Gr}_{\mathcal{C}}$ be the category of $\mathcal{C}$-colored ribbon graphs in $\mathbb{R} \times[0,1]$ and $\mathrm{G} \operatorname{Gr}_{\mathcal{C}} \rightarrow \mathcal{C}$ be the Reshetikhin-Turaev $\mathbb{k}$-linear functor preserving the duality morphism of $\mathcal{C}$ (see [17]).

A ribbon category is a pivotal category with a braiding $c_{V, W}: V \otimes W \rightarrow W \otimes V$ and twist $\theta_{V}: V \rightarrow V$ which are compatible with the pivotal structure (see [27, Chapter 1]). If $\mathcal{C}$ is a ribbon category, the Reshetikhin-Turaev functor $\mathrm{G}$ extend as $\mathrm{G}: \mathrm{Rib}_{\mathcal{C}} \rightarrow \mathcal{C}$, where $\mathrm{Rib}_{\mathcal{C}}$ is the category of $\mathcal{C}$-colored ribbon graphs in $\mathbb{R}^{2} \times[0,1]$.

\subsection{Ambidextrous pairs and traces}

We recall the process of renormalizing colored ribbon graphs first used in $[14 ; 15]$ then generalized using the notion of ambidextrous entities in $[13 ; 16 ; 17 ; 18]$.

Let $\mathcal{C}$ be a $\mathbb{k}$-linear pivotal (resp. ribbon) category and let $T \subset S^{2}$ (resp. $T \subset S^{3}$ ) be a closed $\mathcal{C}$-colored ribbon graph. Let $e$ be an edge of $T$ colored with a simple object $V$ of $\mathcal{C}$. Cutting $T$ at a point of $e$, we obtain a $\mathcal{C}$-colored ribbon graph $T_{V}$ in $\mathbb{R} \times[0,1]$ (resp. in $\mathbb{R}^{2} \times[0,1]$ ), where $\mathrm{G}\left(T_{V}\right) \in \operatorname{End}(V)=\mathbb{k} \operatorname{Id}_{V}$. We call $T_{V}$ a cutting presentation of $T$ and let $\left\langle T_{V}\right\rangle \in \mathbb{k}$ denote the isotopy invariant of $T_{V}$ defined from the equality $\mathrm{G}\left(T_{V}\right)=\left\langle T_{V}\right\rangle \mathrm{Id}_{V}$.

Let $\mathrm{A}$ be a class of simple objects of $\mathcal{C}$ and $\mathrm{d}: \mathrm{A} \rightarrow \mathbb{k}^{\times}$be a mapping such that $\mathrm{d}(V)=\mathrm{d}\left(V^{*}\right)$ and $\mathrm{d}(V)=\mathrm{d}\left(V^{\prime}\right)$ if $V$ is isomorphic to $V^{\prime}$. Let $T$ be a closed $\mathcal{C}$-colored ribbon graph which admit two cutting presentations $T_{V}$ and $T_{V^{\prime}}$ with both $V$ and $V^{\prime}$ in A. Then we consider if the following equality holds:

$$
\mathrm{d}(V)\left\langle T_{V}\right\rangle=\mathrm{d}\left(V^{\prime}\right)\left\langle T_{V}^{\prime}\right\rangle .
$$

First, suppose that $\mathcal{C}$ is a $\mathbb{k}$-linear ribbon category and let $\mathcal{L}_{\mathrm{A}}$ be the class of $\mathcal{C}$-colored ribbon graphs in $S^{3}$ with at least one edge colored by an element of $A$. Suppose that Equation (1) holds for all $T \in \mathcal{L}_{\mathrm{A}}$. Then we say that $(\mathrm{A}, \mathrm{d})$ is an ambidextrous pair or ambi for short.

On the other hand, suppose that $\mathcal{C}$ is a $\mathbb{k}$-linear pivotal category. In [17] a smaller class of graphs is considered: A ribbon graph is trivalent if all its coupons are adjacent to 3 half-edges. Let $\mathcal{T}_{\mathrm{A}}$ be the class of $\mathcal{C}$-colored connected trivalent ribbon graphs in $S^{2}$ such that the colors of all edges belong to A. Suppose that Equation (1) holds for all $T \in \mathcal{T}_{\mathrm{A}}$. Then we say that $(\mathrm{A}, \mathrm{d})$ is a trivalent-ambidextrous pair or $t$-ambi for short. 
If $(A, d)$ is ambi (resp. t-ambi) we define a function $G^{\prime}: \mathcal{L}_{A} \rightarrow \mathbb{k}\left(\right.$ resp. $\left.G^{\prime}: \mathcal{T}_{A} \rightarrow \mathbb{k}\right)$ by

$$
\mathrm{G}^{\prime}(T)=\mathrm{d}(V)\left\langle T_{V}\right\rangle,
$$

where $T_{V}$ is any cutting presentation of $T$ with $V \in \mathrm{A}$. The above definitions imply that $G^{\prime}$ is well defined. With Equation (2) in mind, we call $d$ a modified quantum dimension.

Let Proj be the full subcategory of $\mathcal{C}$ consisting of projective objects. A trace on Proj is a family of $\mathbb{k}$-linear functions $\mathrm{t}=\left\{\mathrm{t}_{V}: \operatorname{End}_{\mathcal{C}}(V) \rightarrow \mathbb{k}\right\} V \in$ Proj which is suitably compatible with the tensor product and composition of morphisms (see [13; 18]). Let $A_{\text {Proj }}$ be the set of simple projective objects of $\mathcal{C}$. A trace $t=\left\{t_{V}\right\}_{V \in \text { Proj }}$ defines a function d: $A_{\text {Proj }} \rightarrow \mathbb{k}^{\times}$given by $\mathrm{d}(V)=\mathrm{t}_{V}\left(\operatorname{Id}_{V}\right)$. If $\mathrm{d}(V)=\mathrm{d}\left(V^{*}\right)$ for all $V \in$ Proj then $\left(A_{\text {Proj }}, d\right)$ is $(t-)$ ambi and the corresponding isotopy invariant $G^{\prime}$ is determined by the trace:

$$
\mathrm{G}^{\prime}(T)=\mathrm{d}(V)\left\langle T_{V}\right\rangle=\mathrm{t}_{V}\left(\left\langle T_{V}\right\rangle \mathrm{Id}_{V}\right)=\mathrm{t}_{V}\left(T_{V}\right),
$$

where $T_{V}$ is any cutting presentation of $T$ (see $\left.[13 ; 18]\right)$.

\subsection{Relative $\mathcal{G}$-spherical categories}

We now fix a group $\mathcal{G}$.

Definition 1 A pivotal category is $\mathcal{G}$-graded if for each $g \in \mathcal{G}$ we have a nonempty full subcategory $\mathcal{C}_{g}$ of $\mathcal{C}$ such that:

(i) $\mathcal{C}=\bigoplus_{g \in \mathcal{G}} \mathcal{C}_{g}$.

(ii) If $V \in \mathcal{C}_{g}$, then $V^{*} \in \mathcal{C}_{g^{-1}}$.

(iii) If $V \in \mathcal{C}_{g}, W \in \mathcal{C}$ and $V$ is isomorphic to $W$, then $W \in \mathcal{C}_{g}$.

(iv) If $V \in \mathcal{C}_{g}, V^{\prime} \in \mathcal{C}_{g^{\prime}}$ then $V \otimes V^{\prime} \in \mathcal{C}_{g g^{\prime}}$.

(v) If $V \in \mathcal{C}_{g}, V^{\prime} \in \mathcal{C}_{g^{\prime}}$ and $\operatorname{Hom}_{\mathcal{C}}\left(V, V^{\prime}\right) \neq 0$, then $g=g^{\prime}$.

Let $\mathcal{X} \subset \mathcal{G}$ be a subset with the following properties:

(i) $\mathcal{X}$ is symmetric: $\mathcal{X}^{-1}=\mathcal{X}$.

(ii) $\mathcal{G}$ can not be covered by a finite number of translated copies of $\mathcal{X}$, in other words, for any $g_{1}, \ldots, g_{n} \in \mathcal{G}$, we have $\bigcup_{i=1}^{n}\left(g_{i} \mathcal{X}\right) \neq \mathcal{G}$.

Let us write $\mathcal{G}^{\prime}=\mathcal{G} \backslash \mathcal{X}$. 
Definition 2 Let $\mathcal{C}$ be a $\mathcal{G}$-graded $\mathbb{k}$-linear pivotal category. Let $\mathrm{A}$ be the class of all simple objects in $\bigcup_{g \in \mathcal{G}^{\prime}} \mathcal{C}_{g}$. We say that $\mathcal{C}$ is $(\mathcal{X}, \mathrm{d})$-relative $\mathcal{G}$-spherical if

(i) for each $g \in \mathcal{G}^{\prime}, \mathcal{C}_{g}$ is semisimple (ie all objects of $\mathcal{C}_{g}$ are semisimple) with finitely many isomorphism classes of simple objects,

(ii) there exists a map $d: A \rightarrow \mathbb{k}^{\times}$such that $(A, d)$ is a t-ambi pair,

(iii) there exists a map $\mathrm{b}: \mathrm{A} \rightarrow \mathbb{k}$ such that $\mathrm{b}(V)=\mathrm{b}\left(V^{*}\right), \mathrm{b}(V)=\mathrm{b}\left(V^{\prime}\right)$ for any isomorphic objects $V, V^{\prime} \in \mathrm{A}$ and for any $g_{1}, g_{2}, g_{1} g_{2} \in \mathcal{G} \backslash \mathcal{X}$ and $V \in \mathcal{G}_{g_{1} g_{2}}$ we have

$$
\mathrm{b}(V)=\sum_{\substack{V_{1} \in \operatorname{irr}\left(\mathcal{C}_{g_{1}}\right) \\ V_{2} \in \operatorname{irr}\left(\mathcal{C}_{g_{2}}\right)}} \mathrm{b}\left(V_{1}\right) \mathrm{b}\left(V_{2}\right) \operatorname{dim}_{\mathbb{k}}\left(\operatorname{Hom}_{\mathcal{C}}\left(V, V_{1} \otimes V_{2}\right)\right),
$$

where $\operatorname{irr}\left(\mathcal{C}_{g_{i}}\right)$ denotes a representing set of the isomorphism classes of simple objects of $\mathcal{D}_{g_{i}}$.

If $\mathcal{C}$ is a category with the above data, for brevity we say $\mathcal{C}$ is a relative $\mathcal{G}$-spherical category.

The map $b$ always exists when $\mathbb{k}$ is a field of characteristic 0 and $\mathcal{C}$ is a category whose objects are finite-dimensional $\mathbb{k}$-vector spaces. In particular, in [17] it is shown that, for any $g \in \mathcal{G}^{\prime}$, the map

$$
\mathrm{b}(V)=\operatorname{dim}_{\mathbb{k}}(V) /\left(\sum_{V^{\prime} \in \operatorname{irr}\left(\mathcal{C}_{g}\right)} \operatorname{dim}_{\mathbb{k}}\left(V^{\prime}\right)^{2}\right)
$$

is well defined and satisfies all the properties above.

A representative set for $\mathrm{A}$ is a family $\left\{V_{i}\right\}_{i \in I}$ of simple objects of $\mathrm{A}$ numerated by elements of a set $I$ such that any element of $A$ is isomorphic to a unique element of $\left\{V_{i}\right\}_{i \in I}$. Let $I \rightarrow I, i \mapsto i^{*}$ be the involution determined by $V_{i^{*}} \simeq V_{i}^{*}$. For each $g \in \mathcal{G}^{\prime}$ let $I_{g}=\left\{i \in I: V_{i} \in \mathcal{C}_{g}\right\}$, then $I=\bigcup_{g \in \mathcal{G}^{\prime}} I_{g}$. For $i \in I_{g}$, we call $\tilde{i}=g$ the degree of $i$.

By basic data in $\mathcal{C}$ we mean a representative set $\left\{V_{i}\right\}_{i \in I}$ for $\mathrm{A}$ and a family of isomorphisms $\left\{w_{i}: V_{i} \rightarrow V_{i^{*}}^{*}\right\}_{i \in I}$ such that

$$
d_{V_{i}}\left(w_{i^{*}} \otimes \operatorname{Id}_{V_{i}}\right)=d_{V_{i^{*}}}^{\prime}\left(\operatorname{Id}_{V_{i^{*}}} \otimes w_{i}\right): V_{i^{*}} \otimes V_{i} \rightarrow \mathbb{I} \text {. }
$$

Lemma 3 If no object of $\mathrm{A}$ is isomorphic to its dual, then $\mathcal{C}$ contains a basic data. In particular, basic data exists if $\mathcal{X}$ contains the set $\left\{g \in \mathcal{G}: g=g^{-1}\right\}$. 
Proof Choose a representative for each isomorphism class of simple objects of $\bigcup_{g \in \mathcal{G}^{\prime}} \mathcal{C}_{g}$. Let these representatives $\left\{V_{i}\right\}_{i \in I}$ be numerated by a set $I$. Then $I$ is a representative set for $\mathrm{A}$. The hypothesis of the lemma imply that $i^{*} \neq i$ for all $i \in I$. Hence, for any unordered pair $\left(i, i^{*}\right)$, we can take an arbitrary isomorphism $V_{i} \rightarrow\left(V_{i^{*}}\right)^{*}$ for $w_{i}$ and choose $w_{i *}$ so that it satisfies Equation (4).

For the second statement, let $g \in \mathcal{G}^{\prime}$ and let $V$ be a simple object of $\mathcal{C}_{g}$. Since $g^{-1} \neq g$, the $\mathcal{G}$-grading of $\mathcal{C}$ implies that $V$ and $V^{*}$ are not isomorphic. Thus, we can apply the above argument.

If $\mathcal{C}$ is a $(\mathcal{X}, \mathrm{d})$-relative $\mathcal{G}$-spherical category with basic data $\left\{V_{i}, w_{i}\right\}_{i \in I}$ then the functions $\mathrm{d}: \mathrm{A} \rightarrow \mathbb{k}$ and $\mathrm{b}: \mathrm{A} \rightarrow \mathbb{k}$ can and will be considered as functions from $I$ to $\mathbb{k}$ given by $\mathrm{d}(i)=\mathrm{d}\left(V_{i}\right)$ and $\mathrm{b}(i)=\mathrm{b}\left(V_{i}\right)$, respectively.

The following theorem shows that relative $\mathcal{G}$-spherical categories are generalizations of the usual modular categories associated to restricted quantum groups.

Theorem 4 Let $\tilde{U}_{q}(\mathfrak{g})$ be the restricted quantum group associated to a simple Lie algebra $\mathfrak{g}$ of type $A, B, C, D$, at a primitive root of unity $q$ of even order (see [27, XI.6.3]). Let $\mathcal{C}$ be the modular category formed from finite-dimensional representations of $\tilde{U}_{q}(\mathfrak{g})$ modulo negligible morphisms (see $\left.[27, X I]\right)$. Then $\mathcal{C}$ is a $(\varnothing$, qdim)-relative $\mathcal{G}$-spherical category with basic data, where qdim is the usual quantum dimension of $\mathcal{C}$ and $\mathcal{G}=\{1\}$ is the trivial group of one element. Here the map b can be chosen to be the map defined in (3) or the map defined by (3) where the dimension $\operatorname{dim}_{\mathbb{K}}$ is replaced with the usual quantum dimension qdim.

Proof First, $\mathcal{C}$ is a $\mathbb{C}$-linear pivotal category (see [27, XI]). We assign $\mathcal{C}_{1}=\mathcal{C}$ and then by definition $\mathcal{C}$ is $\mathcal{G}$-graded. Also, $\mathcal{C}$ is semisimple with finitely many isomorphism classes of simple objects and so Property (i) of Definition 2 holds. Since qdim is nonzero for each simple object of $\mathcal{C}$ then Equation (1) holds by definition of $G$ and so (A, qdim) is a t-ambi pair. Finally, it is shown in [30] that basic data exists.

Remark 5 In Section 3.4 we show that a relative $\mathcal{G}$-spherical category gives rise to a modified Turaev-Viro invariant. Let $\mathcal{C}$ be the modular category of Theorem 4 . The original Turaev-Viro invariant is not equal to the modified invariant corresponding to $\mathcal{C}$ where $b$ is defined as in Equation (3). However, the original Turaev-Viro invariant is equal to the modified invariant arising from $\mathcal{C}$ when $b$ is taken to be the map given by (3) where the dimension $\operatorname{dim}_{\mathbb{K}}$ is replaced with the usual quantum dimension qdim. In this case, the modified invariant does not depend on the graph in the 3-manifold. 
Remark 6 (About semisimplicity) The main example of relative $\mathcal{G}$-spherical categories we consider in this paper are the categories of modules over the unrestricted quantum groups at roots of unity; see Section 5. These categories are not semisimple which as we explain in this remark is an essential element of these categories. The state sum invariants defined in this paper only use the algebraic data of these category corresponding to their semisimple part $\sum_{g \in \mathcal{G} \backslash \mathcal{X}} \mathcal{D}_{g}$. Roughly speaking, we represent a 3-manifolds with a representation of its fundamental group in $\mathcal{G}$ by a $\mathcal{G}$-colored triangulation. Then the trick consists in using gauge transformations to always avoid the colors in $\mathcal{X}$.

Nevertheless, even if the nonsemisimple modules are never considered, the construction relies on simple modules with vanishing dimension. Such a module $V$ can not exists in a semisimple monoidal category as $q \operatorname{dim}(V):=d_{V} \circ b_{V}^{\prime}=0$ implies that the evaluation map $d_{V}: V^{*} \otimes V \rightarrow \mathbb{I}$ has no section because $\operatorname{Hom}\left(\mathbb{I}, V^{*} \otimes V\right)$ is generated by $b_{V}^{\prime}$. Hence the nonsemisimplicity is essential in all these examples.

\section{$1.4 \hat{\boldsymbol{\Psi}}$-systems}

Here we recall the notion of a $\hat{\Psi}$-system. These systems are the algebraic notions underlying Kashaev's invariant defined in [20]. For more details see [12].

A $\Psi$-system in a $\mathbb{k}$-linear category $\mathcal{C}$ consists of

(i) a distinguished set of simple objects $\left\{V_{i}\right\}_{i \in I}$ such that $\operatorname{Hom}\left(V_{i}, V_{j}\right)=0$ for all $i \neq j$

(ii) an involution $I \rightarrow I, i \mapsto i^{*}$;

(iii) two families of morphisms $\left\{b_{i}: \mathbb{I} \rightarrow V_{i} \otimes V_{i^{*}}\right\}_{i \in I}$ and $\left\{d_{i}: V_{i} \otimes V_{i^{*}} \rightarrow \mathbb{I}\right\}_{i \in I}$, such that for all $i \in I$,

$$
\left(\operatorname{Id}_{V_{i}} \otimes d_{i^{*}}\right)\left(b_{i} \otimes \operatorname{Id}_{V_{i}}\right)=\operatorname{Id}_{V_{i}} \quad \text { and } \quad\left(d_{i} \otimes \operatorname{Id}_{V_{i}}\right)\left(\operatorname{Id}_{V_{i}} \otimes b_{i^{*}}\right)=\operatorname{Id}_{V_{i}} ;
$$

(iv) for any $i, j \in I$ such that $H_{k}^{i j} \neq 0$ for some $k \in I$, the identity morphism $\operatorname{Id}_{V_{i} \otimes V_{j}}$ is in the image of the linear map

$$
\bigoplus_{k \in I} H_{k}^{i j} \otimes_{\mathbb{k}} H_{i j}^{k} \rightarrow \operatorname{End}\left(V_{i} \otimes V_{j}\right), \quad x \otimes y \mapsto x \circ y .
$$

Consider the $\mathbb{k}$-module $H=\widehat{H} \oplus \check{H}$, where

$$
\hat{H}=\bigoplus_{i, j, k \in I} H_{i j}^{k} \quad \text { and } \quad \check{H}=\bigoplus_{i, j, k \in I} H_{k}^{i j} .
$$


Let

$$
\pi_{i j}^{k}: H \rightarrow H_{i j}^{k}, \quad \pi_{k}^{i j}: H \rightarrow H_{k}^{i j}, \quad \hat{\pi}: H \rightarrow \hat{H}, \quad \check{\pi}: H \rightarrow \check{H}
$$

be the obvious projections. The $\mathbb{k}$-module $H$ has a symmetric bilinear pairing $\langle$, given by

$$
\langle x, y\rangle=\sum_{i, j, k \in I}\left(\left\langle\pi_{i j}^{k} x \pi_{k}^{i j} y\right\rangle+\left\langle\pi_{i j}^{k} y \pi_{k}^{i j} x\right\rangle\right) \in \mathbb{k}
$$

for any $x, y \in H$. A transpose of $f \in \operatorname{End}(H)$ is a map $f^{*} \in \operatorname{End}(H)$ such that $\langle f x, y\rangle=\left\langle x, f^{*} y\right\rangle$ for all $x, y \in H$. Note that if a transpose $f^{*}$ of $f$ exists, then it is unique and $\left(f^{*}\right)^{*}=f$ (see [12]). An operator $f \in \operatorname{End}(H)$ such that $f^{*}=f$ is called symmetric. An operator $f \in \operatorname{End}(H)$ such that $f\left(H_{k}^{i j}\right) \subset H_{k}^{i j}$ and $f\left(H_{i j}^{k}\right) \subset H_{i j}^{k}$ for all $i, j, k \in I$ is called grading-preserving.

We define linear maps $A, B: H \rightarrow H$ by

$$
\begin{aligned}
& A x=\sum_{i, j, k \in I}\left(\left(\operatorname{Id}_{V^{*}} \otimes \pi_{i j}^{k} x\right)\left(b_{i^{*}} \otimes \operatorname{Id}_{V_{j}}\right)+\left(d_{i^{*}} \otimes \operatorname{Id}_{V_{j}}\right)\left(\operatorname{Id}_{V_{i}} \otimes \pi_{k}^{i j} x\right)\right), \\
& B x=\sum_{i, j, k \in I}\left(\left(\pi_{i j}^{k} x \otimes \operatorname{Id}_{V^{*}}\right)\left(\operatorname{Id}_{V_{i}} \otimes b_{j}\right)+\left(\operatorname{Id}_{V_{i}} \otimes d_{j}\right)\left(\pi_{k}^{i j} x \otimes \operatorname{Id}_{V^{*}}\right)\right) .
\end{aligned}
$$

In [12], it is shown that both $A$ and $B$ are involutive and have transposes. Also, from [12] we have that the operators

$$
L=A^{*} A, \quad R=B^{*} B, \quad C=(A B)^{3} \quad \text { in } \operatorname{End}(H)
$$

are symmetric, grading-preserving and invertible.

Definition 7 [12] A $\widehat{\Psi}$-system in $\mathcal{C}$ is a $\Psi$-system in $\mathcal{C}$ together with a choice of invertible, symmetric, grading-preserving operators $C^{\frac{1}{2}}, R^{\frac{1}{2}} \in \operatorname{End}(H)$ satisfying

$$
\begin{aligned}
\left(C^{\frac{1}{2}}\right)^{2} & =C, \quad A C^{\frac{1}{2}} A=B C^{\frac{1}{2}} B=C^{-\frac{1}{2}}, \\
\left(R^{\frac{1}{2}}\right)^{2} & =R, \quad B R^{\frac{1}{2}} B=R^{-\frac{1}{2}}, \quad R^{\frac{1}{2}} C^{\frac{1}{2}}=C^{\frac{1}{2}} R^{\frac{1}{2}}, \\
T C_{1}^{\frac{1}{2}} C_{2}^{\frac{1}{2}} & =T C_{3}^{\frac{1}{2}} C_{4}^{\frac{1}{2}}, \quad T R_{1}^{\frac{1}{2}} L_{2}^{\frac{1}{2}}=T R_{3}^{\frac{1}{2}} L_{4}^{\frac{1}{2}}, \\
T R_{1}^{\frac{1}{2}} R_{2}^{\frac{1}{2}} & =T C_{3}^{\frac{1}{2}} R_{4}^{\frac{1}{2}}, \quad T L_{1}^{\frac{1}{2}}=T C_{2}^{\frac{1}{2}} L_{3}^{\frac{1}{2}} L_{4}^{\frac{1}{2}},
\end{aligned}
$$

where $L^{\frac{1}{2}}$ is defined to be the invertible operator $L^{\frac{1}{2}}=B A R^{-\frac{1}{2}} A B \in \operatorname{End}(H)$. 


\section{Modified Reshetikhin-Turaev link invariants from projec- tive ambidextrous objects}

In this section we show that certain projective modules lead to ambidextrous traces and renormalized invariants as discussed in Section 1.2. Let $\mathcal{C}$ be a pivotal $\mathbb{k}$-linear category with duality morphisms $b_{V}: \mathbb{I} \rightarrow V \otimes V^{*}, d_{V}: V^{*} \otimes V \rightarrow \mathbb{I}, b_{V}^{\prime}: \mathbb{I} \rightarrow V^{*} \otimes V$ and $d_{V}^{\prime}: V \otimes V^{*} \rightarrow \mathbb{I}$. For every morphism $f: V \rightarrow W$ in $\mathcal{C}$ let $f^{*}: W^{*} \rightarrow V^{*}$ be the dual (or transposed) morphism given by

$$
f^{*}=\left(d_{W} \otimes \operatorname{Id}_{V^{*}}\right)\left(\operatorname{Id}_{W^{*}} \otimes f \otimes \operatorname{Id}_{V^{*}}\right)\left(\operatorname{Id}_{W^{*}} \otimes b_{V}\right) .
$$

The axioms of a pivotal category imply that for each object $V$ of $\mathcal{C}$ there is a canonical functorial isomorphism $V \rightarrow V^{* *}$; see [18]. To simplify notation we will use this isomorphism to identify $V^{* *}$ with $V$. Therefore, for any object $V$ of $\mathcal{C}$, we have $\left(V \otimes V^{*}\right)^{*}=\left(V^{*}\right)^{*} \otimes V^{*}=V \otimes V^{*}$. A simple object $V$ is ambidextrous if $f \circ b_{V}=$ $f^{*} \circ b_{V}$ for all $f \in \operatorname{End}\left(V \otimes V^{*}\right)$. Note in [18] it is shown that when $\mathcal{C}$ is ribbon this definition is equivalent to the definition of ambidextrous given in [16].

Theorem 8 Let $\mathcal{C}$ be a ribbon category and let $\mathrm{A}_{\text {Proj }}$ be the set of simple projective objects of $\mathcal{C}$. Suppose that there is a projective ambidextrous object in $\mathcal{C}$. Then there is an unique (up to a scalar of $\mathbb{k}^{\times}$) map $\mathrm{d}$ : $A_{\text {Proj }} \rightarrow \mathbb{k}^{\times}$such that ( $A_{\text {Proj }}, d$ ) is an ambidextrous pair. Hence the map $\mathrm{G}^{\prime}: \mathcal{L}_{\mathrm{A}_{\text {Proj }}} \rightarrow \mathbb{k}$ given in Section 1.2 is a well defined isotopy invariant.

Proof From [13, Theorem 3.3.2] we have the existence and uniqueness of a trace on the subcategory of projective objects Proj. As in Section 1.2 this trace defines a function $\mathrm{d}$ : $A_{\text {Proj }} \rightarrow \mathbb{k}^{\times}$. The properties of the trace imply that $\mathrm{d}(V)=\mathrm{d}\left(V^{*}\right)$ for all $V \in A_{\text {Proj }}$ and that $\left(A_{\text {Proj }}, \mathrm{d}\right)$ is an ambi pair. The uniqueness of the trace implies the uniqueness of $d$.

Let $J$ be a projective ambidextrous object in a ribbon category $\mathcal{C}$. Following [16], for any simple objects $V, W$ of $\mathcal{C}$, define

$$
S^{\prime}(V, W)=\left\langle{ }_{V} \frac{\mid \checkmark}{\downarrow}\right|
$$

so for any $V \in \mathrm{A}_{\text {Proj }}$ with $S^{\prime}(J, V)$ invertible, we have that

$$
\mathrm{d}(V)=\mathrm{d}(J) \frac{S^{\prime}(V, J)}{S^{\prime}(J, V)}
$$

because $\mathrm{d}(V) S^{\prime}(J, V)=\mathrm{d}(J) S^{\prime}(V, J)$ is the value of $\mathrm{G}^{\prime}$ on the Hopf link colored by $J$ and $V$. 
There is a large class of examples where the category $\mathcal{C}$ is not semisimple and the usual Reshetikhin-Turaev ribbon functor $G$ restricted to the domain $\mathcal{L}_{\text {Aproj }}$ of $G^{\prime}$ is zero. For example, let $\mathfrak{g}$ be a simple Lie algebra. In Section 5 , for each $r^{\text {th }}$ root of unity $\mathrm{e}^{2 i \pi / r}$, we define an algebra $\mathcal{U}^{H}$ which we call the unrolled Drinfeld-Jimbo quantum group associated to $\mathfrak{g}$. Then in Section 5.3, we introduce a ribbon category $\mathcal{D}^{\theta}$ of nilpotent $\mathcal{U}^{H}$-weight modules which satisfy the hypothesis of Theorem 8 . In particular, we use $S^{\prime}$ to give an explicit formula for the modified quantum dimension $d$ in this case (see Theorem 46). The corresponding invariant $\mathrm{G}^{\prime}$ restricted to framed links colored with projective simple modules can be considered as a generalized colored Alexander invariant which in the case of $\mathfrak{s l}(2)$ is the hierarchy of invariants defined in [1] (see [16, Theorem 35]).

In [7] we will extend the link invariants of Theorem 8 to a modified ReshetikhinTuraev-type 3-manifold invariant. More precisely, in [7] we will give a notion of a relative $\mathcal{G}$-modular category and show that such categories have Kirby colors which give rise to 3-manifold invariants.

\section{Topological invariants from relative $\mathcal{G}$-spherical categories}

In this section we will introduce a state sum (15) of $6 j-$ symbol associated to a relative spherical category. This sum is a topological invariant called the modified Turaev-Viro invariant (cf Theorem 16). Then in Section 3.6 we will show that a relative spherical category induces $\hat{\Psi}$-structures (cf Theorem 20) and that the associated Kashaev-type state sums (18) are equal to the modified Turaev-Viro invariant (cf Theorem 21).

\subsection{Modified $6 j-$-symbols}

In [17] the authors show that certain pivotal categories give rise to modified $6 j$-symbols. In this subsection, we show the techniques of [17] can be applied to the situation of relative spherical categories.

Let $\mathcal{C}$ be a $(\mathcal{X}, \mathrm{d})$-relative $\mathcal{G}$-spherical category with basic data $\left\{V_{i}, w_{i}\right\}_{i \in I}$. For any $i, j, k \in I$, recall the notation $H^{i j k}=\operatorname{Hom}\left(\mathbb{I}, V_{i} \otimes V_{j} \otimes V_{k}\right)$. The $\mathbb{k}$-modules $H^{i j k}, H^{j k i}, H^{k i j}$ are canonically isomorphic. Indeed, let $\sigma(i, j, k)$ be the isomorphism

$$
H^{i j k} \rightarrow H^{j k i}, \quad x \mapsto d_{V_{i}} \circ\left(\operatorname{Id}_{V_{i}^{*}} \otimes x \otimes \operatorname{Id}_{V_{i}}\right) \circ b_{V_{i}}^{\prime} .
$$

Using the functor $\mathrm{G}: \mathrm{Gr}_{\mathcal{C}} \rightarrow \mathcal{C}$, one easily proves that

$$
\sigma(k, i, j) \sigma(j, k, i) \sigma(i, j, k)=\operatorname{Id}_{H^{i j k}} .
$$


Identifying the modules $H^{i j k}, H^{j k i}, H^{k i j}$ along these isomorphisms we obtain a symmetrized multiplicity module $H(i, j, k)$ depending only on the cyclically ordered triple $(i, j, k)$. Remark that the $\mathcal{G}$-grading of $\mathcal{C}$ implies that

$$
\text { for all } i, j, k \in I \text { such that } \tilde{i} \tilde{j} \tilde{k} \neq 1 \in \mathcal{G} \text {, then } H(i, j, k)=\{0\} \text {. }
$$

By a labeling of a graph we mean a function assigning to every edge of the graph an element of $I$. By a trivalent graph we mean a (finite oriented) graph whose vertices all have valency 3 . Let $\Gamma$ be a labeled trivalent graph in $S^{2}$. Using the standard orientation of $S^{2}$ (induced by the right-handed orientation of the unit ball in $\mathbb{R}^{3}$ ), we cyclically order the set $X_{v}$ of 3 half-edges adjacent to any given vertex $v$ of $\Gamma$. The labels of the edges determine a function $f_{v}: X_{v} \rightarrow I$ as follows: if a half-edge $e$ adjacent to $v$ is oriented towards $v$, then $f_{v}(e)=i$ is the label of the edge of $\Gamma$ containing $e$; if a half-edge $e$ adjacent to $v$ is oriented away from $v$, then $f_{v}(e)=i^{*}$. Set $H_{v}(\Gamma)=H\left(f_{v}\right)$ and $H(\Gamma)=\bigotimes_{v} H_{v}(\Gamma)$, where $v$ runs over all vertices of $\Gamma$.

Consider now a labeled trivalent graph $\Gamma \subset S^{2}$ endowed with a family of vectors $h=\left\{h_{v} \in H_{v}(\Gamma)\right\}_{v}$, where $v$ runs over all vertices of $\Gamma$. We thicken $\Gamma$ into a $\mathcal{C}-$ colored ribbon graph on $S^{2}$ as follows. First, we insert inside each edge $e$ of $\Gamma$ a coupon with one edge outgoing from the bottom along $e$ and with one edge outgoing from the top along $e$ in the direction opposite to the one on $e$. If $e$ is labeled with $i \in I$, then these two new (smaller) edges are labeled with $V_{i}$ and $V_{i^{*}}$, respectively, and the coupon is labeled with $w_{i}: V_{i} \rightarrow V_{i^{*}}^{*}$ as in Figure 1 .

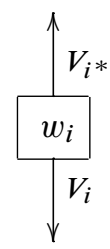

Figure 1

Next, we thicken each vertex $v$ of $\Gamma$ to a coupon so that the three half-edges adjacent to $v$ yield three arrows adjacent to the top side of the coupon and oriented towards it. If $i, j, k \in I$ are the labels of these arrows (enumerated from the left to the right), then we color this coupon with the image of $h_{v}$ under the natural isomorphism $H_{v}(\Gamma) \rightarrow H^{i j k}$. Denote the resulting $\mathcal{C}$-colored ribbon graph by $\Omega_{\Gamma, h}$. Then $\mathbb{G}(\Gamma, h)=\mathrm{G}\left(\Omega_{\Gamma, h}\right)$ is an isotopy invariant of the pair $(\Gamma, h)$ independent of the way in which the vertices of $\Gamma$ are thickened to coupons.

The invariant $G^{\prime}$ defined in Section 1.3 can be extended to a bigger class of $\mathcal{C}$-colored ribbon graphs in $S^{2}$. We say that a coupon of a ribbon graph is straight if both its 
bottom and top sides are incident to exactly one arrow. We can remove a straight coupon and unite the incident arrows into a (longer) edge; see Figure 2. We call this operation straightening. A quasitrivalent ribbon graph is a ribbon graph in $S^{2}$ such that straightening it at all straight coupons we obtain a trivalent ribbon graph.

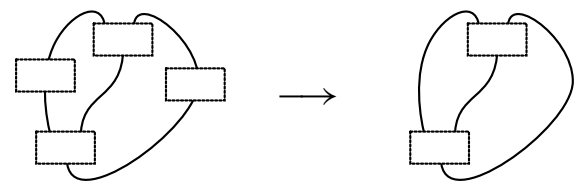

Figure 2: Straightening a quasitrivalent ribbon graph

Lemma 9 Let $\overline{\mathcal{T}_{I}}$ be the class of connected quasitrivalent ribbon graphs in $S^{2}$ such that the colors of all edges belong to the set $\left\{V_{i}\right\}_{i \in I}$ and all straight coupons are colored with isomorphisms in $\mathcal{C}$. Then Equation (2) determines a well-defined function $\mathrm{G}^{\prime}: \overline{\mathcal{T}_{I}} \rightarrow \mathbb{k}$.

Proof This is proved in [17, Lemma 2].

We can combine the invariant $G^{\prime}$ with the thickening of trivalent graphs to obtain invariants of trivalent graphs in $S^{2}$. Suppose that $\Gamma \subset S^{2}$ is a labeled connected trivalent graph. We define

$$
\mathbb{G}^{\prime}(\Gamma) \in H(\Gamma)^{\star}=\operatorname{Hom}_{\mathbb{k}}(H(\Gamma), \mathbb{k})
$$

as follows. Pick any family of vectors $h=\left\{h_{v} \in H_{v}(\Gamma)\right\}_{v}$, where $v$ runs over all vertices of $\Gamma$. The $\mathcal{C}$-colored ribbon graph $\Omega_{\Gamma, h}$ constructed above belongs to the class $\overline{\mathcal{T}_{I}}$ defined in Lemma 9 . Set

$$
\mathbb{G}^{\prime}(\Gamma)\left(\otimes_{v} h_{v}\right)=\mathrm{G}^{\prime}\left(\Omega_{\Gamma, h}\right) \in \mathbb{k} .
$$

By the properties of $\mathrm{G}^{\prime}$, the vector $\mathbb{G}^{\prime}(\Gamma) \in H(\Gamma)^{\star}$ is an isotopy invariant of $\Gamma$. Both $H(\Gamma)$ and $\mathbb{G}^{\prime}(\Gamma)$ are preserved under the reversion transformation inverting the orientation of an edge of $\Gamma$ and replacing the label of this edge, $i$, with $i^{*}$. This can be easily deduced from Equation (4).

Let $i, j, k, l, m, n$ be six elements of $I$. Consider the labeled trivalent graph $\Gamma=$ $\Gamma(i, j, k, l, m, n) \subset \mathbb{R}^{2} \subset S^{2}$ given in Figure 3(a). By definition,

$$
H(\Gamma)=H\left(m, n^{*}, i^{*}\right) \otimes_{\mathbb{k}} H\left(n, l^{*}, j^{*}\right) \otimes_{\mathbb{k}} H\left(i, j, k^{*}\right) \otimes_{\mathbb{k}} H\left(k, l, m^{*}\right) .
$$

We define the modified $6 j$-symbol of the tuple $(i, j, k, l, m, n)$ to be

$$
\left|\begin{array}{ccc}
i & j & k \\
l & m & n
\end{array}\right|=\mathbb{G}^{\prime}(\Gamma) \in H(\Gamma)^{\star} .
$$


It follows from the definitions that the modified $6 j-$ symbols have the symmetries of an oriented tetrahedron. In particular,

$$
\left|\begin{array}{ccc}
i & j & k \\
l & m & n
\end{array}\right|=\left|\begin{array}{ccc}
j & k^{*} & i^{*} \\
m & n & l
\end{array}\right|=\left|\begin{array}{ccc}
k & l & m \\
n^{*} & i & j^{*}
\end{array}\right| \text {. }
$$

These equalities hold because the labeled trivalent graphs in $S^{2}$ defining these $6 j-$ symbols are related by isotopies and reversion transformations described above. Also, it follows from Equation (7) that $H\left(i, j, k^{*}\right)=0$ if $\tilde{i} \tilde{j} \neq \tilde{k}$ and so

$$
\left|\begin{array}{ccc}
i & j & k \\
l & m & n
\end{array}\right|=0
$$

if one of the following equalities is not satisfied in $\mathcal{G}$ :

$$
\tilde{i} \tilde{j}=\tilde{k}, \quad \tilde{k} \tilde{l}=\widetilde{m}, \quad \tilde{j} \tilde{l}=\tilde{n} \quad \text { or } \quad \tilde{i} \tilde{n}=\widetilde{m} .
$$

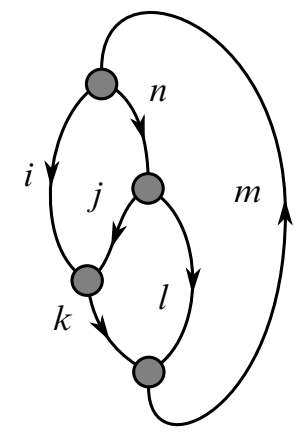

(a) $\Gamma(i, j, k, l, m, n)$

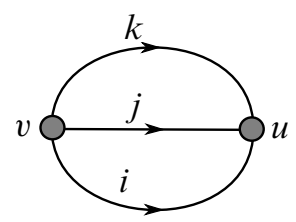

(b) $\Theta(i, j, k)$

Figure 3: Elementary labeled graphs

For any indices $i, j, k \in I$, we define a pairing

$$
\begin{gathered}
(,)_{i j k}: H(i, j, k) \otimes_{\mathbb{k}} H\left(k^{*}, j^{*}, i^{*}\right) \rightarrow \mathbb{k}, \\
(x, y)_{i j k}=\mathbb{G}^{\prime}(\Theta)(x \otimes y),
\end{gathered}
$$

where $x \in H(i, j, k)=H^{i j k}$ and $y \in H\left(k^{*}, j^{*}, i^{*}\right)=H^{k^{*} j^{*} i^{*}}$ and $\Theta=\Theta_{i, j, k}$ is the theta graph with vertices $u, v$ and three edges oriented from $v$ to $u$ and labeled with $i, j, k$; see Figure 3(b). Clearly

$$
H_{u}(\Theta)=H^{i j k}=H(i, j, k) \quad \text { and } \quad H_{v}(\Theta)=H^{k^{*} j^{*} i^{*}}=H\left(k^{*}, j^{*}, i^{*}\right)
$$

so that we can use $x, y$ as the colors of $u, v$, respectively. It follows from the definitions that the pairing $(,)_{i j k}$ is invariant under cyclic permutations of $i, j, k$ and $(x, y)_{i j k}=(y, x)_{k^{*} j^{*} i^{*}}$ for all $x \in H(i, j, k)$ and $y \in H\left(k^{*}, j^{*}, i^{*}\right)$. 
Given indices $i, j, k \in I^{3}$ and a tensor product of several $\mathbb{k}$-modules such that among the factors there is a matched pair $H(i, j, k), H\left(k^{*}, j^{*}, i^{*}\right)$, we may contract any element of this tensor product using the pairing (10). This operation is called the contraction along $H(i, j, k)$ and denoted by $*_{i j k}$. For example, an element $x \otimes y \otimes z \in H(i, j, k) \otimes_{\mathbb{k}} H\left(k^{*}, j^{*}, i^{*}\right) \otimes_{\mathbb{k}} H$, where $H$ is a $\mathbb{k}$-module, contracts into $(x, y) z \in H$.

The rest of this section contains properties of the modified $3 j$ and $6 j-$ symbols defined above.

Lemma 10 For any elements $i, j, k$ of $I$, the pairing (10) is nondegenerate.

Proof Let us assume that $H(i, j, k) \neq 0$ otherwise the lemma is trivial. Then by Equation (7), $V_{i} \otimes V_{j}$ is an object of $\mathcal{C}_{\tilde{k}^{-1}}$ which is semisimple because $\tilde{i} \tilde{j}=\tilde{k}^{-1} \in \mathcal{G}^{\prime}$. Then the lemma follows from [17, Lemma 3]. To show that the hypothesis of the lemma in [17] are satisfied it is enough to show that the pair $(i, j)$ has a quality called good. A pair of indices $(l, m) \in I^{2}$ is called good if $V_{l} \otimes V_{m}$ splits as a direct sum of some $V_{n}$ s (possibly with multiplicities) such that $n \in I$ and $\mathrm{d}\left(V_{n}\right)$ is invertible in $\mathbb{k}$. Hence, by definition of a relative spherical category the pair $(i, j)$ is good and the proof follows [17, Lemma 3].

Theorem 11 (The Biedenharn-Elliott identity) Let $j_{0}, j_{1}, \ldots, j_{8}$ be elements of $I$. Assume that $\widetilde{j}_{2} \widetilde{j}_{3} \notin \mathcal{X}$, then

$$
\begin{aligned}
& \sum_{j \in I_{\tilde{j}_{2}} \tilde{j}_{3}} \mathrm{~d}\left(V_{j}\right) *_{j_{2} j_{3} j * *_{j j_{4} j_{7}^{*}} *_{j_{1}} j_{6}^{*}}\left(\left|\begin{array}{lll}
j_{1} & j_{2} & j_{5} \\
j_{3} & j_{6} & j
\end{array}\right| \otimes\left|\begin{array}{lll}
j_{1} & j & j_{6} \\
j_{4} & j_{0} & j_{7}
\end{array}\right| \otimes\left|\begin{array}{lll}
j_{2} & j_{3} & j \\
j_{4} & j_{7} & j_{8}
\end{array}\right|\right) \\
& =*_{j_{5} j_{8} j_{0}^{*}}\left(\left|\begin{array}{lll}
j_{5} & j_{3} & j_{6} \\
j_{4} & j_{0} & j_{8}
\end{array}\right| \otimes\left|\begin{array}{ccc}
j_{1} & j_{2} & j_{5} \\
j_{8} & j_{0} & j_{7}
\end{array}\right|\right) .
\end{aligned}
$$

Here both sides lie in the tensor product of the six $\mathbb{k}-$ modules

$$
\begin{array}{lll}
H\left(j_{6}, j_{3}^{*}, j_{5}^{*}\right), & H\left(j_{5}, j_{2}^{*}, j_{1}^{*}\right), & H\left(j_{0}, j_{4}^{*}, j_{6}^{*}\right), \\
H\left(j_{1}, j_{7}, j_{0}^{*}\right), & H\left(j_{2}, j_{8}, j_{7}^{*}\right), & H\left(j_{3}, j_{4}, j_{8}^{*}\right) .
\end{array}
$$

Proof The proof follows from [17, Theorem 7]. We now explain why the hypothesis of this theorem are satisfied. First, the definitions above imply that the set $J$ of [17] is equal to $I_{\tilde{j}_{2}} \tilde{j}_{3}$. Second, as in the proof of Lemma 10 certain pairs of elements of $I$ must be good. As above, if $l, m, n \in I$ with $H(l, m, n) \neq 0$ then the pair $(l, m)$ is good. Moreover, if the pair $(l, m)$ is not good then for any $n \in I$ the space $H(l, m, n)$ is zero. Thus, from (9) it follows that both sides of Equation (12) are zero unless all required pairs are good and so the hypothesis of [17] are satisfied. 
Theorem 12 (The orthonormality relation) Let $i, j, k, l, m, p$ be elements of $I$. Assume $\tilde{j} \tilde{l} \notin \mathcal{X}$, then

$\mathrm{d}\left(V_{k}\right) \sum_{n \in I_{\tilde{j} \tilde{l}}} \mathrm{~d}\left(V_{n}\right) *_{i n m^{*}} *_{j l n} n^{*}\left(\left|\begin{array}{ccc}i & j & p \\ l & m & n\end{array}\right| \otimes\left|\begin{array}{ccc}k & j^{*} & i \\ n & m & l\end{array}\right|\right)=\delta_{k, p} \operatorname{Id}\left(i, j, k^{*}\right) \operatorname{Id}\left(k, l, m^{*}\right)$,

where $\delta_{k, p}$ is the Kronecker symbol and $\operatorname{Id}(a, b, c)$ is the canonical element of $H(a, b, c) \otimes_{\mathbb{k}} H\left(c^{*}, b^{*}, a^{*}\right)$ determined by the duality pairing.

Proof The proof follows from [17, Theorem 8]. Again here, as in the proof of Theorem 11 the definition of a relative $\mathcal{G}$-spherical category implies the hypothesis of [17].

The following lemma is an algebraic analog of the $H$-bubble move.

Lemma 13 Let $g_{1}, g_{2}, g_{3}, g_{4}, g_{5}, g_{6} \in \mathcal{G} \backslash \mathcal{X}$ with $g_{3}=g_{1} g_{2}, g_{6}=g_{2} g_{4}$ and $g_{5}=g_{1} g_{6}$. If $i \in I_{g_{1}}, j \in I_{g_{2}}, k \in I_{g_{3}}$, then

$$
\begin{array}{r}
\mathrm{d}(k) \sum_{\substack{l \in I_{g_{4}} \\
m \in I_{g_{5}}, n \in I_{g_{6}}}} \mathrm{~d}(n) \mathrm{b}(l) \mathrm{b}(m) * k l m^{*} * i n m^{*} * j l n *\left(\left|\begin{array}{ccc}
i & j & k \\
l & m & n
\end{array}\right| \otimes\left|\begin{array}{lll}
k & j^{*} & i \\
n & m & l
\end{array}\right|\right) \\
=\mathrm{b}(k) \operatorname{Id}\left(i, j, k^{*}\right) .
\end{array}
$$

Proof The proof follows from a direct manipulation of the orthonormality relation using the properties of the map b. In particular, the proof of [17, Lemma 25] can easily be adapted to the case when $\mathcal{G}$ is not commutative.

\subsection{Topological preliminary}

Let $M$ be an oriented compact smooth 3-manifold. Here a graph $Y$ is a finite 1dimensional $\mathrm{CW}$-complex disjoint union a finite number of circles without vertices. We denote by $Y_{0}$ the set of vertices of $Y$ and $\partial Y$ the set of univalent vertices of $Y$. By a graph in $M$, we mean an embedding of a graph in $M$ such that $Y \cap \partial M \subset \partial Y$ and $Y$ meets all connected components of $M$. We say that $Y$ is rooted in $Y \cap \partial M$.

By a triangulation $\mathcal{T}$ of $M$, we mean a smooth $\Delta$-complex structure on $M$ (as in [19]). Loosely speaking, a $\Delta$-complex structure is a quotient space of a collection of disjoint simplices obtained by identifying certain of their faces. In particular, the interior of the simplices of $\mathcal{T}$ are embedded in $M$ but their faces are not necessarily distinct and two different simplices might meet on several faces. We say that $\mathcal{T}$ is quasiregular if any simplex of $\mathcal{T}$ is embedded in $M$. This is equivalent to requiring that the two endpoints of any edge of $\mathcal{T}$ are distinct vertices of $\mathcal{T}$. 
Let $Y$ be a graph in $M$. Let $\mathcal{T}$ be a quasiregular triangulation of $M$ and $\mathcal{T}^{\partial}$ be a triangulation of $\partial M$. Let $\mathcal{Y}$ be a set of unoriented edges of $\mathcal{T}$ such that the union of these edges is the graph $Y$ in $M$. We say the pair $(\mathcal{T}, \mathcal{Y})$ is an $H$-triangulation of $(M, Y)$ relative to $\mathcal{T}^{\partial}$ if the following four conditions hold: (1) the vertices of $Y$ are also vertices of $\mathcal{T}$, (2) all the vertices of $\mathcal{T}$ are contained in $Y$ (ie all the vertices are incident to an edge of $\mathcal{Y}$ ), (3) any edge of $\mathcal{T}$ with both endpoints in $Y_{0}$ is contained in $\partial M$ and (4) $\mathcal{T}$ restricts to $\mathcal{T}^{\partial}$.

Theorem 14 Any triplet (a compact orientable 3-manifold $M$, a quasiregular triangulation $\mathcal{T}^{\partial}$ of $\partial M$, a graph $Y$ in $M$ rooted in $\mathcal{T}^{\partial}$ ) admits an $H$-triangulation of $(M, Y)$ relative to $\mathcal{T}^{\partial}$.

The proof of Theorem 14 will be given in Section 4.3.

\subsection{Fundamental groupoid and space of representations in $\mathcal{G}$}

A groupoid is a small category in which all morphisms are isomorphisms. Let us consider two examples of groupoids. First, every group $\mathcal{G}$ can be seen as a groupoid with only one object whose set of endomorphisms is the group $\mathcal{G}$. Second, let $W$ be a nonempty locally path connected topological space. The fundamental groupoid $\pi_{1}(W)$ of $W$ is the small category whose objects are points of $W$ and whose morphisms are homotopy classes of paths in $W$, where composition is concatenation of paths. Let $Z$ be a subset of $W$. If $Z$ is nonempty, let $\pi_{1}(W, Z)$ be the full subcategory of $\pi_{1}(W)$ whose objects are points of $Z$. When $Z$ is a single point $w$ of $W$ then $\pi_{1}(W, Z)=\pi_{1}(W,\{w\})$ is the usual fundamental group. If $W_{1}, \ldots, W_{n}$ are the connected components of $W$ and $Z$ meets all of these component, then $\pi_{1}(W, Z)=$ $\bigsqcup_{i} \pi_{1}\left(W_{i}, Z \cap W_{i}\right)$.

When $W$ is connected, let

$$
\mathcal{M}(W, \mathcal{G})=\operatorname{Hom}_{\text {Groups }}\left(\pi_{1}(W,\{w\}), \mathcal{G}\right) / \mathcal{G},
$$

where $w$ is any point of $W$ and $g \in \mathcal{G}$ acts on $\rho: \pi_{1}(W,\{w\}) \rightarrow \mathcal{G}$ by conjugation: $g . \rho: \gamma \mapsto g \rho(\gamma) g^{-1}$. The set $\mathcal{M}(W, \mathcal{G})$ is called the space of representations of $W$. Remark that for two different choices of the base point $w$, the corresponding spaces of representations of $W$ are canonically isomorphic. We set

$$
\mathcal{M}(W, Z, \mathcal{G})= \begin{cases}\mathcal{M}(W, \mathcal{G}) & \text { if } Z=\varnothing, \\ \operatorname{Hom}_{\text {groupoid }}\left(\pi_{1}(W, Z), \mathcal{G}\right) & \text { if } Z \neq \varnothing\end{cases}
$$

We extend this definition for nonconnected spaces by

$$
\mathcal{M}\left(\bigsqcup_{i} W_{i}, Z\right)=\prod_{i} \mathcal{M}\left(W_{i}, Z \cap W_{i}, \mathcal{G}\right)
$$


The assignment $(W, Z) \mapsto \mathcal{M}(W, Z, \mathcal{G})$ extends to a contravariant functor. In particular, if $W^{\prime} \subset W$ and $Z^{\prime} \subset Z$ by functoriality we have a map $\mathcal{M}(W, Z, \mathcal{G}) \rightarrow$ $\mathcal{M}\left(W^{\prime}, Z^{\prime}, \mathcal{G}\right)$ called the restriction map. Let $E$ be a set of paths in $\pi_{1}(W, Z)$, we say that $\rho \in \mathcal{M}(W, Z, \mathcal{G})$ is $E$-admissible if $\rho(E) \cap \mathcal{X}=\varnothing$.

Let $W$ be a triangulated manifold. A $\mathcal{G}$-coloring of $W$ is a map $\Phi$ from the set of oriented edges of $W$ to $\mathcal{G}$ such that:

(i) $\Phi(-e)=\Phi(e)^{-1}$ for any oriented edge $e$ of $W$, where $-e$ is $e$ with opposite orientation,

(ii) if $e_{1}, e_{2}, e_{3}$ are ordered edges of a face of $W$ endowed with orientation induced by the order, then $\Phi\left(e_{1}\right) \Phi\left(e_{2}\right) \Phi\left(e_{3}\right)=1$.

If $W_{0}$ is the set of vertices of $W$ then $\Phi \in \mathcal{M}\left(W, W_{0}, \mathcal{G}\right)$ is equivalent to a $\mathcal{G}$-coloring $\Phi$ of $W$. A $\mathcal{G}$-coloring of $W, \Phi \in \mathcal{M}\left(W, W_{0}, \mathcal{G}\right)$ is admissible if it is $W_{1}$-admissible where $W_{1}$ is the set of oriented edges of $W$.

Let $M$ be an oriented compact smooth 3-manifold and $\mathcal{T}^{\partial}$ a quasiregular triangulation of its boundary. Let $Y$ be a graph in $M$ rooted in $\mathcal{T}^{\partial}$. Let $Y_{0}$ be the set of vertices of $Y$ and $\mathcal{T}_{1}^{\partial}$ be the set of edges of $\mathcal{T}^{\partial}$. An admissible representation of $\left(M, \mathcal{T}^{\partial}, Y\right)$ in $\mathcal{G}$ is a $\mathcal{T}_{1}^{\partial}$-admissible element of $\mathcal{M}\left(M, Y_{0}, \mathcal{G}\right)$. If $(\mathcal{T}, \mathcal{Y})$ is a $H$-triangulation of $(M, Y)$ relative to $\mathcal{T}^{\partial}$ then a $\mathcal{G}$-coloring $\Phi \in \mathcal{M}\left(M, \mathcal{T}_{0}, \mathcal{G}\right)$ of $\mathcal{T}$ restricts to an element of $\mathcal{M}\left(M, Y_{0}, \mathcal{G}\right)$.

In particular, this is true for a closed 3-manifold: If $L$ is a link in a closed 3-manifold $M$, and if $(\mathcal{T}, \mathcal{L})$ is a $H$-triangulation of $(M, L)$, then any $\mathcal{G}$-coloring of $\mathcal{T}$ restricts to a (always admissible) representation of the fundamental group of $M$ up to conjugation, ie an element of $\mathcal{M}(M, \mathcal{G})$. This restriction map is the image by the contravariant functor $\mathcal{M}(*, \mathcal{G})$ of the inclusion $(M, \varnothing) \subset\left(M, \mathcal{T}_{0}\right)$.

Theorem 15 Let $(\mathcal{T}, \mathcal{Y})$ be a $H$-triangulation of $(M, Y)$ relative to $\mathcal{T}^{\partial}$. Then for any admissible representation $\rho$ of $\left(M, \mathcal{T}^{\partial}, Y\right)$, there exists an admissible $\mathcal{G}$-coloring of $\mathcal{T}$ which restricts to $\rho$.

The proof of the Theorem 15 is given in Section 4.3.

\subsection{Modified Turaev-Viro invariants}

We start from the algebraic data described in the Section 3.1 and produce a topological invariant of a quadruple $\left(M, \mathcal{T}^{\partial}, Y, \rho\right)$, where $M$ is a compact oriented 3-manifold, $\mathcal{T}^{\partial}$ is a quasiregular triangulation of its boundary, $Y \subset M$ is a graph in $M$ rooted in $\mathcal{T}^{\partial}$, and $\rho$ is an admissible representation of $\left(M, \mathcal{T}^{\partial}, Y\right)$. 
A state of an admissible $\mathcal{G}$-coloring $\Phi$ of a triangulated manifold $W$ is a map $\varphi$ assigning to every oriented edge $e$ of $W$ an element $\varphi(e)$ of $I_{\Phi(e)}$ such that $\varphi(-e)=$ $\varphi(e)^{*}$ for all $e$. The set of all states of $\Phi$ is denoted $\operatorname{St}(\Phi)$. The identities $\mathrm{d}(\varphi(e))=$ $\mathrm{d}(\varphi(-e))$ and $\mathrm{b}(\varphi(e))=\mathrm{b}(\varphi(-e))$ allow us to use the notation $\mathrm{d}(\varphi(e))$ and $\mathrm{b}(\varphi(e))$ for nonoriented edges.

The representation $\rho$ restricts to an admissible $\mathcal{G}$-coloring $\Phi^{\partial}$ of the triangulated surface $\left(\partial M, \mathcal{T}^{\partial}\right)$. Given a state $\varphi$ of the $\mathcal{G}$-coloring $\Phi^{\partial}$ of $\mathcal{T}^{\partial}$, the trivalent graph $\Gamma_{\varphi}$ dual to the 1 -skeleton of $\mathcal{T}^{\partial}$ becomes a labeled graph in an oriented surface. We adopt the convention that an oriented edge $e$ of $\Gamma_{\varphi}$ has the same label than the oriented edge of $\mathcal{T}^{\partial}$ crossing $e$ from the left to the right. We set

$$
H\left(\partial M, \mathcal{T}^{\partial}, \Phi^{\partial}\right)=\bigoplus_{\varphi \in \operatorname{St}\left(\Phi^{\partial}\right)} H\left(\Gamma_{\varphi}\right),
$$

where $H\left(\Gamma_{\varphi}\right)$ is defined in Section 3.1. If $\partial M=\varnothing$ then

$$
\mathcal{T}^{\partial}=\Gamma=\varnothing \quad \text { and } \quad H\left(\partial M, \mathcal{T}^{\partial}, \Phi^{\partial}\right)=\mathbb{k} .
$$

Let $(\mathcal{T}, \mathcal{Y})$ be an $H$-triangulation of $(M, Y)$ relative to $\mathcal{T}^{\partial}$ and let $\Phi$ be an admissible $\mathcal{G}$-coloring of $\mathcal{T}$ that restrict to $\rho$. We now define a certain partition function (state sum) as follows. For each tetrahedron $t$ of $\mathcal{T}$, we choose its vertices $v_{1}, v_{2}, v_{3}, v_{4}$ so that the (ordered) triple of oriented edges $\left(\overrightarrow{v_{1} v_{2}}, \overrightarrow{v_{1} v_{3}}, \overrightarrow{v_{1} v_{4}}\right)$ is positively oriented with respect to the orientation of $M$. Here by $\overrightarrow{v_{1} v_{2}}$ we mean the edge oriented from $v_{1}$ to $v_{2}$, etc. For each $\varphi \in \operatorname{St}(\Phi)$, set

$$
|t|_{\varphi}=\left|\begin{array}{lll}
i & j & k \\
l & m & n
\end{array}\right| \quad \text { where }\left\{\begin{array}{lll}
i=\varphi\left(\overrightarrow{v_{2} v_{1}}\right), & j=\varphi\left(\overrightarrow{v_{3} v_{2}}\right), & k=\varphi\left(\overrightarrow{v_{3} v_{1}}\right) \\
l=\varphi\left(\overrightarrow{v_{4} v_{3}}\right), & m=\varphi\left(\overrightarrow{v_{4} v_{1}}\right), & n=\varphi\left(\overrightarrow{v_{4} v_{2}}\right)
\end{array}\right.
$$

This $6 j$-symbol belongs to the tensor product of 4 multiplicity modules associated to the faces of $t$ and does not depend on the choice of the numeration of the vertices of $t$ compatible with the orientation of $M$. Note that any face of $\mathcal{T} \backslash \mathcal{T}^{\partial}$ belongs to exactly two tetrahedra of $\mathcal{T}$, and the associated multiplicity modules are dual to each other. The tensor product of the $6 j$-symbols $|t|_{\varphi}$ associated to all tetrahedra $t$ of $\mathcal{T}$ can be contracted using this duality. We denote by cntr the tensor product of all these contractions. Let $\mathcal{T}_{1}^{\prime}$ be the set of unoriented edges of $\mathcal{T}$ that are not in $\mathcal{T}^{\partial}$ and let $\mathcal{T}_{3}$ the set of tetrahedra of $\mathcal{T}$. Set

$$
\begin{aligned}
& \operatorname{TV}(\mathcal{T}, \mathcal{Y}, \Phi) \\
& =\sum_{\varphi \in \operatorname{St}(\Phi)} \prod_{e \in \mathcal{T}_{1}^{\prime} \backslash \mathcal{Y}} \mathrm{d}(\varphi(e)) \prod_{e \in \mathcal{Y}} \mathrm{b}(\varphi(e)) \operatorname{cntr}\left(\bigotimes_{t \in \mathcal{T}_{3}}|t|_{\varphi}\right) \in H\left(\mathcal{T}^{\partial}, \Phi^{\partial}\right) .
\end{aligned}
$$


Theorem $16 \operatorname{TV}(\mathcal{T}, \mathcal{Y}, \Phi)$ depends only on the isotopy class of $Y$ in $M$, on the triangulation $\mathcal{T}^{\partial}$ of $\partial M$ and on the admissible representation $\rho$ of $\left(M, \mathcal{T}^{\partial}, Y\right)$. We set

$$
T V(M, Y, \rho)=T V(\mathcal{T}, \mathcal{Y}, \Phi) \in H\left(\partial M, \mathcal{T}^{\partial}, \Phi^{\partial}\right) .
$$

Moreover, this invariant extends to a relative quantum field theory given in (31).

A proof of this theorem will be given in Section 4.3.

\subsection{Kashaev-type 3-manifold invariants}

In [12], Geer, Kashaev and Turaev introduce the notion of a $\hat{\Psi}$-system in a tensor category (see Definition 7 above). It is shown that such a system, plus some algebraic data, leads to the existence of a Kashaev-type invariant of a link in a 3-manifold. In this subsection we briefly recall the construction of this invariant given in [12].

Throughout this subsection, $M$ is a closed connected orientable 3 -manifold and $L$ is a nonempty link inside $M$. Here we regard $L$ as a graph in $M$ with no vertices. Let $(\mathcal{T}, \mathcal{L})$ be an $H$-triangulation of $(M, L)$. Let $t$ be an tetrahedron of $\mathcal{T}$. An integral charge on $t$ is a $\frac{1}{2} \mathbb{Z}$-valued map $c$ defined on the edges of $t$ such that $c(e)=c\left(e^{\prime}\right)$ for opposite edges $e$ and $e^{\prime}$, and for any edges $e_{1}, e_{2}, e_{3}$ of $t$ forming the boundary of a face of $t$ we have $c\left(e_{1}\right)+c\left(e_{2}\right)+c\left(e_{3}\right)=\frac{1}{2}$.

Let $E(\mathcal{T})$ be the set of edges of $\mathcal{T}$ and let $\widehat{E}(\mathcal{T})$ be the set consisting of the edges of all the tetrahedra $\left\{t_{i}\right\}$ of $\mathcal{T}$. Any edge $e$ of $\mathcal{T}$ gives rise to $n$ elements of $\widehat{E}(\mathcal{T})$ where $n$ is the number of tetrahedra of $\mathcal{T}$ adjacent to $e$. Let $\epsilon_{\mathcal{T}}: \widehat{E}(\mathcal{T}) \rightarrow E(\mathcal{T})$ be the natural projection. An integral charge on an $H$-triangulation $(\mathcal{T}, \mathcal{L})$ of $(M, L)$ is a map $c: \widehat{E}(\mathcal{T}) \rightarrow \frac{1}{2} \mathbb{Z}$ such that the restriction of $c$ to any tetrahedron $t$ of $\mathcal{T}$ is an integral charge on $t$, and for each edge $e$ of $\mathcal{T}$ we have $\sum_{e^{\prime} \in \epsilon_{\mathcal{T}}^{-1}(e)} c\left(e^{\prime}\right)=c_{e}$, where $c_{e}=0$, if $e$ belongs to $\mathcal{L}$ and $c_{e}=1$, otherwise. Each charge $c$ on $(\mathcal{T}, \mathcal{L})$ determines a cohomology class $[c] \in H^{1}(M ; \mathbb{Z} / 2 \mathbb{Z})$ as follows. Let $s$ be a simple closed curve in $M$ which lies in general position with respect to $\mathcal{T}$ and such that $s$ never leaves a tetrahedron $t$ of $\mathcal{T}$ through the same 2 -face by which it entered. Thus, each time $s$ passes through $t$, it determines a unique edge $e$ belonging to both the entering and departing faces. The sum of the residues $\left.2 c\right|_{t}(e)(\bmod 2) \in \mathbb{Z} / 2 \mathbb{Z}$ over all passages of $s$ through tetrahedra of $\mathcal{T}$ depends only on the homology class of $s$ and is the value of $[c]$ on $s$. For any nonempty link $L \subset M$ and any $\zeta \in H^{1}(M ; \mathbb{Z} / 2 \mathbb{Z})$, each $H$-triangulation of $(M, L)$ has a charge representing $\zeta$. The theory of integral charges is based on the work of Neumann [24; 25].

We now describe the algebraic data needed to define the Kashaev-type invariant. Let $\mathcal{C}$ be a $\mathbb{k}$-linear category where $\mathbb{k}$ is a field. Recall the vector spaces $H_{k}^{i j}, H_{j k}^{i}, H$, etc, 
from Section 1. Fix a $\widehat{\Psi}$-system in $\mathcal{C}$ with distinguished simple objects $\left\{V_{i}\right\}_{i \in I}$. Fix a family $\left\{I_{g}\right\}_{g \in \mathcal{G}}$ of finite subsets of the set $I$ numerated by elements of a group $\mathcal{G}$ and satisfying the following conditions:

(i) For any $g \in \mathcal{G}$, if $i \in I_{g}$, then $i^{*} \in I_{g^{-1}}$.

(ii) For any $i_{1} \in I_{g_{1}}, i_{2} \in I_{g_{2}}, k \in I \backslash I_{g_{1}} g_{2}$ with $g_{1}, g_{2} \in \mathcal{G}$, we have $H_{k}^{i_{1} i_{2}}=0$.

(iii) If $i_{1} \in I_{g_{1}}, i_{2} \in I_{g_{2}}$ with $g_{1}, g_{2} \in \mathcal{G}$ then either $I_{g_{1} g_{2}}=\varnothing$ or there is a $k \in I_{g_{1}} g_{2}$ such that $H_{k}^{i_{1} i_{2}} \neq 0$.

(iv) For any finite family $\left\{g_{r} \in \mathcal{G}\right\}_{r}$, there is $g \in \mathcal{G}$ such that $I_{g g_{r}} \neq \varnothing$ for all $r$.

(v) We are given a map $\mathrm{b}: I \rightarrow \mathbb{k}$ such that $\mathrm{b}(i)=\mathrm{b}\left(i^{*}\right)$ for all $i \in I$, and for any $g_{1}, g_{2} \in \mathcal{G}, k \in I_{g_{1} g_{2}}$ such that $I_{g_{1}} \neq \varnothing$ and $I_{g_{2}} \neq \varnothing$,

$$
\sum_{i_{1} \in I_{g_{1}}, i_{2} \in I_{g_{2}}} \mathrm{~b}\left(i_{1}\right) \mathrm{b}\left(i_{2}\right) \operatorname{dim}_{\mathbb{k}}\left(H_{k}^{i_{1} i_{2}}\right)=\mathrm{b}(k) .
$$

We define two linear forms $T, \bar{T},: H^{\otimes 4} \rightarrow \mathbb{k}$ by the following diagrammatic formulae: for any $u, v, x, y \in H$,

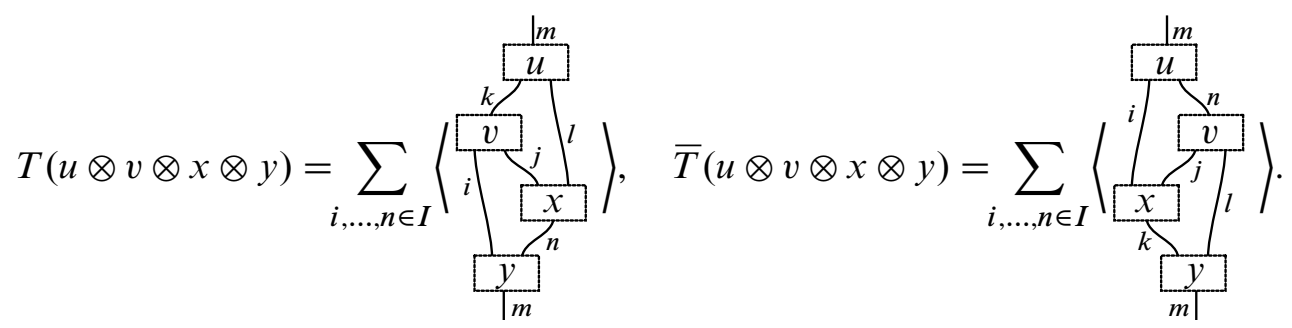

For any $a, b \in \frac{1}{2} \mathbb{Z}$, let $T(a, b), \bar{T}(a, b): H^{\otimes 4} \rightarrow \mathbb{k}$ be linear maps

$$
T(a, b)=T q_{1}^{4 a b} R_{1}^{b} R_{2}^{-a} L_{3}^{-a} R_{3}^{-b}, \quad \bar{T}(a, b)=\bar{T} q_{1}^{-4 a b} L_{2}^{-a} R_{2}^{-b} R_{3}^{-a} R_{4}^{b} .
$$

Let $q \in \operatorname{End}(H)$ be the operator $q=R^{\frac{1}{2}} A R^{-\frac{1}{2}} A L^{-\frac{1}{2}} C^{-\frac{1}{2}}$.

Let $(\mathcal{T}, \mathcal{L})$ be an $H$-triangulation of $(M, L)$, with integral charge $c$. Let $\Phi$ be a $\mathcal{G}$-coloring of $\mathcal{T}$ such that $\operatorname{St}(\Phi) \neq \varnothing$. As remarked in the previous subsection, the $\mathcal{G}$-coloring $\Phi$ induces the conjugacy class of a representation $[\Phi] \in \mathcal{M}(M, \mathcal{G})$. Fix a total ordering of the vertices of $\mathcal{T}$. We recall the partition function defined, in [12], from the above data.

For any tetrahedron $t$ of $\mathcal{T}$, let $v_{1}, v_{2}, v_{3}, v_{4}$ be its vertices in increasing order (induced from the total ordering). We say that $t$ is right oriented if the tangent vectors 
$v_{1} v_{2}, v_{1} v_{3}, v_{1} v_{4}$ form a positive basis in the tangent space of $M$; otherwise $t$ is left oriented. For each state $\varphi$ of $\Phi$, set

$$
\begin{aligned}
& i=\varphi\left(\overrightarrow{v_{1} v_{2}}\right), \quad j=\varphi\left(\overrightarrow{v_{2} v_{3}}\right), \quad k=\varphi\left(\overrightarrow{v_{1} v_{3}}\right), \\
& l=\varphi\left(\overrightarrow{v_{3} v_{4}}\right), \quad m=\varphi\left(\overrightarrow{v_{1} v_{4}}\right), \quad n=\varphi\left(\overrightarrow{v_{2} v_{4}}\right) \text {, }
\end{aligned}
$$

where $\overrightarrow{v_{i} v_{j}}$ is the oriented edge of $t$ going from $v_{i}$ to $v_{j}$. Then for each right oriented tetrahedron $t$ of $\mathcal{T}$ and state $\varphi$ the restriction of $T\left(c\left(v_{1} v_{2}\right), c\left(v_{2} v_{3}\right)\right)$ to the tensor product $H_{k l}^{m} \otimes_{\mathbb{k}} H_{i j}^{k} \otimes_{\mathbb{k}} H_{n}^{j l} \otimes_{\mathbb{k}} H_{m}^{i n} \subset H^{\otimes 4}$ gives a vector in the $\mathbb{k}$-vector space

$$
\operatorname{Hom}_{\mathbb{k}}\left(H_{k l}^{m} \otimes_{\mathbb{k}} H_{i j}^{k} \otimes_{\mathbb{k}} H_{n}^{j l} \otimes_{\mathbb{k}} H_{m}^{i n}, \mathbb{k}\right)=H_{m}^{k l} \otimes_{\mathbb{k}} H_{k}^{i j} \otimes_{\mathbb{k}} H_{j l}^{n} \otimes_{\mathbb{k}} H_{i n}^{m} .
$$

This vector is denoted by $|t|_{\varphi}^{c}$ and depends on $\varphi, c$ and the ordering of the vertices of $\mathcal{T}$. Similarly, if $t$ a left oriented tetrahedron then one uses $\bar{T}\left(c\left(v_{1} v_{2}\right), c\left(v_{2} v_{3}\right)\right)$ to assigns $t$ a vector $|t|_{\varphi}^{c} \in H_{m}^{i n} \otimes_{\mathbb{k}} H_{n}^{j l} \otimes_{\mathbb{k}} H_{i j}^{k} \otimes_{\mathbb{k}} H_{k l}^{m}$. The multiplicity modules of $|t|_{\varphi}^{c}$ are associated to the faces of $t$.

Note that any face of $\mathcal{T}$ belongs to exactly two tetrahedra of $\mathcal{T}$, and the associated multiplicity modules are dual to each other as in [12, Lemma 1]. The tensor product of the $6 j$-symbols $|t|_{\varphi}^{c}$ associated to all tetrahedra $t$ of $\mathcal{T}$ can be contracted using this duality. We denote by cntr the tensor product of all these contractions. Set

$$
\mathrm{K}(\mathcal{T}, \mathcal{L}, \Phi, c)=\sum_{\varphi \in \operatorname{St}(\Phi)}\left(\prod_{e \in \mathcal{L}} \mathrm{b}(\varphi(e))\right) \operatorname{cntr}\left(\bigotimes_{t}|t|_{\varphi}^{c}\right) \in \mathbb{k}
$$

where $t$ runs over all tetrahedra of $\mathcal{T}$.

Theorem 17 [12] Suppose that there exists a scalar $\widetilde{q} \in \mathbb{k}$ such that $q$ is equal to the operator

$$
\widetilde{q} \operatorname{Id}_{\widehat{H}} \oplus \tilde{q}^{-1} \operatorname{Id}_{\check{H}} \in \operatorname{End}(H) .
$$

Then, up to multiplication by integer powers of $\widetilde{q}$, the state $\operatorname{sum} \mathrm{K}(\mathcal{T}, \mathcal{L}, \Phi, c)$ depends only on $[\Phi] \in \mathcal{M}(M, \mathcal{G})$, the isotopy class of $L$ in $M$, and the cohomology class $[c]=\zeta \in H^{1}(M ; \mathbb{Z} / 2 \mathbb{Z})$. We set $\mathrm{K}(M, L,[\Phi], \zeta)=\mathrm{K}(\mathcal{T}, \mathcal{L}, \Phi, c)$.

\section{6 $\widehat{\Psi}$-systems in relative $\mathcal{G}$-spherical categories}

In this subsection we prove that a relative $\mathcal{G}$-spherical category with basic data has a natural $\hat{\Psi}$-system for any choice of a square root of the modified dimension $d$. Moreover, for this situation, we show that corresponding Kashaev-type and modified TV invariants are equal. 
Let $\mathcal{C}$ be a $(\mathcal{X}, \mathrm{d})$-relative $\mathcal{G}$-spherical category with basic data $\left\{V_{i}, w_{i}\right\}_{i \in I}$ where the ground ring $\mathbb{k}$ is a field. For every pair $\left(i, i^{*}\right) \in I \times I$ define the morphisms $d_{i}, d_{i *}, b_{i}, b_{i *}$ by

$$
\begin{aligned}
d_{i}=d_{V_{i^{*}}}\left(w_{i} \otimes \operatorname{Id}_{V_{i^{*}}}\right), & d_{i^{*}}=d_{V_{i^{*}}}^{\prime}\left(\operatorname{Id}_{V_{i^{*}}} \otimes w_{i}\right), \\
b_{i}=\left(\operatorname{Id}_{V_{i}} \otimes w_{i^{*}}^{-1}\right) \circ b_{V_{i}}, & b_{i^{*}}=\left(w_{i^{*}}^{-1} \otimes \operatorname{Id}_{V_{i}}\right) b_{V_{i}}^{\prime} .
\end{aligned}
$$

It is easy to see that Equation (4) implies $\left(\operatorname{Id}_{V_{i}} \otimes w_{i^{*}}^{-1}\right) b_{V_{i}}=\left(w_{i}^{-1} \otimes \operatorname{Id}_{V_{i^{*}}}\right) b_{V_{i^{*}}}^{\prime}$. Therefore, if $i=i^{*}$ then $b_{i}=b_{i^{*}}$ and $d_{i}=d_{i^{*}}$.

Recall the definition of a $\Psi$-system given in Section 1.4.

Proposition 18 The collection $\left\{V_{i}, b_{i}, d_{i}\right\}_{i \in I}$ is a $\Psi$-system in $\mathcal{C}$.

Proof By definition $\left\{V_{i}, b_{i}, d_{i}\right\}_{i \in I}$ satisfies the first three properties in the definition of a $\Psi$-system. Let $i, j \in I$ and suppose $H_{k}^{i j} \neq 0$ for some $k$. Then Equation (7) implies $\widetilde{i} \widetilde{j}=\tilde{k} \in \mathcal{G}^{\prime}$ and so $V_{i} \otimes V_{j}$ is an object of $\mathcal{C}_{k}$. Thus, since $\mathcal{C} \widetilde{k}$ is semisimple with a finite number of isomorphism classes of simple objects given by $\left\{V_{l}: l \in I_{\tilde{k}}\right\}$ we have that $\operatorname{Id}_{V_{i} \otimes V_{j}}$ has the desired property.

Recall the operators $A, B, L=A^{*} A, R=B^{*} B: H \rightarrow H$ defined in Section 1.4. Since $\mathcal{C}$ is pivotal we have $A B A=B A B$ and thus $C=(A B)^{3}=\operatorname{Id}_{H}$. Let $S=A B A$ then $S^{*}=S=S^{-1}$ and $S A S=B$.

Choose a function $\mathrm{d}^{\frac{1}{2}}: I \rightarrow \mathbb{k}$ satisfying $\mathrm{d}^{\frac{1}{2}}\left(i^{*}\right)=\mathrm{d}^{\frac{1}{2}}(i)$ and $\mathrm{d}(i)=\mathrm{d}^{\frac{1}{2}}(i)^{2}$ for all $i \in I$. Define the operator

$$
\delta: H \rightarrow H \quad \text { given by } \delta=\bigoplus_{i j k} \mathrm{~d}^{\frac{1}{2}}(k) \operatorname{Id}_{H_{i j}^{k}} \oplus \mathrm{d}^{\frac{1}{2}}(k) \operatorname{Id}_{H_{k}^{i j}} .
$$

Let $\delta^{A}=A \delta A$ and $\delta^{B}=B \delta B$.

Lemma $19 \delta, \delta^{A}, \delta^{B}$ are symmetric commuting invertible operators satisfying:

$$
\begin{aligned}
& S \delta=\delta S, \quad S \delta^{A}=\delta^{B} S, \\
& A \delta^{B}=\delta^{B} A, \quad B \delta^{A}=\delta^{A} B, \\
& A \delta=\delta^{A} A, \quad B \delta=\delta^{B} B, \\
& L=\left(\delta / \delta^{A}\right)^{2}, \quad R=\left(\delta / \delta^{B}\right)^{2}, \\
& T \delta_{1}=T \delta_{4}, \quad T \delta_{1}^{B}=T \delta_{2}, \quad T \delta_{1}^{A}=T \delta_{3}^{A}, \\
& T \delta_{2}^{A}=T \delta_{3}^{B}, \quad T \delta_{2}^{B}=T \delta_{4}^{B}, \quad T \delta_{3}=T \delta_{4}^{A} \text {. }
\end{aligned}
$$


Proof First,

$$
\delta, \quad \delta^{A}=\bigoplus_{i j k} \mathrm{~d}^{\frac{1}{2}}(i) \mathrm{Id}_{H_{i j}^{k} \oplus H_{k}^{i j}} \quad \text { and } \quad \delta^{B}=\bigoplus_{i j k} \mathrm{~d}^{\frac{1}{2}}(j) \operatorname{Id}_{H_{i j}^{k} \oplus H_{k}^{i j}}
$$

obviously commute. Notice that on $H_{i j}^{k} \oplus H_{k^{*}}^{j^{*} i^{*}}, \delta$ acts by the scalar $\mathrm{d}^{\frac{1}{2}}(k)=\mathrm{d}^{\frac{1}{2}}\left(k^{*}\right)$. As $S$ stabilizes these summands of $H, S$ and $\delta$ commute which is the first equality of (19). Similarly, the other equality in (19) and Equations (20), (21) are easily deduced from the relation satisfied by $A, B, S$ and the definitions of $\delta, \delta^{A}, \delta^{B}$.

We will now show that the equalities in (22) are a consequence of the fact that $(A, d)$ is a t-ambi pair. Consider the isomorphisms

$$
\begin{aligned}
H_{k}^{i j} \rightarrow H^{i j k^{*}} & =H\left(i, j, k^{*}\right), & & y \mapsto\left(y \otimes w_{k^{*}}^{-1}\right) b_{V_{k}}, \\
H_{i j}^{k} \rightarrow H^{k j^{*} i^{*}} & =H\left(k, j^{*}, i^{*}\right), & & x \mapsto\left(x \otimes w_{j^{*}}^{-1} \otimes w_{i^{*}}^{-1}\right)\left(\operatorname{Id}_{V_{i}} \otimes b_{V_{j}} \otimes \operatorname{Id}_{V_{i}^{*}}\right) b_{V_{i}} .
\end{aligned}
$$

Using these isomorphisms the pairings $(,)_{i j k}: H(i, j, k) \otimes_{\mathbb{k}} H\left(k^{*}, j^{*}, i^{*}\right) \rightarrow \mathbb{k}$ and $\langle\rangle:, H \otimes H \rightarrow \mathbb{k}$ given in Equations (5) and (11), respectively, are related by:

$$
(*, *)_{i, j, k^{*}}=\mathrm{d}\left(V_{k}\right)\langle *, *\rangle_{\mid H_{k}^{i j} \otimes H_{i j}^{k}}=\langle\delta(*), \delta(*)\rangle_{\mid H_{k}^{i j} \otimes H_{i j}^{k}}
$$

Remark that the isomorphisms of (25) commute with $A$ and $B$ and so we have

$$
\begin{aligned}
\mathrm{d}\left(V_{k}\right)\langle x, L y\rangle_{\mid H_{k j *}^{i} \otimes H_{i}^{k j^{*}}} & =\mathrm{d}\left(V_{k}\right)\langle A x, A y\rangle_{\mid H_{k}^{i j} \otimes H_{i j}^{k}} \\
& =(x, y)_{i, j, k^{*}}=\mathrm{d}\left(V_{j}\right)\langle x, y\rangle_{\mid H_{i^{*} k}^{j} \otimes H_{j}^{i * k}}, \\
\mathrm{~d}\left(V_{k}\right)\langle x, R y\rangle_{\mid H_{k j^{*}}^{i} \otimes H_{i}^{k j^{*}}} & =\mathrm{d}\left(V_{k}\right)\langle B x, B y\rangle_{\mid H_{k}^{i j} \otimes H_{i j}^{k}} \\
& =(x, y)_{i, j, k^{*}}=\mathrm{d}\left(V_{i}\right)\langle x, y\rangle_{\mid H_{k j *}^{i} \otimes H_{i}^{k j^{*}}} .
\end{aligned}
$$

Thus, $L y=\delta^{2}\left(\delta^{A}\right)^{-2}(y), R=\delta^{2}\left(\delta^{B}\right)^{-2}(y)$ and this implies Equation (22).

The form $T$ on $H^{\otimes 4}$ is 0 on most of its summands. In particular, it can only be nonzero if the morphisms are composable which mean that the summand has the form

$$
H_{k l}^{m} \otimes_{\mathbb{k}} H_{i j}^{k} \otimes_{\mathbb{k}} H_{n}^{j l} \otimes_{\mathbb{k}} H_{m}^{i n}
$$

for some $(i, j, k, l, m, n) \in I^{6}$. Equations (23), (24) follow from the fact that on these summand the operators $\delta, \delta^{A}, \delta^{B}$ act by easily identifiable scalars. For example, on the summand of (27) the operators $\delta_{1}^{B}$ and $\delta_{2}$ act as

$$
\delta_{\mid H_{k l}^{m}}^{B}=\mathrm{d}^{\frac{1}{2}}(k) \mathrm{Id} \quad \text { and } \quad \delta_{\mid H_{i j}^{k}}=\mathrm{d}^{\frac{1}{2}}(k) \mathrm{Id},
$$

respectively. 
Theorem 20 The $\Psi$-system of Proposition 18 extends to a $\hat{\Psi}$-system where

$$
R^{\frac{1}{2}}=\frac{\delta}{\delta^{B}} \quad \text { and } \quad C^{\frac{1}{2}}=\mathrm{Id} .
$$

In this case, $L^{\frac{1}{2}}=\frac{\delta}{\delta^{A}}$.

Proof The Equations (6a) and (6b) are trivial except for $B R^{\frac{1}{2}} B=R^{-\frac{1}{2}}$ which is a consequence of the fact that conjugation by $B$ exchange $\delta$ and $\delta^{B}$ (see (21)). Equations (6c) and (6d) follow from Equations (23) and (24). For example,

$$
T R_{1}^{\frac{1}{2}} L_{2}^{\frac{1}{2}}=T \frac{\delta_{1} \delta_{2}}{\delta_{1}^{B} \delta_{2}^{A}}=T \frac{\delta_{1}}{\delta_{2}^{A}} \quad \text { and } \quad T R_{3}^{\frac{1}{2}} L_{4}^{\frac{1}{2}}=T \frac{\delta_{3} \delta_{4}}{\delta_{3}^{B} \delta_{4}^{A}}=T \frac{\delta_{4}}{\delta_{3}^{B}}
$$

are equal. Finally,

$$
L^{\frac{1}{2}}=B A R^{-\frac{1}{2}} A B=B A \frac{\delta^{B}}{\delta} A B=B \frac{\delta^{B}}{\delta^{A}} A A B=\frac{\delta}{\delta^{A}} B A A B=\frac{\delta}{\delta^{A}} .
$$

Theorem 21 Let $L$ be a nonempty link in a closed connected orientable 3-manifold $M$ (as above we regard $L$ as a graph with no vertices). Let $\rho \in \mathcal{M}(M, \mathcal{G})$ and $\zeta \in$ $H^{1}(M ; \mathbb{Z} / 2 \mathbb{Z})$. Let $T V(M, L, \rho)$ be the invariant arising from the relative $\mathcal{G}$-spherical structure of $\mathcal{C}$ and let $\mathrm{K}(M, L, \rho, \zeta)$ be the invariant arising from the $\hat{\Psi}$-system described above. Then $\operatorname{TV}(M, L, \rho)=\mathrm{K}(M, L, \rho, \zeta)$. In particular, $\mathrm{K}(M, L, \rho, \zeta)$ is a well-defined complex number which is independent of the choice of the cohomology class $\zeta$ and the square root $\mathrm{d}^{\frac{1}{2}}$ of $\mathrm{d}$.

Proof First, using Lemma 19 and Theorem 20 it is easy to compute the operator $q: H \rightarrow H:$

$$
q=R^{\frac{1}{2}} A R^{-\frac{1}{2}} A L^{-\frac{1}{2}} C^{-\frac{1}{2}}=\frac{\delta}{\delta^{B}} A \frac{\delta^{B}}{\delta} A \frac{\delta^{A}}{\delta}=\frac{\delta}{\delta^{B}} \frac{\delta^{B}}{\delta^{A}} \frac{\delta^{A}}{\delta}=\mathrm{Id}_{H} .
$$

Remark that as $q$ is responsible for the ambiguity of $\tilde{q}$ in $\mathrm{K}$, we see that in our context, this ambiguity disappears.

From duality and the equality $\mathrm{d}(m) \operatorname{Id}_{H_{k l}^{m}}=\left.\delta^{2}\right|_{H_{k l}^{m}}$, we have symmetrized $T$-forms $T_{s}, \bar{T}_{s}: H^{\otimes 4} \rightarrow \mathbb{k}$ given by $T_{s}=T \delta_{1}^{2}=T \delta_{4}^{2}$ and $\bar{T}_{s}=\bar{T} \delta_{1}^{2}=\bar{T} \delta_{4}^{2}$. Let

$$
h=H_{k l}^{m} \otimes_{\mathbb{k}} H_{i j}^{k} \otimes_{\mathbb{k}} H_{n}^{j l} \otimes_{\mathbb{k}} H_{m}^{i n} \quad \text { and } \quad h^{\prime}=H_{i n}^{m} \otimes H_{j l}^{n} \otimes H_{k}^{i j} \otimes H_{m}^{k l} .
$$

After identifying the modules $H_{k}^{i j}$ and $H_{j^{*} i^{*}}^{k^{*}}$ and with the $H\left(i, j, k^{*}\right.$ ) (using the isomorphisms in Equation (25)) we have that

$$
\left.\bar{T}_{s}\right|_{h^{\prime}}=\left.\mathrm{d}(m) \bar{T}\right|_{h^{\prime}}=\left|\begin{array}{ccc}
i & j & k \\
l & m & n
\end{array}\right|,\left.\quad T_{s}\right|_{h}=\left.\mathrm{d}(m) T\right|_{h}=\left|\begin{array}{lll}
n & j^{*} & l \\
k & m & i
\end{array}\right| .
$$


Note the $6 j$-symbol in the last equality correspond to the same spherical graph with opposite orientation.

Let $(\mathcal{T}, \mathcal{L})$ be an $H$-triangulation of $(M, L)$. Let $\Phi$ be an admissible $\mathcal{G}$-coloring of $\mathcal{T}$ such that $[\Phi]=\rho \in \mathcal{M}(M, \mathcal{G})$. Let $c$ be a integral charge on $(\mathcal{T}, \mathcal{L})$ such that $[c]=\zeta \in H^{1}(M ; \mathbb{Z} / 2 \mathbb{Z})$. For each state $\varphi$ of $\Phi$ let us write $T V_{\varphi}\left(\right.$ resp. $\left.\mathrm{K}_{\varphi}\right)$ for the summand of $T V(M, L, \rho)$ (resp. $\mathrm{K}(M, L, \rho, \zeta))$ corresponding to $\varphi$. In other words, we have

$$
T V(M, L, \rho)=\sum_{\varphi \in \mathrm{St}(\Phi)} T V_{\varphi}, \quad \mathrm{K}(M, L, \rho, \zeta)=\sum_{\varphi \in \mathrm{St}(\Phi)} \mathrm{K}_{\varphi}
$$

Let $\varphi$ be a state of $\Phi$. We will show that $T V_{\varphi}=\mathrm{K}_{\varphi}$. First, notice that the factors of $T V_{\varphi}$ and $\mathrm{K}_{\varphi}$ corresponding to $\mathrm{b}$ are identical. Let $t$ be a tetrahedron of $\mathcal{T}$ with ordered vertices $v_{1}, v_{2}, v_{3}, v_{4}$. We will now compute the vector $|t|_{\varphi}^{c}$ which is used in the construction of $\mathrm{K}$. Let $a=c\left(v_{1} v_{2}\right), b=c\left(v_{2} v_{3}\right)$ and $i, j, k, l, m, n$ be the indices given in Equation (16). By definition we have

$$
T(a, b)=T q_{1}^{4 a b} R_{1}^{b} R_{2}^{-a} L_{3}^{-a} R_{3}^{-b}=T\left(\frac{\delta_{1}}{\delta_{1}^{B}}\right)^{2 b}\left(\frac{\delta_{2}}{\delta_{2}^{B}}\right)^{-2 a}\left(\frac{\delta_{3}}{\delta_{3}^{A}}\right)^{-2 a}\left(\frac{\delta_{3}}{\delta_{3}^{B}}\right)^{-2 b} .
$$

Using Lemma 19 and the fact that $\delta, \delta^{A}$ and $\delta^{B}$ all act as scalars on a multiplicity space we have

$$
\left.T(a, b)\right|_{h}=\left.\left(S_{1}^{t} S_{2}^{t}\right) T_{s}\right|_{h}
$$

where

$$
\begin{aligned}
& S_{1}^{t}=(\mathrm{d}(i) \mathrm{d}(l))^{a}(\mathrm{~d}(j) \mathrm{d}(m))^{b}(\mathrm{~d}(k) \mathrm{d}(n))^{c\left(v_{1} v_{3}\right)}, \\
& S_{2}^{t}=\left(\mathrm{d}^{\frac{1}{2}}(m) \mathrm{d}^{\frac{1}{2}}(k) \mathrm{d}^{\frac{1}{2}}(n) \mathrm{d}^{\frac{1}{2}}(m)\right)^{-1} .
\end{aligned}
$$

Similarly, $\left.\bar{T}(a, b)\right|_{h^{\prime}}=\left.\left(S_{1} S_{2}\right) \bar{T}_{s}\right|_{h^{\prime}}$. If $t$ is right oriented then $|t|_{\varphi}^{c}$ is the vector in the module on right-hand side of Equation (17) corresponding to $\left.T(a, b)\right|_{h}$. Similarly, if $t$ is left oriented then $|t|_{\varphi}^{c}$ is the vector in $H_{m}^{i n} \otimes_{\mathbb{k}} H_{n}^{j l} \otimes_{\mathbb{k}} H_{i j}^{k} \otimes_{\mathbb{k}} H_{k l}^{m}$ corresponding to $\left.\bar{T}(a, b)\right|_{h^{\prime}}$. Thus, from Equation (28) we have $|t|_{\varphi}^{c}=S_{1}^{t} S_{2}^{t}|t|_{\varphi}$, where $|t|_{\varphi}$ is the tensor used in the construction of $T V$.

Now for each tetrahedron $t$ of $\mathcal{T}$ we have a scalar $S_{1}^{t}$ as described above. Each of these scalars is the product $\prod_{e} \mathrm{~d}(\varphi(e))^{c(e)}$, where $e$ runs over the 6 edges of $t$. Let $e$ be an edge of $\mathcal{T}$. The portion of $\prod_{t \in \mathcal{T}_{3}} S_{1}^{t}$ corresponding to the edge $e$ is

$$
\prod_{e^{\prime} \in \epsilon_{\mathcal{T}}^{-1}(e)} \mathrm{d}\left(\varphi\left(e^{\prime}\right)\right)^{c\left(e^{\prime}\right)}=\mathrm{d}(\varphi(e))^{\sum_{e^{\prime}} c\left(e^{\prime}\right)}=\mathrm{d}(\varphi(e))^{c_{e}},
$$


where $c_{e}=0$ if $e$ belongs to $\mathcal{L}$ and $c_{e}=1$ otherwise (for notation see Section 3.5). Thus, $\prod_{t \in \mathcal{T}_{3}} S_{1}^{t}=\prod_{e \in \mathcal{T} \backslash \mathcal{L}} \mathrm{d}(\varphi(e))$.

For each $t \in \mathcal{T}_{3}$, the contribution of $S_{2}^{t}$ can be considered as the operator $\otimes_{f} \delta_{f}^{-1}$, where $f$ runs over the set of the 4 faces of $t$ and $\delta_{f}$ is $\delta$ applied to the multiplicity module $H$ associated to the face $f$. This factor is exactly what is needed to relate the pairs $(,)_{i j k}$ and $\langle$,$\rangle ; see Equation (26). Combining all the statements above we see$ that $T V_{\varphi}=\mathrm{K}_{\varphi}$ and so the invariants are equal.

\section{Relative homotopy quantum field theory}

In this section we will extend the invariant $T V$ of 3-manifolds with triangulated boundary to an invariant of 3-manifolds bounding surfaces with dots. We will also prove the main topological theorems of the paper.

\subsection{A $(2+1)$-cobordism category $\mathcal{C o b}^{\Delta}$ of triangulated surfaces}

The first step in extending $T V$ is the introduction of an "oscillating path" in a triangulated surface.

An oscillating path in a triangulated surface $\Sigma$ is a map $\eta$ : edges of $\Sigma\} \rightarrow\{-1,0,1\}$ that satisfies the following conditions: Let $P=\eta^{-1}(\{-1,1\})$ be the support of $\eta$ and $|P|$ be the 1-dimensional simplicial complex which is the closure of $P$. Then we require that any connected component of $|P|$ is homeomorphic to a segment and contains an even number of edges. We also require that $\eta$ takes alternatively values +1 and -1 on the sequence of edges of any of these paths.

We can represent an oscillating path on a triangulation by coloring some of its edges with + and - (see Figure 4).

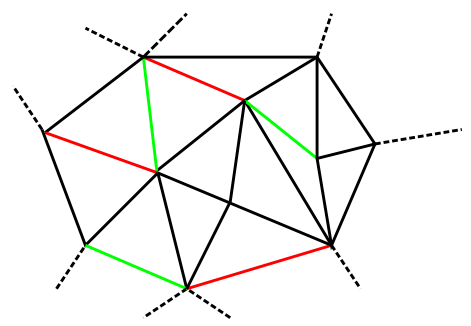

Figure 4: A triangulated surface with an oscillating path: $\eta($ edge $)=\eta$ (color) with $\eta($ red $)=+1, \eta($ green $)=-1, \eta($ black $)=0$ 
We define the number of strands of $(\Sigma, \eta)$ to be the cardinal of the set of vertices $\Sigma_{0}$ of $\Sigma$ minus the number of edges in the support of $\eta$. This is the number of connected components of $\Sigma_{0} \cup|P|$.

Let $\Sigma_{1}$ and $\Sigma_{2}$ be two triangulated oriented surfaces with admissible $\mathcal{G}$-colorings and oscillating paths. Let $\Sigma_{2}^{*}$ be the surface $\Sigma_{2}$ with opposite orientation. An enriched cobordism from $\Sigma_{1}$ to $\Sigma_{2}$ is a quadruplet $(M, f, Y, \rho)$ where:

(i) $M$ is an oriented compact 3-manifold.

(ii) $f: \Sigma_{1} \sqcup \Sigma_{2}^{*} \rightarrow \partial M$ is a diffeomorphism.

(iii) $Y \subset M$ is a graph in $M$ rooted in the triangulation $\mathcal{T}^{\partial}$ of $\partial M$ induced from $\Sigma_{1} \sqcup \Sigma_{2}^{*}$ under $f$ such that if $e$ is an edge of $\Sigma_{1}$ (resp. $\Sigma_{2}$ ) whose value under the oscillating path is +1 (resp. -1 ) then there exits a connected component $Y_{e}$ of $Y$ and a trivializing disk $D^{2}$ embedded in $M$ with $\operatorname{Int}\left(D^{2}\right) \cap Y=\varnothing$ and $\partial D^{2}=e \cup Y_{e}$ (see Figure 5).

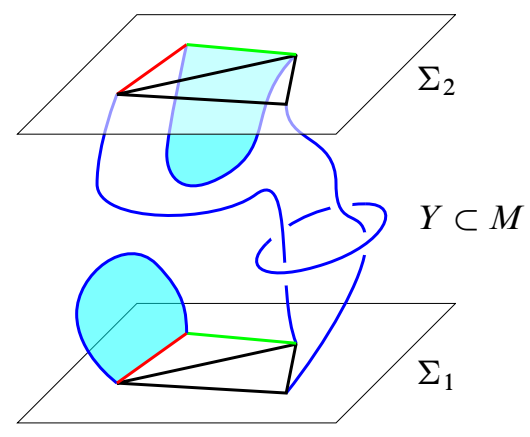

Figure 5: A cobordism between triangulated surfaces with oscillating paths, here the trivializing disks are the shaded regions

(iv) $\rho$ is an admissible representation of $\left(M, \mathcal{T}^{\partial}, Y\right)$ that restricts to both of the admissible $\mathcal{G}$-colorings of $\Sigma_{2}$ and $\Sigma_{1}$.

The triplet $(M, Y, \rho)$ is regarded up to diffeomorphism that is trivial on the boundary (where we use $f$ to identify $\partial M$ and $\Sigma_{1} \sqcup \Sigma_{2}^{*}$ ).

One can glue an enriched cobordism $(M, f, Y, \rho)$ from $\Sigma_{1}$ to $\Sigma_{2}$ with an enriched cobordism $\left(M^{\prime}, f^{\prime}, Y^{\prime}, \rho^{\prime}\right)$ from $\Sigma_{2}$ to $\Sigma_{3}$. The result is an enriched cobordism $\left(M^{\prime \prime}, f^{\prime \prime}, Y^{\prime \prime}, \rho^{\prime \prime}\right)$ from $\Sigma_{1}$ to $\Sigma_{3}$, where $Y^{\prime \prime}$ is the union of the graphs $Y$ and $Y^{\prime}$. The set of vertices of $Y^{\prime \prime}$ is $Y_{0}^{\prime \prime}=\left(Y_{0} \cup Y_{0}^{\prime}\right) \backslash Z$, where $Y_{0}$ and $Y_{0}^{\prime}$ are the vertices of $Y$ and $Y^{\prime}$, respectively and $Z=Y_{0} \cap Y_{0}^{\prime}$ is the subset of these vertices that are on $\Sigma_{2} \hookrightarrow M^{\prime \prime}$. Remark that van Kampen's Theorem for groupoids (see [5]) implies that 
$\pi\left(M^{\prime \prime}, Y_{0} \cup Y_{0}^{\prime}\right)$ is the pushout of the maps $\pi\left(\Sigma_{2}, Z\right) \rightarrow \pi\left(M, Y_{0}\right)$ and $\pi\left(\Sigma_{2}, Z\right) \rightarrow$ $\pi\left(M^{\prime}, Y_{0}^{\prime}\right)$. Thus, there exists an unique representation $\tilde{\rho}$ of $\left(M^{\prime \prime}, Y_{0} \cup Y_{0}^{\prime}\right)$ which restrict to both $\rho$ and $\rho^{\prime}$. The representation $\rho^{\prime \prime}$ is the restriction of $\tilde{\rho}$ to $\left(M^{\prime \prime}, Y_{0}^{\prime \prime}\right)$. Loosely speaking, a path $\gamma$ in $\pi\left(M^{\prime \prime}, Y_{0}^{\prime \prime}\right)$ can be cut to a finite composition of smaller paths in $\pi\left(M, Y_{0}\right)$ and $\pi\left(M, Y_{0}^{\prime}\right)$, and $\rho^{\prime \prime}(\gamma)$ is the product of the images by $\rho$ and $\rho^{\prime}$ of these smaller paths.

Theorem 22 There is a monoidal category $\mathcal{C}_{0}{ }^{\Delta}$ whose objects are quadruplets $(\Sigma, \mathcal{T}, \eta, \Phi)$ formed by an oriented surface $\Sigma$ with a quasiregular triangulation $\mathcal{T}$, an oscillating path $\eta$ and an admissible $\mathcal{G}$-coloring $\Phi$ of $\mathcal{T}$, and whose morphisms are enriched cobordisms. The tensor product is given by disjoint union and the composition by gluing along the boundaries.

Proof It is well known that cobordisms of surfaces form a monoidal category. It is easy to adapt the proof of this result to enriched cobordisms. The only nontrivial point is to prove that objects have an identity morphism. Let us construct the identity morphism $M=(\Sigma \times[0,1], Y, \rho)$ of $(\Sigma, \mathcal{T}, \eta, \Phi)$ as follows. The underlying 3-manifold is the cylinder $\Sigma \times[0,1]$. The graph $Y$ is a tangle as in the schematic picture of Figure 6 .

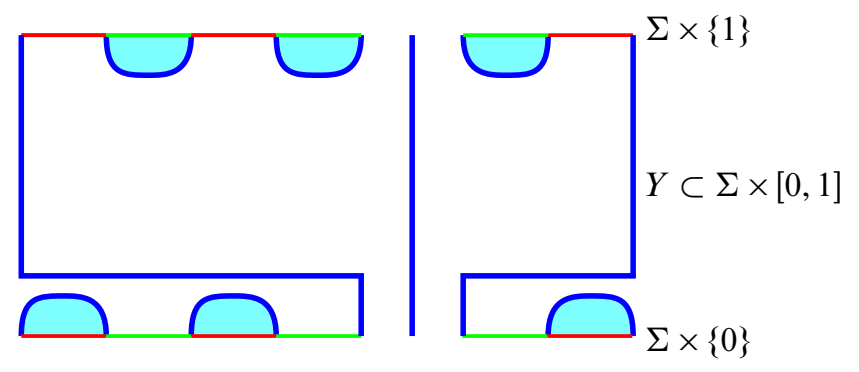

Figure 6: The identity cobordism for a surface $\Sigma$ with 3 strands

$Y$ is the unique tangle (up to isotopy) which is vertical except in a neighborhood of $\Sigma \times\{0\}$ and $\Sigma \times\{1\}$ where it is contained in $|P| \times[0,1]$ (recall $|P|$ is the union of edges $e$ with $\eta(e)= \pm 1)$. The representation $\rho$ is the pull back of $\Phi$ by the projection $p: \Sigma \times[0,1] \rightarrow \Sigma$. One can check that gluing an enriched cobordism $M^{\prime}$ with $M$ along $\Sigma$ does not change the isomorphism class of $M^{\prime}$.

Let $\widetilde{\Sigma}=(\Sigma, \mathcal{T}, \eta, \Phi)$ be an object of $\mathcal{C} o b^{\Delta}$. The dual object $\widetilde{\Sigma}^{*}$ of $\widetilde{\Sigma}$ in $\mathcal{C} o b^{\Delta}$ is defined as follows. Let $\Sigma^{*}$ be the surface $\Sigma$ with opposite orientation. Let $\mathcal{T}^{*}=\mathcal{T}$ be the triangulation of $\Sigma^{*}$ and let $\Phi^{*}=\Phi$ be the $\mathcal{G}$-coloring of $\mathcal{T}^{*}$. Finally, let $\eta^{*}=-\eta$ be the oscillating path of $\mathcal{T}^{*}$. We define $\widetilde{\Sigma}^{*}=\left(\Sigma^{*}, \mathcal{T}^{*}, \eta^{*}, \Phi^{*}\right)$. 
Let $\Gamma$ be the trivalent graph dual to $\Sigma$. As in Equation (14) we can define $H(\widetilde{\Sigma})=$ $\bigoplus_{\varphi \in \operatorname{St}(\Phi)} H\left(\Gamma_{\varphi}\right)$. Changing the orientation of $\Sigma$ changes all the indexes of $\Gamma_{\varphi}$ to their dual and so the pairing (10) induces a nondegenerate pairing

$$
(\cdot, \cdot)_{0}: H\left(\widetilde{\Sigma}^{*}\right) \otimes H(\widetilde{\Sigma}) \rightarrow \mathbb{k} .
$$

Consider the operator $\mathrm{d}_{\widetilde{\Sigma}}$ on $H(\widetilde{\Sigma})$ given by

$$
\mathrm{d} \widetilde{\Sigma}=\bigoplus_{\varphi \in \operatorname{St}(\Phi)}\left(\prod_{e \in \mathcal{T}_{1}} \mathrm{~d}(\varphi(e))\right) \operatorname{Id}_{H\left(\Gamma_{\varphi}\right)} .
$$

Define the nondegenerate pairing

$$
(\cdot, \cdot): H\left(\widetilde{\Sigma}^{*}\right) \otimes H(\widetilde{\Sigma}) \rightarrow \mathbb{k}, \quad x \otimes y \mapsto\left(x, \mathrm{~d}_{\widetilde{\Sigma}}(y)_{0}\right)=\left(\mathrm{d}_{\widetilde{\Sigma}^{*}}(x), y\right)_{0} .
$$

Let $\widetilde{\Sigma}^{\prime}=\left(\Sigma^{\prime}, \mathcal{T}^{\prime}, \eta^{\prime}, \Phi^{\prime}\right)$ be another object of $\mathcal{C} o b^{\Delta}$. Since $H\left(\widetilde{\Sigma}_{i}^{*}\right) \cong H\left(\widetilde{\Sigma}_{i}\right)^{*}$, the pairing in (29) induces an isomorphism

$$
H\left(\widetilde{\Sigma}^{*} \sqcup \widetilde{\Sigma}^{\prime}\right)=H\left(\widetilde{\Sigma}^{*}\right) \otimes H\left(\widetilde{\Sigma}^{\prime}\right) \cong \operatorname{Hom}_{\mathbb{k}}\left(H(\widetilde{\Sigma}), H\left(\widetilde{\Sigma}^{\prime}\right)\right) .
$$

If $(M, f, Y, h)$ is an enriched cobordism from $\widetilde{\Sigma}$ to $\widetilde{\Sigma}^{\prime}$ then using (30) we can identify $T V\left(M, \mathcal{T}^{\partial}, Y, h\right)$ with an element of $\operatorname{Hom}_{\mathbb{k}}\left(H(\widetilde{\Sigma}), H\left(\widetilde{\Sigma}^{\prime}\right)\right)$, where $\mathcal{T}^{\partial} \simeq \mathcal{T}^{*} \sqcup \mathcal{T}^{\prime}$ is the triangulation of $\partial M$ induced by $f$.

In [29, Section 2.3] a mapping $\mathcal{C} \rightarrow \mathcal{C}^{\prime}$ of categories is called a semifunctor if it satisfies the first condition of the definition of a functor: namely, it sends the composition of morphisms to the composition of their images. If $\mathcal{C}^{\prime}$ is abelian, a semifunctor leads to an honest functor: to each object of $\mathcal{C}$ assign the coimage in $\mathcal{C}^{\prime}$ of the image by the semifunctor of the identity morphism of this object, then naturally extend this assignment to morphisms. With this in mind, we define the following functor $Q$. The definition of the state sum $T V$ implies that the mapping $\mathcal{C} o b^{\Delta} \rightarrow \mathcal{V}$ ect defined by $\widetilde{\Sigma} \mapsto H(\widetilde{\Sigma})$ and $\left(M, \mathcal{T}^{\partial}, Y, h\right) \mapsto T V\left(M, \mathcal{T}^{\partial}, Y, h\right) \in \operatorname{Hom}_{\mathbb{k}}\left(H(\widetilde{\Sigma}), H\left(\widetilde{\Sigma}^{\prime}\right)\right)$ is a monoidal semifunctor. Thus, we have a functor $Q: \mathcal{C}_{o b} b^{\Delta} \rightarrow$ Vect, where $Q(\widetilde{\Sigma})$ is given by $H(\widetilde{\Sigma})$ modulo the kernel of the image of $\operatorname{Id}_{\widetilde{\Sigma}}$ under this semifunctor.

\subsection{A $(2+1)$-cobordism category $\mathcal{C} o b$ of marked surfaces}

In this subsection, we remove the requirement that surfaces are triangulated.

A marked surface is a closed oriented surface $\Sigma$ with a finite set of points $m \subset \Sigma$ such that $m$ has a nonempty intersection with any connected component of $\Sigma$. The elements of $\mathrm{m}$ are called marks. We consider marked surfaces equipped with a representation in $\mathcal{G}$ that is an element of $\mathcal{M}(\Sigma, \mathrm{m}, \mathcal{G})$. 
If $\Sigma_{1}$ and $\Sigma_{2}$ are two marked surfaces with representations in $\mathcal{G}$, a marked cobordism from $\Sigma_{1}$ to $\Sigma_{2}$ is a quadruplet $(M, f, Y, \rho)$ where:

(i) $M$ is an oriented compact 3-manifold.

(ii) $f: \Sigma_{1} \sqcup \Sigma_{2}^{*} \rightarrow \partial M$ is a diffeomorphism.

(iii) $Y \subset M$ is a graph rooted in the subset $\mathrm{m} \subset \partial M$ formed by the image under $f$ of the marks of $\Sigma_{1}$ and $\Sigma_{2}$.

(iv) $\rho$ is an element of $\mathcal{M}\left(M, Y_{0}, \mathcal{G}\right)$ that restricts to the representations of $\Sigma_{1}$ and $\Sigma_{2}$ in $\mathcal{G}$.

The triplet $(M, Y, \rho)$ is regarded up to diffeomorphism which is trivial with respect to the boundary (where we use $f$ to identify $\Sigma_{1} \sqcup \Sigma_{2}^{*}$ with $\partial M$ ).

Let $\mathcal{C} o b$ be the monoidal category whose objects are marked surfaces with representation in $\mathcal{G}$ and morphisms are marked cobordisms. There is an obvious forgetful semifunctor $\mathcal{C} b^{\Delta} \rightarrow \mathcal{C} o b$ sending the set of vertices of a triangulation to the set of marks. We modify this semifunctor to construct an equivalence of categories. The main point here is that the number of marks must be reduced.

If $(\Sigma, \mathcal{T})$ is a triangulated surface with an oscillating path $\eta$, we can associate a set of marks $\mathrm{m}$ containing one point in each connected component of the support $|P|$ of $\eta$ as follows: $\mathrm{m}$ is the set of vertices $v$ of $\mathcal{T}$ such that for any edge $e$ containing $v$, $\eta(e) \geq 0$. Hence the number of marks of $(\Sigma, \mathcal{T}, \eta)$ is equal to the number of strands of $(\Sigma, \eta)$. Restricting the representation in $\mathcal{G}$ of an object $\widetilde{\Sigma}$ of $\mathcal{C} o b^{\Delta}$, we get an object $\mathcal{E}(\widetilde{\Sigma})$ of $\mathcal{C} o b$. In order to extends this map to a functor $\mathcal{E}: \mathcal{C} o b^{\Delta} \rightarrow \mathcal{C} o b$ we need to define some special cobordisms.

Let $\widetilde{\Sigma}=(\Sigma, \mathcal{T}, \eta, \Phi)$ be an object of $\mathcal{C} o b^{\Delta}$ and let $\bar{\Sigma}=\mathcal{E}(\widetilde{\Sigma})$. Consider the enriched cobordism $\Sigma \times[0,1]$ that represents the identity of $\widetilde{\Sigma}$ (see Figure 6). Using the forgetful semifunctor $\mathcal{C} o b^{\Delta} \rightarrow \mathcal{C} o b$ we can view this enriched cobordism as marked cobordism of $\left(\Sigma, \mathcal{T}_{0}, \Phi\right)$. Cutting this marked cobordism along $\Sigma \times\left\{\frac{1}{2}\right\}$, we can see it as the composition of two morphisms

$$
\mathrm{C}_{\tilde{\Sigma}}^{\bar{\Sigma}}:\left(\Sigma, \mathcal{T}_{0}, \Phi\right) \rightarrow \bar{\Sigma} \quad \text { and } \quad \mathrm{C} \frac{\widetilde{\Sigma}}{\Sigma}: \bar{\Sigma} \rightarrow\left(\Sigma, \mathcal{T}_{0}, \Phi\right)
$$

in $\mathcal{C} o b$ such that

$$
\mathrm{C}_{\tilde{\Sigma}}^{\bar{\Sigma}} \circ \mathrm{C}_{\bar{\Sigma}}^{\tilde{\Sigma}}=\mathrm{Id}_{\bar{\Sigma}}
$$

and $\mathrm{C}_{\bar{\Sigma}} \widetilde{\widetilde{\Sigma}} \circ \mathrm{C}_{\widetilde{\Sigma}}^{\bar{\Sigma}}$ is the image of $\operatorname{Id}_{\widetilde{\Sigma}}$ under the forgetful semifunctor $\mathcal{C} o b^{\Delta} \rightarrow \mathcal{C} o b$. 
We define $\mathcal{E}$ on morphisms as follows. Let $\widetilde{\Sigma}^{\prime}$ be another object of $\mathcal{C} o b^{\Delta}$ and let $\bar{\Sigma}^{\prime}=\mathcal{E}\left(\widetilde{\Sigma}^{\prime}\right)$. For $M \in \operatorname{Hom}_{\mathcal{C} b^{\Delta}}\left(\tilde{\Sigma}, \widetilde{\Sigma}^{\prime}\right)$ define

$$
\mathcal{E}(M)=\mathrm{C}_{\widetilde{\Sigma}^{\prime}}^{\bar{\Sigma}^{\prime}} \circ M \circ \mathrm{C}_{\bar{\Sigma}} \in \operatorname{Hom}_{\mathcal{C} o b}\left(\bar{\Sigma}, \bar{\Sigma}^{\prime}\right)
$$

Lemma 23 The functor $\mathcal{E}: \mathcal{C} o b^{\Delta} \rightarrow \mathcal{C} o b$ is an equivalence of monoidal categories.

Proof We need to show that $\mathcal{E}$ is a full, faithful and essentially surjective. Consider the map $\operatorname{Hom}_{\mathcal{C o b}^{\Delta}}\left(\widetilde{\Sigma}, \widetilde{\Sigma}^{\prime}\right) \rightarrow \operatorname{Hom}_{\mathcal{C o b}}\left(\mathcal{E}(\widetilde{\Sigma}), \mathcal{E}\left(\widetilde{\Sigma}^{\prime}\right)\right)$ induced by $\mathcal{E}$. This map is invertible with inverse

$$
\operatorname{Hom}_{\mathcal{C} o b}\left(\mathcal{E}(\widetilde{\Sigma}), \mathcal{E}\left(\widetilde{\Sigma}^{\prime}\right)\right) \rightarrow \operatorname{Hom}_{\mathcal{C} o b^{\Delta}}\left(\widetilde{\Sigma}, \widetilde{\Sigma}^{\prime}\right) \quad \text { given by } \bar{M} \mapsto \mathrm{C} \frac{\widetilde{\Sigma}_{\bar{\Sigma}^{\prime}}^{\prime}}{\circ} \bar{M} \circ \mathrm{C} \widetilde{\Sigma}_{\widetilde{\Sigma}}^{\overline{\bar{s}}}
$$

Therefore, $\mathcal{E}$ is full and faithful. The functor $\mathcal{E}$ is essentially surjective because any marked surface has a quasiregular triangulation $\mathcal{T}$ with oscillating path containing those vertices of $\mathcal{T}$ which are not in $\mathrm{m}$.

In fact, if two objects of $\mathcal{C}_{o b}{ }^{\Delta}$ have the same underlying marked surface $\bar{\Sigma}$, then they are canonically isomorphic (using the cylinders of Lemma 23) and thus we can define $\bar{Q}(\bar{\Sigma})$ as the result of the identification of these spaces along these isomorphisms. This construction naturally extends to a functor

$$
\bar{Q}: \mathcal{C} o b \rightarrow \mathcal{V e c t}
$$

We call this functor a relative quantum field theory.

\subsection{Proofs of Theorems 14, 15 and 16}

Throughout this section, we keep notation of Theorem 16 . We begin by explaining that any two $H$-triangulations of $\left(M, \mathcal{T}^{\prime}, Y\right)$ can be related by elementary moves adding or removing vertices, edges, etc. We call an elementary move positive if it adds edges and negative if it removes edges.

The elementary moves (see [17]) are the so-called $H$-bubble moves (Figure 7(a)), the H-Pachner $2 \leftrightarrow 3$ moves (Figure 7(b)) and the H-lune moves (Figure 7(c)).

It is understood that a negative move is only allowed if it is the inverse of a positive move (hence these moves do not change the graph). The $H$-lune move may be expanded as a composition of $H$-bubble moves and $H$-Pachner moves (see [2, Section 2.1]), but it will be convenient for us to use the $H$-lune moves directly. 


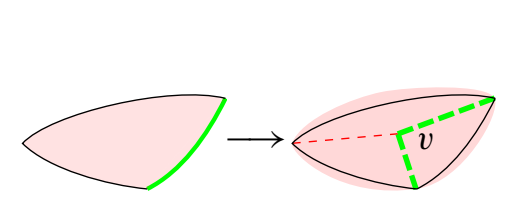

(a) $H$-bubble move

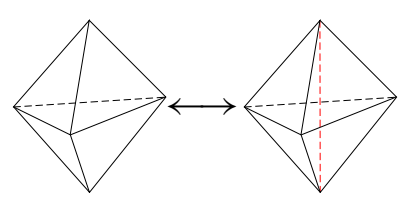

(b) H-Pachner move

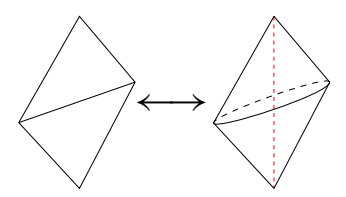

(c) H-lune move

Figure 7: Elementary moves

Proof of Theorem 14 As in the statement of the theorem, let $\mathcal{T}^{\partial}$ be a quasiregular triangulation of $\partial M$ and let $Y$ be a graph in $M$ rooted in $\mathcal{T}^{\partial}$.

In the proof, we shall use the language of skeletons of 3-manifolds dual to the language of triangulations (see, for instance, $[27 ; 2]$ ). A skeleton of $M$ is a 2-dimensional polyhedron $P$ in $M$ such that $M \backslash P$ is a disjoint union of open (half) 3-balls and locally $P$ looks like a plane, or a union of 3 half-planes with common boundary line in $\mathbb{R}^{3}$, or a cone over the 1 -skeleton of a tetrahedron. A typical skeleton of $M$ is constructed from a triangulation $\mathcal{T}$ of $M$ by taking the union $P_{\mathcal{T}}$ of the 2-cells dual to its edges. This construction establishes a bijective correspondence $\mathcal{T} \leftrightarrow P_{\mathcal{T}}$ between the quasiregular triangulations $\mathcal{T}$ of $M$ and "quasiregular" skeletons $P$ of $M$ such that every 2-face of $P$ is a disk adjacent to two distinct components of $M-P$. If $(\mathcal{T}, \mathcal{Y})$ is an $H$-triangulation of $(M, Y)$, to specify the graph $\mathcal{Y}$ in dual skeleton we provide some faces of $P_{\mathcal{T}}$ with dots such that each component of $M-P_{\mathcal{T}}$ is adjacent to at least two (distinct) dotted faces. These dots correspond to the intersections of $\mathcal{Y}$ with the 2-faces. Here the vertices $Y_{0}$ of $Y$ become a set of distinguished components of $M \backslash P_{\mathcal{T}}$. The elementary moves on the $H$-triangulations may be translated to this dual language and give the well-known Matveev-Piergallini moves on skeletons adjusted to the setting of Hamiltonian graphs; see [2].

Take a collar spine $S^{\prime}$ of $\left(M^{\prime}, \Gamma\right)$ as in [29, Theorems 6.4.A \& B], where $M^{\prime}=$ $M \backslash V(Y)$ for an open tubular neighborhood $V(Y)$ of $Y$ and $\Gamma$ is a graph in the boundary of $M^{\prime}$ which is the disjoint union of the trivalent graph dual to the triangulation of $\partial M$ union one meridian circle for each edge (and circle component) of $Y$. Then we fill the meridian circle of $S^{\prime}$ with a dotted disk and get a skeleton $S$ of $M$ in the complement of which a (half) 3-ball has at least one region in its boundary with a dot. Taking the dual of $S$ we obtain an $H$-triangulation $(\mathcal{T}, \mathcal{Y})$ of $(M, Y)$ relative to 
$\mathcal{T}^{\partial}$ which may not be quasiregular. If $\mathcal{T}$ is not quasiregular then $S$ contains a region which has the same (half) 3-cell on both sides or a region with both sides in $Y_{0}$. We call such a region bad.

The skeleton $S$ can be modified by the Matveev-Piergallini moves dual to the elementary moves to obtain a skeleton which is dual to a quasiregular $H$-triangulation. In the case when $M$ is closed this was done by Baseilhac and Benedetti [2, proof of Proposition 4.20]. In particular, for a 3-cell with a bad region they do a dual $H$-bubble move on $S$ which introduces a new 3-cell which is partially bounded by a two cell which they call a capping disk. Then they use dual $H$-Pachner moves to slide a portion of the capping disk over the bad region, thus dividing a (half) 3-cell with the bad region into two 3-cell (or a 3-cell and a half 3-cell) with no bad regions. The proof of [2] can be applied in the case when $M$ has a boundary and $Y$ is a graph.

Proposition 24 Let $M$ be a compact 3-manifold, $\mathcal{T}^{\partial}$ a quasiregular triangulation of $\partial M$ and $Y$ a graph in $M$ rooted in $\mathcal{T}^{\partial}$. Any two $H$-triangulations of $(M, Y)$ relative to $\mathcal{T}^{\partial}$ can be related by a finite sequence of $H$-bubble moves and $H$-Pachner moves in the class of $H$-triangulations of $(M, Y)$ relative to $\mathcal{T}^{\partial}$.

Proof Let $\left(\mathcal{T}_{1}, \mathcal{Y}_{1}\right)$ and $\left(\mathcal{T}_{2}, \mathcal{Y}_{2}\right)$ be $H$-triangulation of $(M, Y)$ relative to $\mathcal{T}^{\partial}$. By doing positive $H$-bubble moves if necessary we can assume that each edge of $Y$ is realized by the same number of edges in both $\mathcal{Y}_{1}$ and $\mathcal{Y}_{2}$. Let $P_{T_{i}}$ be the skeleton dual to $\mathcal{T}_{i}$ where each edge of $\mathcal{Y}_{i}$ corresponds to a dotted face as in the proof of Theorem 14. Replacing a neighborhood of each of these dots with a circle we obtain a collar spine $S_{i}$ of $\left(M \backslash V(Y), \Gamma_{i}\right)$, where $\Gamma_{i}$ is the graph in the boundary of $M \backslash V(Y)$ formed by these circles. The assumption on the edges of $\mathcal{Y}_{1}$ and $\mathcal{Y}_{2}$ implies that $\Gamma_{1}$ and $\Gamma_{2}$ are isotopic. Then [29, Theorem 6.4.B] implies that two spines $S_{1}$ and $S_{2}$ are related by a sequence of dual $H$-Pachner and $H$-lune moves. In [2, Proposition 4.23], Baseilhac and Benedetti prove that by adding and sliding capping disks this sequence of moves leads to a sequence of moves in the class of $H$-triangulations connecting $(\mathcal{T}, \mathcal{Y})$ and $\left(\mathcal{T}^{\prime}, \mathcal{Y}^{\prime}\right)$

Lemma 25 Let $\Phi$ be an admissible $\mathcal{G}$-coloring of $\mathcal{T}$. Suppose that $\left(\mathcal{T}^{\prime}, \mathcal{Y}^{\prime}\right)$ is an $H$-triangulation obtained from $(\mathcal{T}, \mathcal{Y})$ by a negative $H$-Pachner, $H$-lune or $H$-bubble move. Then $\Phi$ restricts to an admissible $\mathcal{G}$-coloring $\Phi^{\prime}$ of $\mathcal{T}^{\prime}$ and

$$
\operatorname{TV}(\mathcal{T}, \mathcal{Y}, \Phi)=T V\left(\mathcal{T}^{\prime}, \mathcal{Y}^{\prime}, \Phi^{\prime}\right)
$$

Proof The proof is exactly the same as the one of [17, Lemma 18]. That is we can translate the $H$-Pachner, $H$-lune and $H$-bubble moves into algebraic identities: the Biedenharn-Elliott identity, the orthonormality relation and (13), respectively. 
Let $(\mathcal{T}, \mathcal{Y})$ be a $H$-triangulation of $(M, Y)$. A $\mathcal{G}$-gauge of $\mathcal{T}$ is a map from the set of vertices $\mathcal{T}_{0}$ of $\mathcal{T}$ to $\mathcal{G}$. The $\mathcal{G}$-gauges of $\mathcal{T}$ form a multiplicative group which acts on the set of $\mathcal{G}$-colorings of $\mathcal{T}$ as follows. If $\delta$ is a $\mathcal{G}$-gauge of $\mathcal{T}$ and $\Phi$ is a $\mathcal{G}$-coloring of $\mathcal{T}$, then the $\mathcal{G}$-coloring $\delta . \Phi$ is given by

$$
(\delta . \Phi)(e)=\delta\left(v_{e}^{-}\right) \Phi(e) \delta\left(v_{e}^{+}\right)^{-1},
$$

where $v_{e}^{-}$(resp. $v_{e}^{+}$) is the initial (resp. terminal) vertex of an oriented edge $e$. Let $\mathcal{T}_{0}^{i}$ be the set of vertices of $\mathcal{T}$ that are not vertices of $\mathcal{Y}$ so $\mathcal{T}_{0}=Y_{0} \sqcup \mathcal{T}_{0}^{i}$. Let $\mathcal{G}^{i}$ be the set of $\mathcal{G}$-gauges $\delta: \mathcal{T}_{0} \rightarrow \mathcal{G}$ such that $\delta(v)=1$ if $v \in Y_{0}$.

Proof of Theorem 15 Take any $\mathcal{G}$-coloring $\Phi$ of $\mathcal{T}$ which restricts to $\rho$. We say that a vertex $v$ of $\mathcal{T} \backslash Y_{0}$ is bad for $\Phi$ if there is an oriented edge $e$ in $\mathcal{T}$ outgoing from $v$ such that $\Phi(e) \in \mathcal{X}$. Since $(\mathcal{T}, \mathcal{Y})$ is an $H$-triangulation any edge not in $\partial M$ has at least one endpoint not in $Y_{0}$. Thus, it is clear that $\Phi$ is admissible if and only if $\Phi$ has no bad vertices. We show how to modify $\Phi$ to reduce the number of bad vertices. Let $v$ be a bad vertex for $\Phi$ and let $E_{v}$ be the set of all oriented edges of $\mathcal{T}$ outgoing from $v$. Pick any

$$
g \in \mathcal{G} \backslash\left(\bigcup_{e \in E_{v}}\left(\mathcal{X} \Phi(e)^{-1}\right)\right) .
$$

Let $\delta^{v, g}$ to be the $\mathcal{G}$-valued gauge defined by

$$
\delta^{v, g}\left(v^{\prime}\right)= \begin{cases}g & \text { if } v=v^{\prime} \\ 1 & \text { else. }\end{cases}
$$

Then $\delta^{v, g} . \Phi$ takes values in $\mathcal{G} \backslash \mathcal{X}$ on all edges of $\mathcal{T}$ incident to $v$ and takes the same values as $\Phi$ on all edges of $\mathcal{T}$ not incident to $v$. Here we use the fact that the edges of $\mathcal{T}$ are not loops which follows from the quasiregularity of $\mathcal{T}$. The transformation $\Phi \mapsto \delta^{v, g} . \Phi$ decreases the number of bad vertices. Repeating this argument, we find a $\mathcal{G}$-coloring without bad vertices which restricts to $\rho$.

Remark that for $\Phi \in \mathcal{M}\left(M, \mathcal{T}_{0}, \mathcal{G}\right)$, and for $g \in \mathcal{G}^{\mathcal{T}_{0}^{i}}$, the $\mathcal{G}$-colorings $\Phi$ and $\delta_{g} . \Phi$ restrict to the same representation in $\mathcal{M}\left(M, Y_{0}, \mathcal{G}\right)$.

Lemma 26 If $\Phi_{1}$ and $\Phi_{2}$ in $\mathcal{M}\left(M, \mathcal{T}_{0}, \mathcal{G}\right)$ restrict to the same representation $\rho$ in $\mathcal{M}\left(M, Y_{0}, \mathcal{G}\right)$, then there exists an element $g \in \mathcal{G}^{i}$ with $\delta_{g} \cdot \Phi_{1}=\Phi_{2}$.

Proof We assume in the proof, without loss of generality, that $M$ is connected. When $M$ is closed and $Y_{0}$ is empty, the elements of $\mathcal{M}(M, \mathcal{G})$ bijectively correspond to the $\mathcal{G}$-colorings of $\mathcal{T}$ considered up to gauge transformations (see for example [26]). Therefore, assume $Y_{0}$ is not empty. 
We first distinguish a set of edge $\overline{\mathcal{Y}}$. Let start with $\overline{\mathcal{Y}}=\mathcal{Y}$. Suppose that some vertex of $\mathcal{T}$ is not connected to $Y_{0}$ by a path in $\overline{\mathcal{Y}}$. We say that such a vertex is isolated. Then there exists an edge of $\mathcal{T}$ whose ends are an isolated vertex on one side and a vertex not isolated in the other side. We then add this edge to $\overline{\mathcal{Y}}$. We repeat this process until there is no more isolated vertex. One can check that he had added one edge of $\mathcal{T}$ for each circle component of $\mathcal{Y}$. A path of length $n$ is a sequence of $n$ oriented edges $e_{1}, \ldots, e_{n}$ where the target of $e_{i}$ is equal to the source of $e_{i+1}$. Choose an orientation of the edges of $Y$. This orientation can be extended to a compatible orientation of the edges of $\overline{\mathcal{Y}}$ such that for every vertex $v$ of $\mathcal{T}$ there exists an unique shortest path $\gamma_{v}$ in $\overline{\mathcal{Y}}$ from some vertex of $Y_{0}$ to $v$. To be more precise, recall that every oriented edge of $Y$ is realized by a path in $\mathcal{Y}$. So there is an unique compatible orientation of the edges of $\mathcal{Y}$. As for the added edges of $\overline{\mathcal{Y}} \backslash \mathcal{Y}$, we choose the orientation toward the originally isolated vertex. The union of paths $\gamma_{v}$ where $v$ is a vertex of $\mathcal{T}$ is a disjoint union of trees rooted in $Y_{0}$. We say a vertex $v$ of $\mathcal{T}$ is $\operatorname{good}$ if $\Phi_{1}(e)=\Phi_{2}(e)$ for every edge $e$ of $\gamma_{v}$. In particular, every vertex of $Y_{0}$ is good.

Suppose all vertices of $\mathcal{T}$ are good. Then $\Phi_{1}=\Phi_{2}$ because for any oriented edge $e$ of $\mathcal{T}$ from $v_{1}$ to $v_{2}$, we have

$$
\Phi_{1}(e)=\Phi_{1}\left(\gamma_{v_{1}}\right)^{-1} \rho\left(\gamma_{v_{1}} e \gamma_{v_{2}}^{-1}\right) \Phi_{1}\left(\gamma_{v_{2}}\right)=\Phi_{2}\left(\gamma_{v_{1}}\right)^{-1} \rho\left(\gamma_{v_{1}} e \gamma_{v_{2}}^{-1}\right) \Phi_{2}\left(\gamma_{v_{2}}\right)=\Phi_{2}(e)
$$

Thus, it remains to show that we can modify $\Phi_{1}$ by a gauge transformation to increase the number of good vertices. Let $e$ be an edge of $\overline{\mathcal{Y}}$ from $v_{1}$ to $v_{2}$, where $v_{1}$ is good and $v_{2}$ is not good. Then $\gamma_{v_{2}}=\gamma v_{1} e$ and so $\delta^{v_{2}, g} . \Phi_{1}(e)=\Phi_{2}(e)$, where $g=\Phi_{2}(e)^{-1} \Phi_{1}(e)$. If $v$ is a good vertex the path $\gamma_{v}$ can not pass through $v_{2}$ as $\Phi_{1}(e) \neq \Phi_{2}(e)$. Therefore, the set of good vertices strictly increases when one replaces $\Phi_{1}$ with $\delta^{v_{2}, g} . \Phi_{1}$.

Lemma 27 Let $v_{0} \in \mathcal{T}_{0}^{i}$ and $c: \mathcal{T}_{0} \rightarrow \mathcal{G}$ be a map such that $c(v)=1$ for all $v \neq v_{0}$ and $c\left(v_{0}\right) \notin \mathcal{X}$. If $\Phi$ and $\delta_{c} . \Phi$ are admissible $\mathcal{G}$-colorings of $\mathcal{T}$, then $\operatorname{TV}(\mathcal{T}, \mathcal{Y}, \Phi)=$ $\operatorname{TV}\left(\mathcal{T}, \mathcal{Y}, \delta_{c} . \Phi\right)$

Proof A similar claim is proved in [17, Lemma 27] when $\mathcal{G}$ is a commutative group. Since $v_{0}$ is in $\mathcal{T}_{0}^{i}$ the vertex $v_{0}$ is the endpoint for exactly two edges $e_{1}, e_{2}$ of $Y$. As shown in [17, proof of Lemma 27] there is a sequence of elementary moves through quasiregular triangulation with admissible colorings from $(\mathcal{T}, \mathcal{Y}, \Phi)$ to $\left(\mathcal{T}, \mathcal{Y}, \delta_{c} . \Phi\right)$. This sequence starts with a positive $H$-bubble move at $e_{1}$ (where we introduce the color $\left.c\left(v_{0}\right)\right)$ and ends with a negative $H$-bubble move at $e_{2}$. 
Lemma 28 If $\Phi$ and $\Phi^{\prime}$ are two admissible $\mathcal{G}$-colorings of $\mathcal{T}$ that restrict to the same representation $\rho \in \mathcal{M}\left(M, Y_{0}, \mathcal{G}\right)$, then $\operatorname{TV}(\mathcal{T}, \mathcal{Y}, \Phi)=T V\left(\mathcal{T}, \mathcal{Y}, \Phi^{\prime}\right)$.

Proof Since $\Phi$ and $\Phi^{\prime}$ represent the same element of $\mathcal{M}\left(M, Y_{0}, \mathcal{G}\right)$ by Lemma 26 there exists pairs $\left(v_{i}, g_{i}\right) \in\{$ vertices of $\mathcal{T}\} \times \mathcal{G}$ such that

$$
\Phi^{\prime}=\delta^{v_{n}, g_{n}} \delta^{v_{n-1}, g_{n-1}} \cdots \delta^{v_{1}, g_{1}} . \Phi,
$$

where $\delta^{v_{i}, g_{i}}$ is the $\mathcal{G}$-valued gauge, defined as in (33), which takes nontrivial values at a single vertex $v_{i}$, for all $i=1, \ldots, n$. We prove the desired equality by induction on $n$. If $n=0$ then $\Phi^{\prime}=\Phi$ and the equality is clear. Otherwise, let $E_{1}$ be the set of (oriented) edges of $\mathcal{T}$ beginning at $v_{1}$. Pick any

$$
g \in \mathcal{G} \backslash\left[\mathcal{X} \cup \bigcup_{e \in E_{1}}\left(\mathcal{X} \Phi(e)^{-1}\right) \cup \bigcup_{e \in E_{1}}\left(\mathcal{X} \Phi^{\prime}(e)^{-1} g_{1}\right)\right] .
$$

Then $\delta^{v_{1}, g} . \Phi$ and $\delta^{v_{n}, g_{n}} \delta^{v_{n-1}, g_{n-1}} \cdots \delta^{v_{2}, g_{2}} \delta^{v_{1}, g} . \Phi=\delta^{v_{1}, g g_{1}^{-1}} . \Phi^{\prime}$ are admissible colorings. Lemma 27 and the induction assumption imply that

$$
\begin{aligned}
\operatorname{TV}(\mathcal{T}, \mathcal{L}, \Phi, c) & =\operatorname{TV}\left(\mathcal{T}, \mathcal{L}, \delta^{v_{1}, g} \cdot \Phi, c\right) \\
& =\operatorname{TV}\left(\mathcal{T}, \mathcal{L}, \delta^{v_{n}, g_{n}} \delta^{v_{n-1}, g_{n-1}} \ldots \delta^{v_{2}, g_{2}} \delta^{v_{1}, g} . \Phi, c\right) \\
& =\operatorname{TV}\left(\mathcal{T}, \mathcal{L}, \Phi^{\prime}, c\right) .
\end{aligned}
$$

Theorem 29 Let $(\mathcal{T}, \mathcal{Y})$ and $\left(\mathcal{T}^{\prime}, \mathcal{Y}^{\prime}\right)$ be two $H$-triangulations of $(M, Y)$ relative to $\mathcal{T}^{\partial}$ such that $\left(\mathcal{T}^{\prime}, \mathcal{Y}^{\prime}\right)$ is obtained from $(\mathcal{T}, \mathcal{Y})$ by a single $H$-Pachner move, $H-$ bubble move, or $H$-lune move. Then for any admissible $\mathcal{G}$-colorings $\Phi, \Phi^{\prime}$ of $\mathcal{T}, \mathcal{T}^{\prime}$, respectively, restricting to the same element of $\mathcal{M}\left(M, Y_{0}, \mathcal{G}\right)$, we have

$$
T V(\mathcal{T}, \mathcal{Y}, \Phi)=T V\left(\mathcal{T}^{\prime}, \mathcal{Y}^{\prime}, \Phi^{\prime}\right)
$$

Proof For concreteness, assume that $\mathcal{T}^{\prime}$ is obtained from $\mathcal{T}$ by a negative move. The admissible $\mathcal{G}$-coloring $\Phi$ of $\mathcal{T}$ restricts to an admissible $\mathcal{G}$-coloring $\Phi^{\prime \prime}$ of $\mathcal{T}^{\prime}$ which restrict to the same element of $\mathcal{M}\left(M, Y_{0}, \mathcal{G}\right)$. Now Lemma 25 implies that $\operatorname{TV}(\mathcal{T}, \mathcal{Y}, \Phi)=T V\left(\mathcal{T}^{\prime}, \mathcal{Y}^{\prime}, \Phi^{\prime \prime}\right)$ and Lemma 28 implies that $T V\left(\mathcal{T}^{\prime}, \mathcal{Y}^{\prime}, \Phi^{\prime \prime}\right)=$ $T V\left(\mathcal{T}^{\prime}, \mathcal{Y}^{\prime}, \Phi^{\prime}\right)$.

Proof of Theorem 16 From Proposition 24 we know that any two $H$-triangulation of $(M, Y)$ are related by a finite sequence of elementary moves. Then the result follows from the Theorem 29 by induction on the number of moves. 


\section{Quantum groups at roots of unity}

In this section we first recall some of the deep results established by De Concini, Kac, Procesi, Reshetikhin and Rosso in the series of papers [8], [9], [10] and [11]. We then prove that the modules studied in these papers give rise to the topological invariants described in Sections 2, 3 and 4. In particular, in Sections 5.1 and 5.2 we give definitions and properties of the algebras $\mathcal{U}$ and $\mathcal{U}^{H}$ associated to a simple Lie algebra $\mathfrak{g}$. In Section 5.3 we discuss the category of weight modules over these algebras. Since the constructions in the first three subsections are some what abstract, in Section 5.4 we give a concrete example when $\mathfrak{g}=\mathfrak{s l}_{2}$. Then we give descriptions of the pivotal structure, braiding and modified dimensions. The section is concluded with a theorem stating that the category of finite-dimensional weight modules over $\mathcal{U}$ is a relative $\mathcal{G}$-spherical category.

\subsection{The quantum groups $\mathcal{U}$ and $\mathcal{U}^{H}$}

Let $\mathfrak{g}$ be a simple finite-dimensional complex Lie algebra of rank $n$ and dimension $2 N+n$ with a root system. Fix a set of simple roots $\left\{\alpha_{1}, \ldots, \alpha_{n}\right\}$ and let $\Delta^{+}$be the corresponding set of positive roots. Also, let $A=\left(a_{i j}\right)_{1 \leq i, j \leq n}$ be the Cartan matrix corresponding to these simple roots. There exists a diagonal matrix $D=$ $\operatorname{diag}\left(d_{1}, \ldots, d_{n}\right)$ such that $D A$ is symmetric and positive-definite. Let $\mathfrak{h}$ be the Cartan subalgebra of $\mathfrak{g}$ generated by the vectors $H_{1}, \ldots, H_{n}$, where $H_{j}$ is determined by $\alpha_{i}\left(H_{j}\right)=a_{j i}$. Let $L_{R}$ be the root lattice which is the $\mathbb{Z}$-lattice generated by the simple roots $\left\{\alpha_{i}\right\}$. Let $\langle$,$\rangle be the form on L_{R}$ given by $\left\langle\alpha_{i}, \alpha_{j}\right\rangle=d_{i} a_{i j}$. Let $L_{W}$ be the weight lattice which is the $\mathbb{Z}$-lattice generated by the elements of $\mathfrak{h}^{*}$ which are dual to the elements $H_{i}, i=1, \ldots, n$. Let $\rho=\frac{1}{2} \sum_{\alpha \in \Delta^{+}} \alpha \in L_{W}$.

Let $r$ be an odd integer such that $r \geq 3$ (and $r \notin 3 \mathbb{Z}$ if $\mathfrak{g}=G_{2}$ ). Let $q=\mathrm{e}^{2 i \pi / r}$ and for $i=1, \ldots, n$, let $q_{i}=q^{d_{i}}$. For $x \in \mathbb{C}$ and $k, l \in \mathbb{N}$ we use the notation:

$$
\begin{gathered}
q^{x}=\mathrm{e}^{\frac{2 i \pi x}{r}}, \quad\{x\}_{q}=q^{x}-q^{-x}, \quad[x]_{q}=\frac{\{x\}_{q}}{\{1\}_{q}}, \\
{[k]_{q}=[1]_{q}[2]_{q} \cdots[k]_{q}, \quad\left[\begin{array}{c}
k \\
l
\end{array}\right]_{q}=\frac{[k]_{q} !}{[l]_{q} ![k-l]_{q} !} .}
\end{gathered}
$$

Remark for $x \in \mathbb{C},\{x\}_{q}=0$ if and only if $x \in \frac{r}{2} \mathbb{Z}$.

Next we consider two quantum groups associated to $\mathfrak{g}$. The Drinfeld-Jimbo quantum group $\mathcal{U}$ is defined as the algebra with generators $K_{\beta}, X_{i}, X_{-i}$ for $\beta \in L_{W}, i=1, \ldots, n$ 
and relations

$$
\begin{aligned}
& K_{0}=1, \quad K_{\beta} K_{\gamma}=K_{\beta+\gamma}, \quad K_{\beta} X_{\sigma i} K_{-\beta}=q^{\sigma\left\langle\beta, \alpha_{i}\right\rangle} X_{\sigma i}, \\
& {\left[X_{i}, X_{-j}\right]=\delta_{i j} \frac{K_{\alpha_{i}}-K_{\alpha_{i}}^{-1}}{q_{i}-q_{i}^{-1}},} \\
& \sum_{k=0}^{1-a_{i j}}(-1)^{k}\left[\begin{array}{c}
1-a_{i j} \\
k
\end{array}\right]_{q_{i}} X_{\sigma i}^{k} X_{\sigma j} X_{\sigma i}^{1-a_{i j}-k}=0, \quad \text { if } i \neq j,
\end{aligned}
$$

where $\sigma= \pm 1$. The algebra $\mathcal{U}$ is a Hopf algebra with coproduct $\Delta$, counit $\epsilon$ and antipode $S$ defined by

$$
\begin{aligned}
& \Delta\left(X_{i}\right)=1 \otimes X_{i}+X_{i} \otimes K_{\alpha_{i}}, \quad S\left(X_{i}\right)=-X_{i} K_{\alpha_{i}}^{-1}, \quad \epsilon\left(X_{i}\right)=\epsilon\left(X_{-i}\right)=0, \\
& \Delta\left(X_{-i}\right)=K_{\alpha_{i}}^{-1} \otimes X_{-i}+X_{-i} \otimes 1, \quad S\left(X_{-i}\right)=-K_{\alpha_{i}} X_{-i}, \quad \epsilon\left(K_{\alpha_{i}}\right)=1 . \\
& \Delta\left(K_{\beta}\right)=K_{\beta} \otimes K_{\beta}, \quad S\left(K_{\beta}\right)=K_{-\beta},
\end{aligned}
$$

The unrolled quantum group $\mathcal{U}^{H}$ is the algebra generated by $K_{\beta}, X_{i}, X_{-i}, H_{i}$ for $\beta \in L_{W}, i=1, \ldots, n$ with relations (34), (35), (36) plus the relations

$$
\left[H_{i}, X_{\epsilon j}\right]=\sigma a_{i j} X_{\sigma j}, \quad\left[H_{i}, H_{j}\right]=\left[H_{i}, K_{\beta}\right]=0,
$$

where $\sigma= \pm 1$. The algebra $\mathcal{U}^{H}$ is a Hopf algebra with coproduct $\Delta$, counit $\epsilon$ and antipode $S$ defined as above on $K_{\beta}, X_{i}, X_{-i}$ and defined on the elements $H_{i}$ for $i=1, \ldots, n$ by

$$
\Delta\left(H_{i}\right)=1 \otimes H_{i}+H_{i} \otimes 1, \quad \epsilon\left(H_{i}\right)=0, \quad S\left(H_{i}\right)=-H_{i} .
$$

As a vector space $\mathcal{U}^{H}$ is isomorphic to $\mathcal{U}_{\mathfrak{h}} \otimes_{\mathbb{C}} \mathcal{U}$ where $\mathcal{U}_{\mathfrak{h}}=\mathbb{C}[\mathfrak{h}]$ is the symmetric algebra of $\mathfrak{h} \otimes_{\mathbb{Z}} \mathbb{C}$. The obvious map $\mathcal{U} \rightarrow \mathcal{U}^{H}$ is an injective morphism of Hopf algebra. Using this morphism we can identify $\mathcal{U}$ with a Hopf subalgebra of $\mathcal{U}^{H}$.

\subsection{The PBW basis}

Let $\alpha_{1}, \ldots, \alpha_{n}$ be any ordering of the simple positive roots and let $s_{i_{1}} s_{i_{2}} \cdots s_{i_{N}}$ be a reduced decomposition of the longest element of the Weyl group. Then $\beta_{1}=\alpha_{i_{1}}$, $\beta_{2}=s_{i_{1}} \alpha_{i_{2}}, \ldots, \beta_{N}=s_{i_{1}} s_{i_{2}} \cdots s_{i_{N-1}} \alpha_{i_{N}}$ is a total ordering of the set of positive roots $\Delta^{+}$. We call $\beta_{*}=\left(\beta_{1}, \ldots, \beta_{N}\right)$ a convex order of $\Delta^{+}$. For each $i=1, \ldots, N$ let $X_{ \pm \beta_{i}}$ be the positive (resp. negative) root vector of $\mathcal{U}$ (see for example [6, Section 8.1 and 9.1]). We say that $\left(X_{ \pm \beta}\right)_{\beta \in \beta_{*}}$ is a convex set of root vectors which depends of the order of $\alpha_{1}, \ldots, \alpha_{n}$ and $s_{i_{1}}, s_{i_{2}}, \ldots, s_{i_{N}}$. 
If $y_{1}, \ldots, y_{m}$ is a set of elements in an algebra then let $\operatorname{Alg}\left\langle y_{i}: i=1, \ldots, m\right\rangle$ and $\operatorname{Span}_{\mathbb{C}}\left\langle y_{i}: i=1, \ldots, m\right\rangle$ be the algebra generated and the complex vector space spanned by these elements, respectively. We will use the following notation (for $\mathcal{U}, \mathcal{U}^{H}, \mathcal{U}_{\mathfrak{h}}$ see Section 5.1):

$$
\begin{aligned}
\mathcal{U}_{+} & =\operatorname{Alg}\left\langle X_{i}: i=1, \ldots, n\right\rangle, \\
\mathcal{U}_{-} & =\operatorname{Alg}\left\langle X_{-i}: i=1, \ldots, n\right\rangle, \\
\mathcal{U}_{++} & =\mathcal{U}_{+} \cap \operatorname{ker} \epsilon, \\
\mathcal{U}_{--} & =\mathcal{U}_{-} \cap \operatorname{ker} \epsilon \\
\mathcal{U}_{+<} & =\operatorname{Span}_{\mathbb{C}}\left\langle X_{\beta_{1}}^{k_{1}} \cdots X_{\beta_{N}}^{k_{N}}: 0 \leq k_{i}<r\right\rangle, \\
\mathcal{U}_{-<} & =\operatorname{Span}_{\mathbb{C}}\left\langle X_{-\beta_{1}}^{k_{1}} \cdots X_{-\beta_{N}}^{k_{N}}: 0 \leq k_{i}<r\right\rangle, \\
\mathcal{Z}^{0} & =\operatorname{Alg}\left\langle K_{\gamma}^{r}, X_{\beta}^{r}: \gamma \in L_{W}, \beta \in \beta_{*}\right\rangle, \\
\mathcal{U}_{0} & =\operatorname{Alg}\left\langle K_{\omega_{i}}:\left\{\omega_{i}\right\}_{i=1, \ldots, n} \text { is the set of fundamental weights }\right\rangle .
\end{aligned}
$$

Remark (1) the algebra $\mathcal{U}_{0}$ is a commutative Hopf subalgebra of $\mathcal{U}$, (2) the spaces $\mathcal{U}_{+<}$and $\mathcal{U}_{-<}$depend on the convex order $\beta_{*}$ of $\Delta^{+}$and (3) it is proven in [9] that $\mathcal{Z}^{0}=\left(\mathcal{Z} \cap \mathcal{U}_{-}\right)\left(\mathcal{Z} \cap \mathcal{U}_{0}\right)\left(\mathcal{Z} \cap \mathcal{U}_{+}\right)$and thus $\mathcal{Z}^{0}$ is independent of $\beta_{*}$.

We will need the following weak version of the PBW Theorem; for a proof see for example $[10 ; 6]$.

Theorem 30 The multiplication map defines vector space isomorphisms

$$
\mathcal{U}_{-} \otimes \mathcal{U}_{0} \otimes \mathcal{U}_{+} \stackrel{\sim}{\longrightarrow} \mathcal{U} \stackrel{\sim}{\sim} \mathcal{U}_{+} \otimes \mathcal{U}_{0} \otimes \mathcal{U}_{-} .
$$

Furthermore, for $\sigma \in\{+,-\}$, the set

$$
\left\{X_{\sigma \beta_{1}}^{k_{1}} X_{\sigma \beta_{2}}^{k_{2}} \cdots X_{\sigma \beta_{N}}^{k_{N}}:\left(k_{1}, \ldots, k_{N}\right) \in \mathbb{N}^{N}\right\}
$$

is a basis of $\mathcal{U}_{\sigma}$ and the set $\left\{K_{\alpha}: \alpha \in L_{W}\right\}$ is a basis of $\mathcal{U}_{0}$.

Note that in Theorem 30 one can reverse the order of the products for the monomials of the basis of $\mathcal{U}_{ \pm}$and still obtain a basis. From [9, Proposition 5.6] we have that $\mathcal{Z}^{0}$ is a Hopf subalgebra of $\mathcal{U}$ contained in the center $\mathcal{U}$.

Let $\overline{\mathcal{G}}$ be the simply connected group associated to $\mathfrak{g}$ and let $T=\exp \mathfrak{h}$ be the maximal torus of $\overline{\mathcal{G}}$. Let $U^{+}$and $U^{-}$be the unipotent radicals of the opposite Borel subgroups $B^{+}$and $B^{-}$, respectively. Recall from [9] that, as a Hopf algebra, $\mathcal{Z}^{0}$ is the coordinate ring of the dual group $\mathcal{G}$ which is the subgroup of $B^{+} \times B^{-}$given by the kernel of the composition $B^{+} \times B^{-} \rightarrow T \times T \rightarrow T$, where $T \times T \rightarrow T$ is the multiplication and $B^{+} \times B^{-} \rightarrow T \times T$ is the quotient modulo the unipotent radical (also see [11, 
Section 6.3]). As a variety, $\mathcal{G}$ can be identified with $U^{-} \times T \times U^{+}$, where the inclusion $U^{-} \times T \times U^{+} \rightarrow B^{+} \times B^{-}$is given by $(u, t, v) \mapsto\left(t u, t^{-1} v\right)$. Thus, $\mathcal{G}$ can be identified with the group of ring homomorphisms $g: \mathcal{Z}^{0} \rightarrow \mathbb{C}$ where the group structure is given by $g h=(g \otimes h) \circ \Delta$. Moreover, $T$ can be identified with the subgroup of $\mathcal{G}$ consisting of elements $g$ which are zero on any element of $\mathcal{Z}^{0} \cap\left(\mathcal{U}_{++} \cup \mathcal{U}_{--}\right)$.

The authors of [11] define a subset of $\mathcal{G}$ called the unramified locus. Let $\mathcal{X}$ be the complement of the unramified locus in $\mathcal{G}$. The set $\mathcal{G} \backslash \mathcal{X}$ is a Zariski dense open subset of $\mathcal{G}$.

We will use the group $\mathcal{G}$ throughout the rest of the paper. In particular, we will show $\mathcal{G}$ gives a grading on a certain category of $\mathcal{U}$-modules where graded pieces corresponding to the unramified locus are semisimple. With this in mind we recall the following theorem.

Theorem $31 \quad[8 ; 9 ; 10 ; 11$, Proposition 5.5] For any $g \in \mathcal{G}$ the following are equivalent:

(i) $\mathcal{U} \otimes_{g: \mathcal{Z}^{0} \rightarrow \mathbb{C}} \mathbb{C}$ is a semisimple algebra.

(ii) $g \notin \mathcal{X}$.

(iii) There are (at least) $r^{n}$ nonisomorphic irreducible $\mathcal{U}$-modules of dimension $r^{N}$ on which any element of $z \in \mathcal{Z}^{0}$ acts by the scalar $g(z)$.

In Corollary 35 we will give a partial description of $\mathcal{X}$.

\subsection{Weight modules}

A multiplicative weight is a $\mathbb{C}$-algebra homomorphism $\kappa: \mathcal{U}_{0} \rightarrow \mathbb{C}$. Given a $\mathcal{U}$-module $V$ and a multiplicative weight $\kappa$, let $E_{\kappa}(V)$ be the weight space consisting of elements $v \in V$ such that any $x \in \mathcal{U}_{0}$ acts on $v$ as the scalar $\kappa(x)$. A $\mathcal{U}$-module $V$ is called a weight module if $V$ splits as a direct sum of its weight spaces and if all elements of $\mathcal{Z}^{0}$ act diagonally on it.

On the other hand, an additive weight is a $\mathbb{C}$-algebra homomorphism $\lambda: \mathcal{U}_{\mathfrak{h}} \rightarrow \mathbb{C}$. Given a $\mathcal{U}^{H}$-module $V$ and an additive weight $\lambda$, let $E_{\lambda}(V)$ be the weight space consisting of elements $v \in V$ such that any $x \in \mathcal{U}_{\mathfrak{h}}$ acts on $v$ as the scalar $\lambda(x)$. A $\mathcal{U}^{H}$-module $V$ is called a weight module if $V$ splits as a direct sum of its weight spaces, all elements of $\mathcal{Z}^{0}$ act diagonally on it, and for any $\beta=\sum_{i} b_{i} \alpha_{i} \in L_{W}$, the element $K_{\beta}$ acts on $V$ as the scalar $\prod_{i} q_{i}^{b_{i} H_{i}}$. An additive weight $\lambda$ induces a multiplicative weight $\kappa=q^{\lambda}$ defined by the rule $\kappa\left(K_{\beta}\right)=\prod_{i} q_{i}^{b_{i} \lambda\left(H_{i}\right)}=q^{\langle\beta, \lambda\rangle}$ for any $\beta=\sum_{i} b_{i} \alpha_{i} \in L_{W}$. If $\gamma$ is a multiplicative or additive weight we call a vector 
$v \in E_{\gamma}(V)$ a weight vector with (multiplicative or additive) weight $\gamma$. The vector space $\mathcal{U}^{H}$ with the adjoint action is a weight module over $\mathcal{U}^{H}$. If $x \in E_{\lambda}\left(\mathcal{U}^{H}\right)$ we say $x$ has weight $\lambda$.

Let $\mathcal{D}$ and $\mathcal{D}^{H}$ be the categories of finite-dimensional weight modules over $\mathcal{U}$ and $\mathcal{U}^{H}$, respectively. Let $\mathcal{D}_{T}$ be the full subcategory of $\mathcal{D}$ whose objects are the modules on which $X_{ \pm \beta}^{r}$ acts by 0 , for all $\beta \in \beta_{*}$. For $g \in \mathcal{G}$, let $\mathcal{D}_{g}$ be the full subcategory of $\mathcal{D}$ whose objects are the modules for which any $x \in \mathcal{Z}^{0}$ acts by the scalar $g(x)$. Then $\mathcal{D}=\bigoplus_{g \in \mathcal{G}} \mathcal{D}_{g}$ is $\mathcal{G}$-graded. Also, if $t \in T \subset \mathcal{G}$ we have that $\mathcal{D}_{t} \subset \mathcal{D}_{T}$ and $\mathcal{D}_{T}=\bigoplus_{t \in T} \mathcal{D}_{t}$

Let $V$ be an object of $\mathcal{D}^{H}$. The first equality in Equation (37) implies that the action of $X_{j}$, for $j=1, \ldots, n$, translates the weight spaces of $V$. Similarly, any $x \in \mathcal{U}_{++} \cup \mathcal{U}_{--}$translate nontrivially the weights. Combining this with the fact that $V$ is finite-dimensional one sees that the operator $x: V \rightarrow V$ is nilpotent. In particular for any $\beta \in \beta_{*}$, the operator $X_{ \pm \beta}^{r}: V \rightarrow V$ is nilpotent and thus the zero operator since $X_{ \pm \beta}^{r} \in \mathcal{Z}^{0}$. By forgetting the action of $\mathcal{U}_{\mathfrak{h}}$ the module $V$ becomes a weight module over $\mathcal{U}$. Moreover, since $X_{ \pm \beta}^{r}: V \rightarrow V$ is the zero operator, $V$ is an object in $\mathcal{D}_{T}$. Thus, we have a forgetful functor

$$
\mathcal{F}: \mathcal{D}^{H} \rightarrow \mathcal{D}_{T}
$$

and we say that objects of $\mathcal{D}_{T}$ and $\mathcal{D}^{H}$ are nilpotent modules.

For any nilpotent module $V$ there exists a vector $v_{ \pm}$such that $X_{ \pm i} v_{ \pm}=0$ for all $i=1, \ldots, n$. Such vectors $v_{+}$and $v_{-}$are called highest weight vectors and lowest weight vectors, respectively. If $V$ is simple then $v_{ \pm}$is unique up to a scalar.

Theorem 32 Let $V \in \mathcal{D}_{g}$ and $V^{\prime} \in \mathcal{D}_{g^{\prime}}$, for $g, g^{\prime} \in \mathcal{G}$. Then:

(i) $\quad V \otimes V^{\prime} \in \mathcal{D}_{g g^{\prime}}$,

(ii) $V^{*} \in \mathcal{D}_{g^{-1}}$,

(iii) $\mathcal{D}_{g}$ is semisimple if and only if $g \notin \mathcal{X}$.

Proof The first two items follows from the fact that $\mathcal{Z}^{0}$ is a Hopf subalgebra of $\mathcal{U}$. The third is a consequence of Theorem 31.

\subsection{Example: $\mathcal{U}=\mathcal{U}\left(\mathfrak{s l}_{2}\right)$ simple weight modules}

To illustrate the different types of modules we use in this paper we give a description of simple $\mathcal{U}$-weight modules when $\mathfrak{g}=\mathfrak{s l}_{2}$; for more details see [6, Chapter 11.1]. For 
general $\mathfrak{g}$, see also [6]. The algebra $\mathcal{U}\left(\mathfrak{s l}_{2}\right)$ has four generators $X_{1}, X_{-1}, k$ and $k^{-1}$. In the notation above, the generator $k$ is $K_{\alpha}$, where $\alpha$ is the fundamental weight.

The group $\mathcal{G}$ can be identified with the group of ring homomorphisms $g: \mathcal{Z}^{0} \rightarrow \mathbb{C}$, where $\mathcal{Z}^{0}=\mathbb{C}\left[k^{ \pm r}, X_{1}^{r}, X_{-1}^{r}\right]$ is the central sub-Hopf algebra. As a Lie group, $\mathcal{G}$ can be embedded in $B^{+} \times B^{-}$via the map

$$
g \mapsto\left(\left(\begin{array}{cc}
g\left(k^{-r}\right) & 0 \\
0 & g\left(k^{r}\right)
\end{array}\right) \times\left(\begin{array}{cc}
1 & g\left(X_{1}^{r}\right) \\
0 & 1
\end{array}\right),\left(\begin{array}{cc}
1 & 0 \\
g\left(X_{-1}^{r}\right) & 1
\end{array}\right) \times\left(\begin{array}{cc}
g\left(k^{r}\right) & 0 \\
0 & g\left(k^{-r}\right)
\end{array}\right)\right) .
$$

It can be shown that $\mathcal{X}$ is the subset of $\mathcal{G}$ defined by the equation

$$
g \in \mathcal{X} \Longleftrightarrow g\left(\left(\{1\}^{2 r} X_{1}^{r} X_{-1}^{r}-k^{2 r}-k^{-2 r}\right)^{2}\right)=4 .
$$

Finally, $T \subset \mathcal{G}$ is the subgroup of elements $t$ such that $t\left(X_{1}^{r}\right)=t\left(X_{-1}^{r}\right)=0$ and $t \in T \cap \mathcal{X}$ if and only if $t\left(k^{4 r}\right)=1$.

A $\mathcal{U}\left(\mathfrak{s l}_{2}\right)$-weight module is finite-dimensional. As we explain now, there are two kinds of simple $\mathcal{U}\left(\mathfrak{s l}_{2}\right)$-weight module: highest weight modules and cyclic modules. Let $V$ be a simple $\mathcal{U}\left(\mathfrak{s l}_{2}\right)$-weight module of degree $g$. In [6], $V$ is called cyclic module if $g\left(X_{1}^{r}\right)$ and $g\left(X_{-1}^{r}\right)$ are nonzero. This terminology emphasis the fact that $X_{+}$and $X_{-}$ permute the weight spaces of $V$ in a cyclic way. A cyclic module has dimension $r$. If $g\left(X_{1}^{r}\right)=g\left(X_{-1}^{r}\right)=0$ then $g \in T$ and $V \in \mathcal{D}_{T}$ is a highest weight module. In this case, $V$ has a unique (up to a scalar) highest weight vector $v_{0}$ such that $k \cdot v_{0}=q^{\lambda / 2} v_{0}$ $(\lambda \in \mathbb{C}$ is unique modulo $2 r \mathbb{Z}$ ). A highest weight module $V$ has dimension $r$ if and only if $2 \lambda \notin\{0,1, \ldots, r-2\}+r \mathbb{Z}$ (see Proposition 34 below). This is in particular true if $g \in T \backslash(\mathcal{X} \cap T)$ that is if $g\left(k^{4 r}\right) \neq 1$.

Remark that Theorem 32 implies that for $g \in \mathcal{G} \backslash \mathcal{X}$, the category $\mathcal{D}_{g}$ has $r$ distinct isomorphic classes of simple modules, all of dimension $r$. Moreover, the modules in $\mathcal{D}_{g}$ are those isomorphic to a finite direct sum of these $r$-dimensional modules. For $g \in \mathcal{X}, \mathcal{D}_{g}$ is not semisimple.

For $t \in T$ and $V \in \mathcal{D}_{t}$ with highest weight $\kappa \in \mathbb{C}^{*}$ as above (ie $k \cdot v_{0}=\kappa v_{0}$ ), a lift of $V$ in $\mathcal{D}^{H}$ consists in choosing a scalar $\lambda \in \mathbb{C}$ by which $H \in \mathcal{U}^{H}$ acts on $v_{0}$ such that $\kappa=q^{\lambda / 2}$. Finally, a module in $\mathcal{D}_{t}^{H}$ has the following alternative description: A module of $\mathcal{D}^{H}$ is in $\mathcal{D}_{t}^{H}$ if and only if all its additive weights are equal to $\lambda$ modulo $2 \mathbb{Z}$ (ie modulo the root lattice).

\subsection{Typical modules}

Let $I^{H}$ be the ideal of $\mathcal{U}^{H}$ generated by the central elements $X_{ \pm \beta}^{r}, \beta \in \beta_{*}$. Let $I=I^{H} \cap \mathcal{U}$ the corresponding ideal in $\mathcal{U}$. The ideals $I$ and $I^{H}$ do not depend on 
the choice of $\beta_{*}$ because they are generated by $\left(\mathcal{Z}^{0} \cap \mathcal{U}_{++}\right) \cup\left(\mathcal{Z}^{0} \cap \mathcal{U}_{--}\right)$. The set of monomials of the PBW basis that contain at least one factor $X_{ \pm \beta}^{k}$, with $k \geq r$, is a basis of $I$. Hence $\mathcal{U}_{ \pm<}$and $I \cap \mathcal{U}_{ \pm}$are complementary vector subspaces of $\mathcal{U}_{ \pm}$(note $\mathcal{U}_{ \pm<}$is defined in Section 5.2). Any nilpotent module is annihilated by $I$.

Let $\kappa \in \operatorname{Hom}_{\mathrm{alg}}\left(\mathcal{U}_{0}, \mathbb{C}\right)$ be a multiplicative weight and consider the one-dimensional $\mathcal{U}_{0} \mathcal{U}_{+}-$module $\left\langle v_{0}\right\rangle$ whose action is determined by $x v_{0}=\kappa(x) v_{0}$ for $x \in \mathcal{U}_{0}$ and $y v_{0}=\epsilon(y) v_{0}$ for $y \in \mathcal{U}_{+}$. This module induces the Verma module $\widetilde{V}_{\kappa}$ which is an infinite-dimensional weight module over $\mathcal{U}$ with highest weight vector $v_{0}$ of multiplicative weight $\kappa$. The module $\widetilde{V}_{\kappa}$ contains a unique maximal submodule not containing $v_{0}$. The quotient of this submodule is an irreducible $\mathcal{U}$-module $V_{\kappa}$ with a highest weight vector of weight $\kappa$. Similarly, an additive weight $\lambda \in \operatorname{Hom}_{\text {alg }}\left(\mathcal{U}_{\mathfrak{h}}, \mathbb{C}\right)$ defines a one-dimensional module over $\mathcal{U}_{\mathfrak{h}} \mathcal{U}_{0} \mathcal{U}_{+}$whose action is given by $\lambda$ on $\mathcal{U}_{\mathfrak{h}}, q^{\lambda}$ on $\mathcal{U}_{0}$ and $\epsilon$ on $\mathcal{U}_{+}$. The same process gives the Verma module $\widetilde{V}_{\lambda}$ with irreducible quotient $V_{\lambda}$, both having highest weight vectors of weight $\lambda$.

If $\lambda \in \operatorname{Hom}_{\mathrm{alg}}\left(\mathcal{U}_{\mathfrak{h}}, \mathbb{C}\right)$ is an additive weight and $\kappa=q^{\lambda} \in \operatorname{Hom}_{\mathrm{alg}}\left(\mathcal{U}_{0}, \mathbb{C}\right)$ is its associated multiplicative weight we denote by $|\lambda|=|\kappa|$ the element of $T \simeq \operatorname{Hom}_{\text {alg }}\left(\mathcal{Z}^{0} \cap \mathcal{U}_{0}, \mathbb{C}\right)$ induced by $\kappa$.

Proposition 33 Let $\lambda$ in $\operatorname{Hom}_{\mathrm{alg}}\left(\mathcal{U}_{\mathfrak{h}}, \mathbb{C}\right)$ be an additive weight and let $\kappa=q^{\lambda}$ in Hom $_{\text {alg }}\left(\mathcal{U}_{0}, \mathbb{C}\right)$ its induced multiplicative weight. Then $V_{\lambda} \in O b\left(\mathcal{D}^{H}\right)$ and $V_{\kappa} \in$ $O b\left(\mathcal{D}_{|\kappa|}\right)$ are the unique (up to isomorphism) irreducible nilpotent module with highest weights $\lambda$ and $\kappa$, respectively. Furthermore, $\mathcal{F}\left(V_{\lambda}\right)$ is isomorphic to $V_{\kappa}$, where $\mathcal{F}$ is the forgetful functor defined in (38).

Proof Let $V$ be the module $V_{\lambda}$ or $V_{\kappa}$ with height weight vector $v_{0}$. Since the ideal $I$ annihilates $V$ we have that $V=\mathcal{U}_{-} v_{0}=\mathcal{U}_{-<v_{0}}$. Therefore, the set

$$
\left\{X_{-\beta_{1}}^{k_{1}} \cdots X_{-\beta_{N}}^{k_{N}} v_{0}: 0 \leq k_{i}<r\right\}
$$

contains a finite basis of weight vectors. The uniqueness of $V$ comes from the universal property of its Verma module $\widetilde{V}: \widetilde{V}$ can be mapped to any module $W$ with a highest weight vector of the same weight as $v_{0}$. If $W$ is irreducible then the kernel of this map is a maximal proper submodule and so $W$ is isomorphic to $V$.

Similarly, the map $\mathcal{F}\left(\widetilde{V}_{\lambda}\right) \stackrel{\sim}{\longrightarrow} \widetilde{V}_{\kappa}$ induces a surjective map $\mathcal{F}\left(V_{\lambda}\right) \rightarrow V_{\kappa}$. If the kernel of this map were nonzero then it would be a nilpotent module with a highest weight vector $w$. But $w$ would also be a highest weight vector of $V_{\lambda}$ generating a proper submodule of $V_{\lambda}$. This is not possible because $V_{\lambda}$ is irreducible. 
Proposition 33 implies that any irreducible nilpotent module in $\mathcal{D}$ is isomorphic to $\mathcal{F}\left(V_{\lambda}\right)$ for some $\lambda$. However, it is not clear that every nilpotent $\mathcal{U}$-module $V$ is isomorphic to $\mathcal{F}(W)$ for some $\mathcal{U}^{H}$ weight module $W$.

Proposition 34 [8, Theorem 3.2] Let $\lambda \in \operatorname{Hom}_{\mathrm{alg}}\left(\mathcal{U}_{\mathfrak{h}}, \mathbb{C}\right)$ be an additive weight and $\kappa=q^{\lambda} \in \operatorname{Hom}_{\mathrm{alg}}\left(\mathcal{U}_{0}, \mathbb{C}\right)$ its induced multiplicative weight. Let $v_{+}$and $v_{-}$be the highest and lowest weight vectors of $V_{\kappa}$, respectively. Then the following are equivalent:

(i) The linear map $\mathcal{U}_{-<} \rightarrow V_{\kappa}$ defined by $x \mapsto x v_{+}$is bijective.

(ii) The linear map $\mathcal{U}_{+<} \rightarrow V_{\kappa}$ defined by $x \mapsto x v_{-}$is bijective.

(iii) $q^{2\langle\lambda+\rho, \beta\rangle+m\langle\beta, \beta\rangle} \neq 1$ for all $\beta \in \Delta^{+}$and $m \in\{1, \ldots r-1\}$.

Proof In [8, Section 3.2], De Concini and Kac define the so called diagonal module $V_{\kappa}^{\prime}=\widetilde{V}_{\kappa} / I \widetilde{V}_{\kappa}$ with highest weight vector of weight $\kappa$. They prove that the third condition is equivalent to the module $V_{\kappa}^{\prime}$ being irreducible (remark they have a misprint with the range of $m$ in their Theorem 3.2). Thus, the proposition follows from the following facts: (1) when $V_{\kappa}^{\prime}$ is irreducible it is isomorphic to $V_{\kappa},(2)$ the maps in the proposition are surjective and $(3) \operatorname{dim}_{\mathbb{C}}\left(\mathcal{U}_{-<}\right)=\operatorname{dim}_{\mathbb{C}}\left(\mathcal{U}_{+<}\right)=\operatorname{dim}_{\mathbb{C}}\left(V_{\kappa}^{\prime}\right)=r^{N}$.

If $\lambda$ and $\kappa=q^{\lambda}$ are weights such that one of the equivalent conditions of Proposition 34 is satisfied then we say that $V_{\lambda}$ and $V_{\kappa}$ are typical modules. When $\mathfrak{g}=\mathfrak{s l}_{2}$ then the typical modules are exactly the simple $r$-dimensional $\mathcal{U}\left(\mathfrak{s l}_{2}\right)$-weight modules described above. The following corollary combined with Proposition 33 implies that any irreducible $\mathcal{U}$-module $V \in \mathcal{D}_{g}$ with $g \in T \backslash \mathcal{X}$ is typical.

Corollary 35 If $g \in T \subset \mathcal{G}$ such that $g\left(K_{\beta}^{r}\right) \neq \pm 1$ for all $\beta \in \Delta^{+}$, then $g \notin \mathcal{X}$.

Proof There exist (nonunique) additive weights $\lambda_{i}$, for $i=1, \ldots, r^{n}$, with induced multiplicative weights $\kappa_{i}$ such that $\left|\kappa_{i}\right|=g$, for all $i=1, \ldots, r^{n}$. Then for all $\beta \in \Delta^{+}$ and $i=1, \ldots, r^{n}$ we have

$$
g\left(K_{\beta}^{r}\right)=\kappa_{i}\left(K_{\beta}^{r}\right)=q^{r\left\langle\lambda_{i}, \beta\right\rangle} .
$$

Combining this with the hypothesis $g\left(K_{\beta}^{r}\right) \neq \pm 1$ we have $q^{2\left\langle\lambda_{i}+\rho, \beta\right\rangle+m\langle\beta, \beta\rangle} \neq 1$ for all $\beta \in \Delta^{+}, m \in\{0, \ldots r-1\}$ and $i \in\left\{1, \ldots, r^{n}\right\}$. Thus, Proposition 34 implies that for each $i=1, \ldots, r^{n}$ the module $V_{\kappa_{i}}$ has dimension $r^{N}$ and the result follows from Theorem 31. 


\subsection{The pivotal structure}

It is well known that $\left\langle 2 \rho, \alpha_{i}\right\rangle=\left\langle\alpha_{i}, \alpha_{i}\right\rangle=2 d_{i}$, where $\alpha_{i}$ is a simple positive root. Then for $\sigma= \pm 1$, Equation (34) implies that $K_{2 \rho} X_{\sigma i} K_{2 \rho}^{-1}=K_{\alpha_{i}} X_{\sigma i} K_{\alpha_{i}}^{-1}$. Combining this fact with $S^{2}\left(X_{\sigma i}\right)=K_{\alpha_{i}} X_{\sigma i} K_{\alpha_{i}}^{-1}$ we have

$$
S^{2}(x)=K_{2 \rho}^{1-r} x K_{2 \rho}^{r-1}
$$

for all $x$ in $\mathcal{U}^{H}$. Equation (39) is a modification (by the central element $K_{2 \rho}^{r}$ ) of a similar well-known equation. We impose this modification to make the modified dimension d "spherical" and the twist invariant by the antipode (see Sections 5.8 and 5.9).

It is a general fact that a category of modules over a Hopf algebra in which the square of the antipode is equal to the conjugation by a group-like element is a pivotal (or sovereign) category (see [4, Proposition 2.9]). Hence, from Equation (39) it follows that $\mathcal{D}$ and $\mathcal{D}^{H}$ are both pivotal $\mathbb{k}$-linear categories with ground ring $\mathbb{C}$. In particular, for any object $V$ in $\mathcal{D}$, the dual object and the duality morphisms are defined as follows: $V^{*}=\operatorname{Hom}_{\mathbb{C}}(V, \mathbb{C})$ and

$$
\begin{array}{ll}
b_{V}: \mathbb{C} \rightarrow V \otimes V^{*} & \text { is given by } 1 \mapsto \sum v_{j} \otimes v_{j}^{*}, \\
d_{V}: V^{*} \otimes V \rightarrow \mathbb{C} & \text { is given by } f \otimes w \mapsto f(w), \\
b_{V}^{\prime}: \mathbb{C} \rightarrow V^{*} \otimes V & \text { is given by } 1 \mapsto \sum v_{j}^{*} \otimes K_{2 \rho}^{r-1} v_{j}, \\
d_{V}^{\prime}: V \otimes V^{*} \rightarrow \mathbb{C} & \text { is given by } v \otimes f \mapsto f\left(K_{2 \rho}^{1-r} v\right),
\end{array}
$$

where $\left\{v_{j}\right\}$ is a basis of $V$ and $\left\{v_{j}^{*}\right\}$ is the dual basis of $V^{*}$.

\subsection{Ambidextrous modules}

Recall the definition of an ambidextrous object given in Section 2. In this subsection we show that typical modules over $\mathcal{U}^{H}$ and $\mathcal{U}$ are ambidextrous. With this in mind we give a general theorem which allows one to prove certain objects are ambidextrous.

We will now assume that $\mathcal{C}$ is a category of $H$-modules for some Hopf $\mathbb{C}$-algebra $H$. If $V$ is any object of $\mathcal{C}$ we have the bilinear pairing $\langle$,$\rangle on V \otimes V^{*}=\left(V \otimes V^{*}\right)^{*}$ given by $\left\langle w, w^{\prime}\right\rangle=d_{V \otimes V^{*}}\left(w \otimes w^{\prime}\right)$. Then for any $f \in \operatorname{End}\left(V \otimes V^{*}\right)$ we have $\left\langle f(w), w^{\prime}\right\rangle=\left\langle w, f^{*}\left(w^{\prime}\right)\right\rangle$.

Theorem 36 Let $V$ be an irreducible $H$-module. Assume there exists $w_{0}, w_{0}^{\prime} \in$ $V \otimes V^{*}$ and $x, y \in H$ such that $\left\langle w_{0}, w_{0}^{\prime}\right\rangle=1$ and $x . w_{0}, y . w_{0}^{\prime}$ are nonzero $H$ invariant vectors (ie any $h \in H$ acts on $x . w_{0}$ and $y . w_{0}^{\prime}$ by the scalar $\varepsilon(h)$ ). Then the module $V$ is ambidextrous if either of the following two conditions hold: 
(a) $\mathbb{C} w_{0}$ and $\mathbb{C} w_{0}^{\prime}$ are invariant lines under the action of $\operatorname{End}_{\mathcal{C}}\left(V \otimes V^{*}\right)$.

(b) $\operatorname{ker} x \subset \operatorname{ker}\left\langle\cdot, w_{0}^{\prime}\right\rangle$ and $\operatorname{ker} y \subset \operatorname{ker}\left\langle w_{0}, \cdot\right\rangle$; here $x, y$ are operators on $V \otimes V^{*}$.

Proof Let $f \in \operatorname{End}_{\mathcal{C}}\left(V \otimes V^{*}\right)$. Since $V$ is simple then any invariant vector of $V \otimes V^{*}$ is proportional to $b_{V}(1)$ because $b_{V}$ generates the one-dimensional vector space $\operatorname{Hom}_{\mathcal{C}}\left(\mathbb{C}, V \otimes V^{*}\right) \simeq \operatorname{Hom}_{\mathcal{C}}(V, V)$. So up to rescaling $x$ and $y$, we may assume that $x \cdot w_{0}=y \cdot w_{0}^{\prime}=b_{V}(1)$. Also, since $f\left(x \cdot w_{0}\right)$ and $f\left(y \cdot w_{0}^{\prime}\right)$ are invariant vectors there exists $\alpha, \alpha^{\prime} \in \mathbb{C}$ with $f\left(x . w_{0}\right)=\alpha x . w_{0}$ and $f\left(y . w_{0}^{\prime}\right)=\alpha^{\prime} y . w_{0}^{\prime}$. Therefore, there exists $\left(v, v^{\prime}\right) \in \operatorname{ker} x \times \operatorname{ker} y$ such that $f\left(w_{0}\right)=\alpha w_{0}+v$ and $f^{*}\left(w_{0}^{\prime}\right)=\alpha^{\prime} w_{0}^{\prime}+v^{\prime}$. Also, $f \circ b_{V}(1)=f\left(x \cdot w_{0}\right)=\alpha x \cdot w_{0}=\alpha b_{V}(1)$ and $f^{*} \circ b_{V}(1)=\alpha^{\prime} b_{V}(1)$.

If Condition (a) holds we can assume $v=v^{\prime}=0$. Then

$$
\alpha=\left\langle f\left(w_{0}\right), w_{0}^{\prime}\right\rangle=\left\langle w_{0}, f^{*}\left(w_{0}^{\prime}\right)\right\rangle=\alpha^{\prime} .
$$

On the other hand, if Condition (b) is satisfied, $\left\langle v, w_{0}^{\prime}\right\rangle=\left\langle w_{0}, v\right\rangle=0$ and so

$$
\alpha=\left\langle f\left(w_{0}\right), w_{0}^{\prime}\right\rangle=\left\langle w_{0}, f^{*}\left(w_{0}^{\prime}\right)\right\rangle=\alpha^{\prime} .
$$

Thus, in both cases we have $f^{*} \circ b_{V}=f \circ b_{V}$.

Lemma 37 Consider the two following elements of $\mathcal{U}$ :

$$
X_{-}=\prod_{\alpha \in \beta_{*}} X_{-\alpha}^{r-1} \quad \text { and } \quad X_{+}=\prod_{\alpha \in \beta_{*}} X_{\alpha}^{r-1} .
$$

If $\gamma_{*}$ is any convex order of $\Delta^{+}$and $\left\{X_{\alpha}\right\}_{\alpha \in \gamma_{*}}$ is the corresponding convex set of root vectors then there exists $a, b \in \mathbb{C}^{*}$ such that

$$
X_{-} \equiv a \prod_{\alpha \in \gamma_{*}} X_{-\alpha}^{r-1} \quad \bmod I \quad \text { and } \quad X_{+} \equiv b \prod_{\alpha \in \gamma_{*}} X_{\alpha}^{r-1} \bmod I .
$$

Proof This is a consequence of (PBW) Theorem 30. Indeed, the weight space of $\mathcal{U}_{+} / I$ with weight $2(r-1) \rho$ is one-dimensional, generated by $\prod_{\alpha \in \gamma_{*}} X_{\alpha}^{r-1}$ for any convex order $\gamma_{*}$ of $\Delta^{+}$. This implies the equivalence for $X_{-}$, the equivalence for $X_{+}$ is obtained analogously with $\mathcal{U}_{-} / I$.

Theorem 38 Any typical module $V$ over $\mathcal{U}^{H}$ or $\mathcal{U}$ is ambidextrous.

Proof Let $v_{+}, v_{+}^{\prime}$ be (the unique up to a scalar) highest weight vectors of $V, V^{*}$, respectively. Choose lowest weight vectors $v_{-}, v_{-}^{\prime}$ of $V, V^{*}$ so that $d_{V}\left(v_{+}^{\prime} \otimes v_{-}\right)=$ $d_{V}^{\prime}\left(v_{+} \otimes v_{-}^{\prime}\right)=1$ (ie choose scalars of the vectors). We want to apply Theorem 36 where $w_{0}=v_{+} \otimes v_{+}^{\prime}, w_{0}^{\prime}=v_{-} \otimes v_{-}^{\prime}$ and $x, y$ are any elements of $\mathcal{U}$ proportional 
to $\prod_{\alpha \in \gamma_{*}} X_{-\alpha}^{r-1}, \prod_{\alpha \in \gamma_{*}} X_{\alpha}^{r-1}$, respectively, for any convex ordering $\gamma_{*}$ of $\Delta^{+}$and corresponding convex set $\left\{X_{ \pm \gamma}\right\}_{\gamma \in \gamma^{*}}$. First, we will prove that $x . w_{0}$ is a nonzero invariant vector, the proof for $y \cdot w_{0}^{\prime}$ is analogous.

Note that $x \cdot v_{+} \in \mathbb{C}^{*} v_{-}$and the weight of $v_{+}^{\prime}$ is the opposite of the weight of $v_{-}$. Hence, the weight of $w_{0}$ is $2(r-1) \rho$ and weight of $x . w_{0}$ is 0 . We write $x . w_{0}=\sum v_{i} \otimes v_{i}^{\prime}$, where $v_{i}, v_{i}^{\prime}$ are weight vectors. The term of $x . w_{0}$ for which $v_{i}$ has smallest weight is equal to $(x \otimes 1) \cdot\left(v_{+} \otimes v_{+}^{\prime}\right)=\left(x . v_{+}\right) \otimes v_{+}^{\prime} \neq 0$. Moreover, if $j \neq i$ then the weight of $v_{j}$ is strictly larger than the weight of $v_{i}$. Thus, $x . w_{0} \neq 0$.

Next we show $x . w_{0}$ is invariant. It suffices to show that for any $i=1, \ldots, n$ we have $X_{i} \cdot\left(x \cdot w_{0}\right)=0, X_{-i} .\left(x \cdot w_{0}\right)=0$ and $H_{i} \cdot\left(x \cdot w_{0}\right)=0$. First, the last equality is true because as mentioned above $x . w_{0}$ has weight 0 . Second, applying Lemma 37 with a convex ordering $\gamma_{*}$ of $\Delta^{+}$such that $\gamma_{1}=\alpha_{i}$, we can write $x \equiv X_{-i}^{r-1} x^{\prime} \bmod I$ for some $x^{\prime} \in \mathcal{U}_{-}$(note that by definition $X_{\gamma_{1}}=X_{\alpha_{i}}=X_{i}$ ). Then $X_{-i} \cdot\left(x . w_{0}\right)=0$ because $X_{-i} x \equiv X_{-i}^{r} x^{\prime} \equiv 0 \bmod I$. Finally, notice that the weights of the vectors of $\mathcal{U} . w_{0}=\mathcal{U}_{-} . w_{0}$ are of the form $\sum_{j=1}^{N} a_{j} \gamma_{j}$ for $0 \leq a_{j}<r$. But $X_{\alpha_{i}} \cdot\left(x^{\prime} \cdot w_{0}\right)$ has weight $n \alpha_{i}$ so $X_{i} \cdot\left(x^{\prime} \cdot w_{0}\right)=0$. Combining this with the fact that $\left[X_{i}, X_{-i}^{r-1}\right]=$ $[r-1]_{q_{i}}\left[r-2+H_{i}\right]_{q_{i}} X_{-i}^{r-2}$, we have

$$
X_{i} \cdot\left(x . w_{0}\right)=[r-1]_{q_{i}}\left[r-2+H_{i}\right]_{q_{i}} X_{-i}^{r-2} x^{\prime} \cdot w_{0}=[r-1]_{q_{i}}[r]_{q_{i}} X_{-i}^{r-2} x^{\prime} \cdot w_{0}=0
$$

since $H_{i}$ acts on $X_{-i}^{r-2} x^{\prime} . w_{0}$ as 2 and $[r]_{q_{i}}=0$. This proves that $x . w_{0}$ is invariant.

Now we can apply Theorem 36. Indeed, if $V$ is an $\mathcal{U}^{H}$-module, then Condition (a) is satisfied: $w_{0}$ and $w_{0}^{\prime}$ are fixed up to a scalar by any endomorphism of $V \otimes V^{*}$ because they are weight vectors in a one-dimensional weight space. If $V$ is a $\mathcal{U}$-module we can write $V \simeq \mathcal{F}(W)$ for some $W \in O b\left(\mathcal{D}^{H}\right)$. The vector space $W \otimes W^{*}=V \otimes V^{*}$ is equipped with a bilinear form and the weight decomposition of $W \otimes W^{*}$ gives an orthogonal decomposition: $V \otimes V^{*}=\left(\mathbb{C} w_{0} \oplus \mathbb{C} w_{0}^{\prime}\right) \oplus V^{\prime}$ for some $V^{\prime}$ with $\left\langle V^{\prime}, w_{0}\right\rangle=\left\langle V^{\prime}, w_{0}\right\rangle=\left\langle w_{0}, V^{\prime}\right\rangle=\left\langle w_{0}^{\prime}, V^{\prime}\right\rangle=0$. Now the weights in $W \otimes W^{*}$ imply that $\operatorname{ker} x \subset V^{\prime} \oplus \mathbb{C} w_{0}^{\prime}=\operatorname{ker}\left\langle\cdot, w_{0}^{\prime}\right\rangle$ and $\operatorname{ker} y \subset V^{\prime} \oplus \mathbb{C} w_{0}=\operatorname{ker}\left\langle w_{0}, \cdot\right\rangle$. Hence Condition (b) of Theorem 36 is satisfied.

\subsection{The braiding}

We recall some well-known facts about the $h$-adic version of the quantum group $U_{h}(\mathfrak{g})$. The algebra $U_{h}(\mathfrak{g})$ is a $\mathbb{C} \llbracket h \rrbracket$ topological Hopf algebra with generators $X_{i}, X_{-i}, H_{i}$ for $i=1, \ldots, n$ the relation (35), (36) and (37) where $q$ is replaced by $\mathrm{e}^{h / 2}$ and $K_{\alpha_{i}}=q_{i}^{H_{i}}$. For a root $\beta \in L_{R}$, let $q_{\beta}=q^{\langle\beta, \beta\rangle / 2}$. Let $\exp _{q}(x)=\sum_{i=0}^{\infty} x^{i} /[i ; q]$ !, 
where $[i ; q]=\left(1-q^{i}\right) /(1-q)$ and $[i ; q] !=[i ; q] \cdots[1 ; q]$. Consider the following elements of $U_{h}(\mathfrak{g})$ :

$$
H H^{h}=q^{\sum_{i, j} d_{i}\left(A^{-1}\right)_{i j} H_{i} \otimes H_{j}}, \quad \check{R}^{h}=\prod_{\beta \in \beta_{*}} \exp _{q_{\beta}^{-2}}\left(\left(q_{\beta}-q_{\beta}^{-1}\right) X_{\beta} \otimes X_{-\beta}\right),
$$

where the product is ordered by the convex order $\beta_{*}$ of $\Delta^{+}$. It is well known that $R^{h}=H H^{h} \check{R}^{h}$ defines a quasitriangular structure on $U_{h}(\mathfrak{g})$ (see for example [22]). This mean that $R^{h}$ is invertible and satisfies

$$
\Delta \otimes \operatorname{Id}\left(R^{h}\right)=R_{13}^{h} R_{23}^{h}, \quad \operatorname{Id} \otimes \Delta\left(R^{h}\right)=R_{13}^{h} R_{12}^{h}, \quad R^{h} \Delta^{o p}(x)=\Delta(x) R^{h}
$$

for all $x \in U_{h}(\mathfrak{g})$. The algebra $U_{h}(\mathfrak{g})$ admit a PBW basis. Using this basis we can write $U_{h}(\mathfrak{g})$ as a direct sum decomposition: $U_{h}(\mathfrak{g})=U^{<} \oplus I$, where $U^{<}$is the $\mathbb{C} \llbracket h \rrbracket$-module generated by the monomials

$$
\prod_{i=1}^{n} H_{i}^{k_{i}} \prod_{\beta_{i} \in \beta_{*}} X_{\beta_{i}}^{l_{i}} \prod_{\beta_{i} \in \beta_{*}} X_{-\beta_{i}}^{m_{i}} \quad \text { for } 0 \leq l_{i}, m_{i}<r
$$

and $I$ is generated by the other monomial. ${ }^{1}$ Let $p: U_{h}(\mathfrak{g}) \rightarrow U^{<}$be the projection map with kernel $I$. We define

$$
R^{<}=p \otimes p\left(R^{h}\right)=p \otimes \operatorname{Id}\left(R^{h}\right)=\operatorname{Id} \otimes p\left(R^{h}\right) .
$$

Proposition $39 R^{<}$satisfies:

(i) $(p \otimes p \otimes p)\left(\Delta \otimes \operatorname{Id}\left(R^{<}\right)\right)=(p \otimes p \otimes p)\left(R_{13}^{<} R_{23}^{<}\right)$.

(ii) $(p \otimes p \otimes p)\left(\operatorname{Id} \otimes \Delta\left(R^{<}\right)\right)=(p \otimes p \otimes p)\left(R_{13}^{<} R_{12}^{<}\right)$.

(iii) $(p \otimes p)\left(R^{<} \Delta^{o p}(x)\right)=(p \otimes p)\left(\Delta(x) R^{<}\right)$for all $x \in U_{h}(\mathfrak{g})$.

Proof These equalities are obtained by projecting the equalities in (41). Indeed, using $p \circ p=p$ and Equation (42) we have

$$
\begin{aligned}
(p \otimes p \otimes p)\left(\Delta \otimes \operatorname{Id}\left(R^{h}\right)\right) & \left.=(p \otimes p \otimes p) \circ(\Delta \otimes \operatorname{Id}) \circ \operatorname{Id} \otimes p\left(R^{h}\right)\right) \\
& =(p \otimes p \otimes p)\left(\Delta \otimes \operatorname{Id}\left(R^{<}\right)\right) .
\end{aligned}
$$

On the other hand,

$$
\begin{aligned}
(p \otimes p \otimes p)\left(R_{13}^{h} R_{23}^{h}\right) & =(p \otimes p \otimes p)\left((p \otimes \operatorname{Id} \otimes \operatorname{Id})\left(R_{13}^{h}\right)(\operatorname{Id} \otimes p \otimes \operatorname{Id})\left(R_{23}^{h}\right)\right) \\
& =(p \otimes p \otimes p)\left(R_{13}^{<} R_{23}^{<}\right) .
\end{aligned}
$$

This proves the first equality and the proof of the second one is similar.

\footnotetext{
${ }^{1}$ The set $I$ is not an ideal when $q$ is not an $r^{\text {th }}$ root of unity and $U^{<}$depends on $\left(X_{\beta_{*}}\right)$.
} 
To prove the third equality, it suffices to assume $x$ is a generator of the algebra $U_{h}(\mathfrak{g})$. The equality is clear for $x=H_{i}$ as $\Delta\left(H_{i}\right)$ is symmetric and commutes with any of the products $X_{\alpha} \otimes X_{-\alpha}$. Now for $x=X_{i}$, we have $\Delta\left(X_{i}\right)=1 \otimes X_{i}+X_{i} \otimes K_{\alpha_{i}}$. Hence, $(p \otimes p)\left(R^{h}\left(K_{\alpha_{i}} \otimes X_{i}+X_{i} \otimes 1\right)\right)$

$$
\begin{aligned}
& =(p \otimes p)\left((p \otimes \mathrm{Id})\left(R^{h} K_{\alpha_{i}} \otimes X_{i}\right)+(\operatorname{Id} \otimes p)\left(R^{h} X_{i} \otimes 1\right)\right) \\
& =(p \otimes p)\left(R^{<}\left(K_{\alpha_{i}} \otimes X_{i}+X_{i} \otimes 1\right)\right) .
\end{aligned}
$$

On the other hand,

$$
\begin{aligned}
(p \otimes p)\left(\left(1 \otimes X_{i}+X_{i} \otimes\right.\right. & \left.\left.K_{\alpha_{i}}\right) R^{h}\right) \\
& =(p \otimes p)\left((p \otimes \mathrm{Id})\left(1 \otimes X_{i} R^{h}\right)+(\operatorname{Id} \otimes p)\left(X_{i} \otimes K_{\alpha_{i}} R^{h}\right)\right) \\
& =(p \otimes p)\left(\left(1 \otimes X_{i}+X_{i} \otimes K_{\alpha_{i}}\right) R^{<}\right) .
\end{aligned}
$$

The proof is similar for $x=X_{-i}$.

Let $V=V_{1} \otimes \cdots \otimes V_{k}$, where $V_{i} \in O b\left(\mathcal{D}^{H}\right)$ for $i=1, \ldots, k$. Then each $x \in\left(\mathcal{U}^{H}\right)^{\otimes k}$ defines a linear map $\psi(x): V \rightarrow V$ and we have a finite-dimensional representation $\psi:\left(\mathcal{U}^{H}\right)^{\otimes k} \rightarrow \operatorname{End}_{\mathbb{C}}(V)$. For any $x \in\left(\mathcal{U}^{H}\right)^{\otimes k}$, let $\psi\left(q^{x}\right)$ be the limit as $m$ goes to infinity of the absolutely convergent series $\sum_{j=0}^{m} \frac{1}{j !} \psi(2 i \pi x / r)^{j}$. In this situation, we say that $q^{x}$ is an operator on $\mathcal{D}^{H}$. If $q^{x}, q^{y}$ are two operators such that $\psi\left(q^{x}\right)=\psi\left(q^{y}\right)$ for all finite-dimensional representations $\psi$ then we say the operators are equal and write $q^{x}=q^{y}$. One can multiply operators and take their coproduct and antipode using the rules $\Delta q^{x}=q^{\Delta x}$ and $S\left(q^{x}\right)=q^{S(x)}$. We will also call the linear map $\psi(x): V \rightarrow V$ an operator and denote it by $x$.

Let $\mathcal{H}$ be the operator on $\mathcal{D}^{H}$ defined as

$$
\mathcal{H}=q^{\sum_{i, j} d_{i}\left(A^{-1}\right)_{i j} H_{i} \otimes H_{j}} .
$$

Lemma 40 For any $x, y \in \mathcal{U}^{H}$ with weights $\alpha, \beta \in L_{W}$, respectively, we have the equalities of operators on $\mathcal{D}^{H}$ :

$$
\mathcal{H}(x \otimes y)=q^{\langle\alpha, \beta\rangle}\left(x K_{\beta} \otimes y K_{\alpha}\right) \mathcal{H} .
$$

Also, $\Delta \otimes \operatorname{Id}(\mathcal{H})=\mathcal{H}_{13} \mathcal{H}_{23}$ and $\operatorname{Id} \otimes \Delta(\mathcal{H})=\mathcal{H}_{13} \mathcal{H}_{12}$.

Proof The proof is a direct computation using the following three facts: (1) $H_{i} x=$ $x\left(H_{i}+\frac{1}{d_{i}}\left\langle\alpha_{i}, \alpha\right\rangle\right),(2)$ as operators $K_{\alpha_{i}}=q_{i}^{H_{i}}$ and (3)

$$
\mathcal{H}\left(v_{\lambda} \otimes w_{\mu}\right)=q^{\langle\lambda, \mu\rangle} v_{\lambda} \otimes w_{\mu}
$$

for any weight vectors $v_{\lambda}$ and $w_{\mu}$ of weight $\lambda$ and $\mu$, respectively. 
Let $\check{R}$ be the truncated quasi $R$-matrix of $\mathcal{U}^{H}$ given by

$$
\check{R}=\prod_{i=1}^{N}\left(\sum_{j=0}^{r-1} \frac{\left(\left(q_{\beta_{i}}-q_{\beta_{i}}^{-1}\right) X_{\beta_{i}} \otimes X_{-\beta_{i}}\right)^{j}}{\left[j ; q_{\beta_{i}}^{-2}\right] !}\right) \in \mathcal{U}_{+} \otimes \mathcal{U}_{-},
$$

where $\beta_{*}=\left(\beta_{1}, \ldots, \beta_{N}\right)$ is a convex set of $\Delta^{+}$.

Theorem 41 The operator $\mathcal{R}=\mathcal{H} \check{R}$ leads to a braiding $\left\{c_{V, W}\right\}$ in $\mathcal{D}^{H}$, where $c_{V, W}: V \otimes W \rightarrow W \otimes V$ is given by $v \otimes w \mapsto \tau(\mathcal{R}(v \otimes w))$. Here $\tau: V \otimes W \rightarrow W \otimes V$ is the trivial isomorphism of vector spaces given by permutation.

Proof It is enough to show that the operator $\mathcal{R}$ satisfies

$$
\Delta \otimes \operatorname{Id}(\mathcal{R})=\mathcal{R}_{13} \mathcal{R}_{23}, \quad \operatorname{Id} \otimes \Delta(\mathcal{R})=\mathcal{R}_{13} \mathcal{R}_{12}, \quad \mathcal{R} \Delta^{o p}(x)=\Delta(x) \mathcal{R}
$$

for all $x \in \mathcal{U}^{H}$. Let $\chi: U_{h}(\mathfrak{g}) \otimes U_{h}(\mathfrak{g}) \rightarrow U_{h}(\mathfrak{g}) \otimes U_{h}(\mathfrak{g})$ be the map given by $x \otimes y \mapsto H H^{h}(x \otimes y)\left(H H^{h}\right)^{-1}$. Viewing $\check{R}$ as element of $U_{h}(\mathfrak{g})$ we have that Proposition 39 induces the relations

$$
\begin{aligned}
\Delta \otimes \operatorname{Id}(\check{R}) & =\chi_{23}\left(\check{R}_{13}\right) \check{R}_{23}, \\
\operatorname{Id} \otimes \Delta(\check{R}) & =\chi_{12}\left(\check{R}_{13}\right) \check{R}_{12}, \\
\check{R} \Delta^{o p}(x) & =\chi(\Delta(x)) \check{R},
\end{aligned}
$$

for all $x \in U_{h}(\mathfrak{g})$. Let us prove the first equality in (46); the other two are similar. The first equation of Proposition 39 implies that $\Delta \otimes \operatorname{Id}\left(H H^{h} \check{R}\right)=H H^{h}{ }_{13} \check{R}_{13} H H^{h}{ }_{23} \check{R}_{23}$. The left-hand side of this equality is equal to

$$
\Delta \otimes \operatorname{Id}\left(H H^{h}\right) \Delta \otimes \operatorname{Id}(\check{R})=H H^{h}{ }_{13} H H^{h}{ }_{23} \Delta \otimes \operatorname{Id}(\check{R}) .
$$

On the other hand,

$$
H H^{h}{ }_{13} \check{R}_{13} H H^{h}{ }_{23} \check{R}_{23}=H H^{h}{ }_{13} H H^{h}{ }_{23} \chi_{23}\left(\check{R}_{13}\right) \check{R}_{23} .
$$

Now since $H H^{h}$ is invertible we have the desired result.

The element $R^{<}$does not have a pole at $q$ when $q$ is a primitive root of unity of order $r$. Thus, the relations of Equation (46) hold when $q$ is a root of unity. Finally, Lemma 40 implies that the operators $H H^{h}$ and $\mathcal{H}$ satisfy the same commutator relations on $U_{h}(\mathfrak{g})$ and $\mathcal{U}^{H}$, respectively. Thus, the relations of Equation (45) hold.

Consider the operator $u_{\mathcal{H}}=q^{\sum_{i, j}-d_{i}\left(A^{-1}\right)_{i j} H_{i} H_{j}}$. Then $S\left(u_{\mathcal{H}}\right)=u_{\mathcal{H}}$ and if $v$ is a weight vector in a $\mathcal{U}^{H}$-module of weight $\lambda$ then $u_{\mathcal{H}} v=q^{-\langle\lambda, \lambda\rangle} v$. Also, if $x \in \mathcal{U}^{H}$ has weight $\alpha$, then $u_{\mathcal{H}} x=q^{-\langle\alpha, \alpha\rangle} x K_{-\alpha}^{2} u_{\mathcal{H}}$ and conjugation by $u_{\mathcal{H}}{ }^{-1}$ induces a well 
defined automorphism $\operatorname{Ad}_{u_{\mathcal{H}}-1}$ of $\mathcal{U}_{\mathfrak{h}}$. Let $u=\mu \circ\left(\left(\operatorname{Ad}_{u_{\mathcal{H}}-1} \circ S\right) \otimes \operatorname{Id}\right)\left(\check{R}_{21}\right) \in \mathcal{U}_{-} \mathcal{U}_{+}$, where $\mu$ is the multiplication. In particular, since $u$ has weight 0 , the operators $u_{\mathcal{H}}$ and $u$ commute.

Let $v$ and $\theta$ be the operators $v=u_{\mathcal{H}} u$ and $\theta=v K_{2 \rho}^{r-1}$. Then

$$
\theta S(\theta)^{-1}=v K_{2 \rho}^{r-1} S(v)^{-1} K_{2 \rho}^{r-1}=u S(u)^{-1} K_{2 \rho}^{2(r-1)} .
$$

We define $\varpi=u S(u)^{-1} K_{2 \rho}^{2(r-1)} \in \mathcal{U}^{H}$.

Proposition 42 Let $V_{\lambda}$ be a typical $\mathcal{U}^{H}$-module then $\varpi: V_{\lambda} \rightarrow V_{\lambda}$ is the identity.

Proof Let $v_{+}$and $v_{-}$be the highest and lowest weight vectors of $V_{\lambda}$ of weights $\lambda$ and $\lambda-2(r-1) \rho$, respectively. Then $u-1 \in \mathcal{U}_{0} \mathcal{U}_{-}-\mathcal{U}_{++}$implies that $u$ acts by 1 on $v_{+}$. Similarly, $S(u)$ acts by 1 on $v_{-}$. In particular, $\theta\left(v_{+}\right)=q^{-\langle\lambda, \lambda\rangle} q^{\langle 2 \rho(r-1), \lambda\rangle} v_{+}$ and

$$
\begin{aligned}
(S(\theta))\left(v_{-}\right) & =q^{-\langle 2(r-1) \rho, \lambda-2(r-1) \rho\rangle} q^{-\langle\lambda-2(r-1) \rho, \lambda-2(r-1) \rho\rangle} v_{-} \\
& =q^{-\langle\lambda, \lambda\rangle} q^{\langle 2 \rho(r-1), \lambda\rangle} v_{-} .
\end{aligned}
$$

But $\theta$ and $S(\theta)$ are central and act as scalars on $V_{\lambda}$. Thus,

$$
\theta_{\mid V_{\lambda}}=S(\theta)_{\mid V_{\lambda}}=q^{-\langle\lambda, \lambda-2 \rho(r-1)\rangle} \mathrm{Id} .
$$

Theorem 43 Let $\mathcal{D}^{\theta}$ be the full subcategory of $\mathcal{D}^{H}$ formed by modules on which $\varpi$ acts as the identity. Then $\mathcal{D}^{\theta}$ is a $\mathbb{C}$-linear ribbon category with braiding

$$
c_{V, W}: V \otimes W \rightarrow W \otimes V, \quad v \otimes w \mapsto \tau(\mathcal{R}(v \otimes w))
$$

and twist

$$
\theta_{V}: V \rightarrow V, \quad v \mapsto \theta^{-1}(v)
$$

Proof Recall that $I^{H}$ is the ideal of $\mathcal{U}^{H}$ generated by the central elements $X_{ \pm \beta}^{r}$, where $\beta \in \beta_{*}$. Remark that $\varpi$ is central and group-like in the quotient Hopf algebra $\mathcal{U}^{H} / I^{H}$. Hence $\mathcal{D}^{\theta}$ is a tensor subcategory of $\mathcal{D}^{H}$. The braiding of $\mathcal{D}^{H}$ given Theorem 41 restricts to a braiding on $\mathcal{D}^{\theta}$. Finally, since $\varpi$ acts as the identity on any object in $\mathcal{D}^{\theta}$ we have that operators $\theta$ and $S(\theta)$ are equal. It follows that $\theta_{V}$ is a twist on $\mathcal{D}^{\theta}$.

Remark that from Proposition 42 the category $\mathcal{D}^{\theta}$ contains all the typical modules of $\mathcal{D}^{H}$.

Conjecture $44 \varpi=1$ and $\mathcal{D}^{H}=\mathcal{D}^{\theta}$ is a ribbon category. 


\subsection{The modified dimension}

As irreducible projective modules of $\mathcal{D}$ and $\mathcal{D}^{H}$ are ambidextrous, there is an unique nontrivial trace on the ideal of projective objects (cf [18]). On simple objects, the trace is given by the modified dimension which we calculate for the special case of nilpotent modules. Then we show that the modified dimension is the same for an object and its dual, thus leading to a relative $G$-spherical structure in $\mathcal{D}$.

Proposition 45 Let $\lambda, \mu \in \operatorname{Hom}_{\mathrm{alg}}\left(\mathcal{U}_{\mathfrak{h}}, \mathbb{C}\right)$ be additive weights where $V_{\lambda}$ is typical. Then

$$
S^{\prime}(\lambda, \mu)=\left\langle V_{\lambda} \underset{\downarrow}{ } \mid V_{\mu}\right\rangle=q^{2\langle\mu+(1-r) \rho, \lambda\rangle} \prod_{\alpha \in \Delta^{-}} \frac{q^{2 r\langle\mu+(1-r) \rho, \alpha\rangle}-1}{q^{2\langle\mu+(1-r) \rho, \alpha\rangle}-1} \in \mathbb{C},
$$

where $\Delta^{-}=-\Delta^{+}$is the set of negative roots and as above $\langle f\rangle \in \mathbb{C}$ of a morphism $f: V_{\lambda} \rightarrow V_{\lambda}$ is the scalar defined by $f=\langle f\rangle \operatorname{Id}_{V_{\lambda}}$.

Proof Let $\left\{v_{i}\right\}$ be a basis of $V_{\lambda}$ such that $v_{i}$ is a weight vector with additive weight $\lambda_{i}$. Let $w_{\mu}$ be a highest weight vector of $V_{\mu}$. From Equation (44) we have

$$
\mathcal{H}\left(w_{\mu} \otimes v_{i}\right)=q^{\left\langle\mu, \lambda_{i}\right\rangle} w_{\mu} \otimes v_{i} \quad \text { and } \quad \mathcal{H}\left(v_{i} \otimes w_{\mu}\right)=q^{\left\langle\mu, \lambda_{i}\right\rangle} v_{i} \otimes w_{\mu} .
$$

We now give two facts. Let $v$ be any weight vector of $V_{\lambda}$ of weight $\eta$.

Fact $1 \mathcal{R}\left(w_{\mu} \otimes v\right)=q^{\langle\mu, \eta\rangle}\left(w_{\mu} \otimes v\right)$.

This fact follows from the definition of $\check{R}$, Equation (48) and the property that $E_{\alpha} w_{\mu}=$ 0 for $\alpha \in \Delta^{+}$.

Fact 2 All the pure tensors of the element $(\check{R}-1)\left(v \otimes v_{\mu}\right) \in V_{\lambda} \otimes V_{\mu}$ are of the form $v^{\prime} \otimes w^{\prime}$, where $w^{\prime}$ is a weight vector of $V_{\mu}$ and $v^{\prime}$ is a weight vector of $V_{\lambda}$ whose weight is of strictly higher order than that of the weight of $v$.

Fact 2 is true because $E_{\alpha}^{n} v$ (for $\alpha \in \Delta^{+}$and $n \in \mathbb{N}_{>0}$ ) is zero or a weight vector whose weight is of strictly higher order than the weight of $v$.

We will now compute $S^{\prime}(\lambda, \mu)$ directly. Let $V$ be a typical module and recall that the duality morphisms $d_{V}^{\prime}: V \otimes V^{*} \rightarrow \mathbb{C}$ is defined as

$$
d_{V_{\lambda}}^{\prime}(v \otimes f)=f\left(q^{2(1-r)\langle\eta, \rho\rangle} v\right),
$$


where $v$ is a weight vector of $V_{\lambda}$ of additive weight $\eta$. Consider the element $S$ of $\operatorname{End}_{\mathbb{C}}\left(V_{\mu}\right)$ given by

$$
\left(\operatorname{Id}_{V_{\mu}} \otimes d_{V_{\lambda}}^{\prime}\right) \circ\left(c_{V_{\lambda}, V_{\mu}} \otimes \operatorname{Id}_{V_{\lambda}^{*}}\right) \circ\left(c_{V_{\mu}, V_{\lambda}} \otimes \operatorname{Id}_{V_{\lambda}^{*}}\right) \circ\left(\operatorname{Id}_{V_{\mu}} \otimes b_{V_{\lambda}}\right) .
$$

To simplify notation set $S=\left(X_{1}\right)\left(X_{2}\right)\left(X_{3}\right)\left(X_{4}\right)$, where $X_{i}$ is the corresponding morphism in the above formula. The morphism $S$ is determined by its value on the highest weight $w_{\mu}$. By definition $S\left(w_{\mu}\right)=S^{\prime}(\lambda, \mu) w_{\mu}$, so it suffices to compute $S\left(w_{\mu}\right)$ :

$$
\begin{aligned}
S\left(w_{\mu}\right) & =\left(X_{1}\right)\left(X_{2}\right)\left(X_{3}\right)\left(w_{\mu} \otimes \sum_{i}\left(v_{i} \otimes v_{i}^{*}\right)\right) \\
& =\left(X_{1}\right)\left(X_{2}\right)\left(\sum_{i} q^{\left\langle\mu, \lambda_{i}\right\rangle} v_{i} \otimes w_{\mu} \otimes v_{i}^{*}\right) \\
& =\left(X_{1}\right)\left(\sum_{i}\left(q^{2\left\langle\mu, \lambda_{i}\right\rangle} w_{\mu} \otimes v_{i} \otimes v_{i}^{*}\right)+\sum_{k} w_{k}^{\prime} \otimes v_{k}^{\prime} \otimes z_{k}\right) \\
& =\sum_{i} q^{2\left\langle\mu+(1-r) \rho, \lambda_{i}\right\rangle} w_{\mu},
\end{aligned}
$$

where $z_{k}=v_{i}^{*}$ (for some $i$ ), $v_{k}^{\prime}$ is a weight vector of $V_{\lambda}$ whose weight is of strictly higher weight than that of the weight of $z_{k}^{*}$ and $w_{k}^{\prime}$ is a weight vector of $V_{\mu}$. Moreover, the second equality of the above equation follows from Fact 1, the third from (48) and Fact 2 and finally the fourth from Equation (49) and the fact that $z_{k}\left(v_{k}^{\prime}\right)=0$. The key observation in this proof is that Facts 1 and 2 imply that in the above computation the only contribution of the action of the operator $\mathcal{R}$ comes from $\mathcal{H}$.

Finally, $V_{\lambda}$ is typical and so its weights are determined the formal sum (ie its character):

$$
\sum_{i} \mathrm{e}^{\lambda_{i}}=\mathrm{e}^{\lambda} \prod_{\alpha \in \Delta^{-}} \frac{\mathrm{e}^{r \alpha}-1}{\mathrm{e}^{\alpha}-1} .
$$

Thus, the proposition follows from Equation (50).

We now can apply the results of Section 2 to $\mathcal{D}^{\theta}$. From Theorem 38 we know that any typical $\mathcal{U}^{H}$-module $J$ is ambidextrous. Therefore, we can apply Theorem 8 to $\mathcal{D}^{\theta}$ and obtain an ambi pair $\left(A_{\text {Proj }}, d\right)$. As discussed in Section 2 the functions $d$ and $d_{J}$ are proportional. Now, let $\lambda \in \operatorname{Hom}_{\mathrm{alg}}\left(\mathcal{U}_{\mathfrak{h}}, \mathbb{C}\right)$ be an additive weight. Combining the formula in Proposition 45 with the formula

$$
\prod_{\alpha \in \Delta^{-}}\left(q^{2\langle\lambda+(1-r) \rho, \alpha\rangle}-1\right)=q^{\langle\lambda+(1-r) \rho,-2 \rho\rangle} \prod_{\alpha \in \Delta^{+}}\{\langle\lambda+(1-r) \rho, \alpha\rangle\}
$$


we can see that the function $d$ can be multiplied by a scalar in $\mathbb{C}^{\times}$so that it is given by the formula

$$
\mathrm{d}\left(V_{\lambda}\right)=\prod_{\alpha \in \Delta^{+}} \frac{\{\langle\lambda-(r-1) \rho, \alpha\rangle\}}{\{r\langle\lambda-(r-1) \rho, \alpha\rangle\}}
$$

for $V_{\lambda}$ with $|\lambda| \notin \mathcal{X}$. Thus we have proved:

Theorem 46 The category $\mathcal{D}^{\theta}$ is a ribbon category with a projective ambidextrous object and so gives rise to an ambi pair $\left(A_{\text {Proj }}, d\right)$ and isotopy invariant $\mathrm{G}^{\prime}$, where $\mathrm{d}$ satisfies Equation (51).

It is clear from (51) that $\mathrm{d}\left(V_{\lambda}\right)$ depends only on the multiplicative weight $\kappa=q^{\lambda} \in$ Hom $_{\text {alg }}\left(\mathcal{U}_{0}, \mathbb{C}\right)$ induced by $\lambda$. Thus we can define $\mathrm{d}\left(V_{\kappa}\right)=\mathrm{d}\left(V_{\lambda}\right)$ whenever $|\kappa| \notin \mathcal{X}$. Let $\mathrm{A}$ be the set of irreducible $\mathcal{U}$-modules $V$ such that $V \in \mathcal{D}_{g}$ for some $g \in \mathcal{G} \backslash \mathcal{X}$. Theorem 32 implies that each module in A is projective. Let Proj be the full subcategory of $\mathcal{D}$ consisting of projective $\mathcal{U}$-modules. Recall the notion of a trace given in Section 1.2.

Lemma 47 There exist a trace $\mathrm{t}=\left\{\mathrm{t}_{V}\right\}$ on Proj such that $\mathrm{t}_{V_{\kappa}}\left(\operatorname{Id}_{V_{\kappa}}\right)=\mathrm{d}\left(V_{\kappa}\right)$ for any $V_{\kappa}$ of multiplicative weight $\kappa=q^{\lambda}$ with $|\lambda| \notin \mathcal{X}$.

Proof Let $U \in \mathcal{D}_{g}$ be a typical projective $\mathcal{U}$-module with $g \notin \mathcal{X}$. Consider the linear map $t: \operatorname{End}_{\mathcal{C}}(U) \rightarrow \mathbb{C}$ given by $f \mapsto \mathrm{d}(U)\langle f\rangle$ where, as above, $\langle f\rangle$ is defined by $f=\langle f\rangle \operatorname{Id}_{U}$. From Theorem 38 we have that $U$ is ambidextrous. Then from [18] we have that $t$ determines a unique trace $\mathrm{t}=\left\{\mathrm{t}_{V}\right\}$ on Proj such that $t=\mathrm{t}_{V}$.

On the other hand, $\mathrm{F}^{\prime}$ defines a trace $\mathrm{t}^{\prime}=\left\{\mathrm{t}_{V}^{\prime}\right\}$ on the projective modules of $\mathcal{D}^{H}$ given by $\mathrm{t}_{V}^{\prime}(f)=\mathrm{F}^{\prime}\left(L_{f}\right)$, where $L_{f}$ is ribbon graph which is the closure of coupon of $f$. Then by definition, for any additive weight $\lambda$ with $|\lambda| \notin \mathcal{X}$, we have $\mathrm{t}_{V_{\lambda}}^{\prime}\left(\operatorname{Id}_{V_{\lambda}}\right)=$ $\mathrm{F}^{\prime}\left(O_{V_{\lambda}}\right)=\mathrm{d}\left(V_{\lambda}\right)$, where $O_{V_{\lambda}}$ is the unknot colored with $V_{\lambda}$.

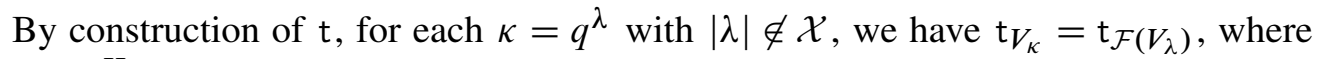
$\mathcal{F}: \mathcal{D}^{H} \rightarrow \mathcal{D}$ is the forgetful functor defined above. Moreover, $\mathrm{t}_{\mathcal{F}\left(V_{\lambda}\right)}(\mathcal{F}(f))=\mathrm{t}_{V_{\lambda}}^{\prime}(f)$ for all $f \in \operatorname{End}\left(V_{\lambda}\right)$. Thus, $\mathrm{t}_{V_{\kappa}}\left(\operatorname{Id}_{V_{\kappa}}\right)=\mathrm{t}_{V_{\lambda}}^{\prime}\left(\operatorname{Id}_{V_{\lambda}}\right)=\mathrm{d}\left(V_{\lambda}\right)=\mathrm{d}\left(V_{\kappa}\right)$.

Lemma 47 implies the assignment $d$ on nilpotent $\mathcal{U}$-modules can be extended to a function $\mathrm{d}: \mathrm{A} \rightarrow \mathbb{C}$ given by $\mathrm{d}(V)=\mathrm{t}_{V}\left(\mathrm{Id}_{V}\right)$. Since $V \in \mathrm{A}$ is projective it follows from [18] that $\mathrm{d}(V) \neq 0$. Let $s: \mathrm{A} \rightarrow \mathbb{C}$ be the slope given by $s(V)=\mathrm{d}(V) / \mathrm{d}\left(V^{*}\right)$. It satisfies

$$
s(W)=s(U) s(V)
$$

whenever $W$ is a direct summand of $U \otimes V$ (see [18]). 
Lemma 48 For any $V \in \mathrm{A}$, we have $s(V)=1$ and $\operatorname{so} \mathrm{d}(V)=\mathrm{d}\left(V^{*}\right)$.

Proof First, we will show that for an irreducible $V \in \mathcal{D}_{g}$ the slope $s(V)$ depends only of the degree $g \in \mathcal{G}$. In [11] it is shown that for any $g, g^{\prime}, g g^{\prime} \in \mathcal{G} \backslash \mathcal{X}$ and any irreducible modules $V \in \mathcal{D}_{g}, V^{\prime} \in \mathcal{D}_{g^{\prime}}$ we have

$$
V \otimes V^{\prime} \simeq \bigoplus_{W \in \operatorname{irr}\left(\mathcal{D}_{g g^{\prime}}\right)} W^{\oplus r^{N-n}},
$$

where $\operatorname{irr}\left(\mathcal{D}_{g g^{\prime}}\right)$ denotes a representing set of the isomorphism classes of irreducible modules of $\mathcal{D}_{g g^{\prime}}$. This implies that if $W_{1}, W_{2} \in \mathcal{D}_{g}$ are irreducible modules then there exist projective irreducible modules $U, V$ such that both $W_{1}$ and $W_{2}$ are direct summands of $U \otimes V$. Then by Equation (52) we have $s\left(W_{1}\right)=s\left(W_{2}\right)=s(U) s(V)$. Thus, the slope factor through a map on $\mathcal{G} \backslash \mathcal{X}$ also denoted by $s$ which satisfies $s\left(g g^{\prime}\right)=s(g) s\left(g^{\prime}\right)$ and $s\left(g^{-1}\right)=1 / s(g)$.

Next we show that $s$ extends to a character on the whole group $\mathcal{G}:$ If $x \in \mathcal{X}$, choose $g \in \mathcal{G} \backslash\left(\mathcal{X} \cup \mathcal{X} x^{-1}\right)$ then we can define $s(x)$ as the ratio $s(g x) / s(g)$. This is well defined: if $h \in \mathcal{G} \backslash\left(\mathcal{X} \cup \mathcal{X} x^{-1}\right)$ then there exists $k \in \mathcal{G} \backslash\left(\mathcal{X} \cup \mathcal{X} x^{-1} \cup \mathcal{X} g \cup \mathcal{X} h\right)$ for which $k, k x, k g^{-1}, k h^{-1} \notin \mathcal{X}$ and thus $s(k x) / s(g x)=s\left(k g^{-1}\right)=s(k) / s(g)$, $s(k x) / s(h x)=s\left(k h^{-1}\right)=s(k) / s(h)$ which imply that $s(g x) / s(g)=s(k x) / s(k)=$ $s(h x) / s(h)$.

But now Equation (51) implies that $T \subset \operatorname{ker} s$. As the normal subgroup of the Borel $B_{+} \subset \mathcal{G}$ generated by its Cartan subgroup $T$ is $B_{+}$itself, this implies that $B_{+} \subset \operatorname{ker} s$. Similarly $B_{-} \subset \operatorname{ker} s$ thus $\operatorname{ker} s \supset B_{+} B_{-}=\mathcal{G}$.

Lemma 49 The pair $(A, d)$ is $t$-ambi.

Proof From Lemmas 47 and 48 there exists a trace $\mathrm{t}=\left\{\mathrm{t}_{V}\right\}_{V \in \text { Proj }}$ such that $\mathrm{d}(V)=$ $\mathrm{d}\left(V^{*}\right)$ for all $V \in$ Proj. Then from [18] we have that $\left(\mathrm{A}_{\text {Proj }}, \mathrm{d}\right)$ is t-ambi.

Let us increase $\mathcal{X}$ by adding all $g \in \mathcal{G}$ with $g^{2}=1$. Then $\mathcal{G} \backslash \mathcal{X}$ is still a Zariski open dense subset of $\mathcal{G}$ and by Lemma 3 there exists basic data for $\mathcal{D}$.

Theorem 50 The category $\mathcal{D}$ of finite-dimensional weight modules over $\mathcal{U}$ is a $(\mathcal{X}, \mathrm{d})$-relative $\mathcal{G}$-spherical category which admits basic data.

Proof The category $\mathcal{D}$ is a $\mathbb{C}$-linear pivotal category where the pivotal structure is given in Section 5.6. The $\mathcal{G}$-grading of $\mathcal{D}$ is defined in Section 5.3. Item (i) of Definition 2 follows from Theorem 32. Lemma 49 implies Item (ii). Finally, since the objects of $\mathcal{D}$ are finite-dimensional vector spaces it follows from [17] that the map b: $A \rightarrow \mathbb{C}$ defined in Equation (3) is well defined and satisfies all the properties of Item (iii). 


\section{References}

[1] Y Akutsu, T Deguchi, T Ohtsuki, Invariants of colored links, J. Knot Theory Ramifications 1 (1992) 161-184 MR1164114

[2] S Baseilhac, R Benedetti, Quantum hyperbolic invariants of 3-manifolds with PSL(2, C)-characters, Topology 43 (2004) 1373-1423 MR2081430

[3] S Baseilhac, R Benedetti, Quantum hyperbolic geometry, Algebr. Geom. Topol. 7 (2007) 845-917 MR2336244

[4] J Bichon, Cosovereign Hopf algebras, J. Pure Appl. Algebra 157 (2001) 121-133 MR1812048

[5] R Brown, Groupoids and van Kampen's theorem, Proc. London Math. Soc. 17 (1967) 385-401 MR0220279

[6] V Chari, A Pressley, A guide to quantum groups, Cambridge Univ. Press (1994) MR1300632

[7] F Costantino, N Geer, B Patureau-Mirand, Quantum invariants of 3-manifolds via link surgery presentations and non-semi-simple categories arXiv:1202.3553

[8] C De Concini, V G Kac, Representations of quantum groups at roots of 1, from: "Operator algebras, unitary representations, enveloping algebras, and invariant theory", (A Connes, M Duflo, A Joseph, R Rentschler, editors), Progr. Math. 92, Birkhäuser, Boston, MA (1990) 471-506 MR1103601

[9] C De Concini, V G Kac, C Procesi, Quantum coadjoint action, J. Amer. Math. Soc. 5 (1992) 151-189 MR1124981

[10] C De Concini, V G Kac, C Procesi, Some remarkable degenerations of quantum groups, Comm. Math. Phys. 157 (1993) 405-427 MR1244875

[11] C De Concini, C Procesi, N Reshetikhin, M Rosso, Hopf algebras with trace and representations, Invent. Math. 161 (2005) 1-44 MR2178656

[12] N Geer, R Kashaev, V Turaev, Tetrahedral forms in monoidal categories and 3manifold invariants, J. Reine Angew. Math. 673 (2012) 69-123 MR2999129

[13] N Geer, J Kujawa, B Patureau-Mirand, Generalized trace and modified dimension functions on ribbon categories, Selecta Math. 17 (2011) 453-504 MR2803849

[14] N Geer, B Patureau-Mirand, Multivariable link invariants arising from $\mathfrak{s l}(2 \mid 1)$ and the Alexander polynomial, J. Pure Appl. Algebra 210 (2007) 283-298 MR2311186

[15] N Geer, B Patureau-Mirand, Multivariable link invariants arising from Lie superalgebras of type I, J. Knot Theory Ramifications 19 (2010) 93-115 MR2640994

[16] N Geer, B Patureau-Mirand, V Turaev, Modified quantum dimensions and renormalized link invariants, Compos. Math. 145 (2009) 196-212 MR2480500

[17] N Geer, B Patureau-Mirand, V Turaev, Modified $6 j-$-symbols and 3-manifold invariants, Adv. Math. 228 (2011) 1163-1202 MR2822220 
[18] N Geer, B Patureau-Mirand, A Virelizier, Modified traces and $6 j-$ symbols in pivotal categories, in preparation

[19] A Hatcher, Algebraic topology, Cambridge Univ. Press (2002) MR1867354

[20] R M Kashaev, Quantum dilogarithm as a 6j-symbol, Modern Phys. Lett. A 9 (1994) 3757-3768 MR1317945

[21] R M Kashaev, A link invariant from quantum dilogarithm, Modern Phys. Lett. A 10 (1995) 1409-1418 MR1341338

[22] S Majid, Foundations of quantum group theory, Cambridge Univ. Press (1995) MR1381692

[23] H Murakami, J Murakami, The colored Jones polynomials and the simplicial volume of a knot, Acta Math. 186 (2001) 85-104 MR1828373

[24] W D Neumann, Combinatorics of triangulations and the Chern-Simons invariant for hyperbolic 3-manifolds, from: “Topology '90", (B Apanasov, W D Neumann, A W Reid, L Siebenmann, editors), Ohio State Univ. Math. Res. Inst. Publ. 1, de Gruyter, Berlin (1992) 243-271 MR1184415

[25] W D Neumann, Hilbert's 3 rd problem and invariants of 3-manifolds, from: "The Epstein birthday schrift", (I Rivin, C Rourke, C Series, editors), Geom. Topol. Monogr. 1 (1998) 383-411 MR1668316

[26] J Petit, Decomposition of the Turaev-Viro TQFT arXiv:0903.4512

[27] V G Turaev, Quantum invariants of knots and 3-manifolds, de Gruyter Studies in Mathematics 18, Walter de Gruyter \& Co., Berlin (1994) MR1292673

[28] V Turaev, Homotopy quantum field theory, EMS Tracts in Mathematics 10, Eur. Math. Soc., Zürich (2010) MR2674592

[29] V G Turaev, O Y Viro, State sum invariants of 3-manifolds and quantum $6 j-$-symbols, Topology 31 (1992) 865-902 MR1191386

[30] V Turaev, H Wenzl, Quantum invariants of 3-manifolds associated with classical simple Lie algebras, Internat. J. Math. 4 (1993) 323-358 MR1217386

Mathematics and Statistics, Utah State University

3900 Old Main Hill, Logan, UT 84322-3900, USA

LMAM, Université de Bretagne-Sud

BP 573, 56017 Vannes, France

nathan.geer@usu.edu, bertrand.patureau@univ-ubs.fr

Received: 3 July 2012 Revised: 17 May 2013 